Supplement of Geosci. Model Dev., 10, 1521-1548, 2017

http://www.geosci-model-dev.net/10/1521/2017/

doi:10.5194/gmd-10-1521-2017-supplement

(C) Author(s) 2017. CC Attribution 3.0 License.

(c) (i)

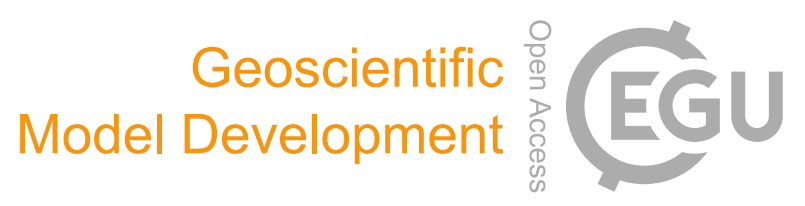

Supplement of

\title{
Collection/aggregation algorithms in Lagrangian cloud microphysical mod- els: rigorous evaluation in box model simulations
}

Simon Unterstrasser et al.

Correspondence to: Simon Unterstrasser (simon.unterstrasser@ dlr.de)

The copyright of individual parts of the supplement might differ from the CC-BY 3.0 licence. 


\section{Contents}

1 Introduction $\quad 5$

2 Simulation Results of Remapping Algorithm (RMA) 6

2.1 Golovin Kernel (RMA) . . . . . . . . . . . . . . . . . . . 7

2.1.1 Golovin Kernel (RMA), regular RMA version . . . . . . . . . . . 8

2.1.1.1 Variation of $d t$ and $\kappa \ldots \ldots . \ldots . \ldots 8$

2.1.1.2 Variation of $\eta \ldots \ldots \ldots$. . . . . . . . . . . . . . . . . . . . . . . . . .

2.1.1.3 Strict threshold, variation of $\eta$. . . . . . . . . . . . 10

2.1.2 Golovin Kernel (RMA), RMA with Reduction Limiter . . . . . . . . 11

2.1.2.1 Variation of $d t$ and $\kappa \ldots \ldots . \ldots . \ldots . \ldots 11$

2.1.2.2 Variation of $r_{\text {critmin }}$ and $\eta \ldots \ldots \ldots . \ldots . \ldots . \ldots 12$

2.1.2.3 Variation of $\tilde{\gamma} \ldots \ldots \ldots 13$

2.1.3 Golovin Kernel (RMA), RMA with Update on the Fly $\mathrm{OTF}_{s}$. . . . . 14

2.1.3.1 Variation of $d t$ and $\kappa \ldots \ldots . \ldots . . . . . . . . .14$

2.1.4 Golovin Kernel (RMA), RMA with Update on the Fly $\mathrm{OTF}_{l} \ldots \ldots$

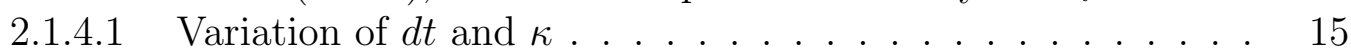

2.2 Long Kernel (RMA) . . . . . . . . . . . . . . . . . . . . . 16

2.2.1 Long Kernel, RMA with Reduction Limiter . . . . . . . . . . . . . 17

2.2.1.1 Variation of $d t$ and $\kappa \ldots \ldots . \ldots . \ldots 17$

2.2.1.2 Variation of $t_{\text {init }}$ and $\eta \ldots \ldots \ldots . \ldots . \ldots 18$

2.2.2 Long Kernel, RMA with Update on the Fly $\mathrm{OTF}_{l}$. . . . . . . . . . . 19

2.2.2.1 Variation of $d t$ and $\kappa \ldots \ldots . \ldots . \ldots . \ldots . . \ldots 19$

3 Simulation Results of Average Impact Algorithm (AIM) 20

3.1 Golovin Kernel (AIM) _ . . . . . . . . . . . . . . . . . . . . . . . 21

3.1 .1 Golovin Kernel (AIM), SingleSIP-init . . . . . . . . . . . . . . 22

3.1.1.1 Variation of $d t$ and $\kappa \ldots \ldots \ldots . \ldots . \ldots . \ldots 22$

3.1 .2 Golovin Kernel (AIM), $\nu_{\text {const }}$-init . . . . . . . . . . . . . . . 23

3.1.2.1 Variation of $N_{S I P} \ldots \ldots \ldots \ldots \ldots \ldots$

3.1.3 Golovin Kernel (AIM), $\nu_{d r a w}$-init . . . . . . . . . . . . . . 24

3.1.3.1 Variation of $N_{S I P} \ldots \ldots \ldots \ldots . \ldots \ldots 24$

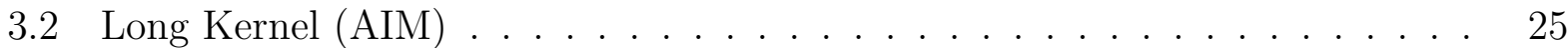

3.2.1 Long Kernel (AIM), SingleSIP-init . . . . . . . . . . . . . . . . 26

3.2.1.1 Variation of $d t$ and $\kappa \ldots \ldots \ldots \ldots . \ldots \ldots$

3.2.1.2 Variation of $\eta \ldots \ldots . \ldots . \ldots . \ldots 27$

3.2.1.3 Variation of $r_{\text {critmin }} \ldots \ldots \ldots \ldots . \ldots . \ldots 28$

3.2.1.4 Variation of $t_{\text {init }} \ldots \ldots \ldots \ldots$. . . . . . . . . . 29

3.2 .2 Long Kernel (AIM), $\nu_{\text {const }}$-init . . . . . . . . . . . . . . . . . . . . 31

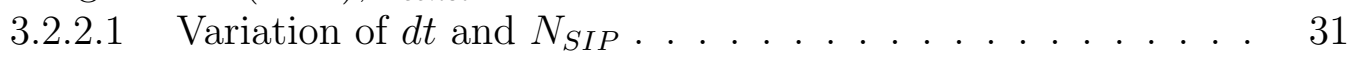

3.2.3 Long Kernel (AIM), $\nu_{d r a w}$-init . . . . . . . . . . . . . . . 32 
3.2.3.1 Variation of $d t$ and $N_{S I P} \ldots \ldots \ldots \ldots . \ldots . \ldots . \ldots 32$

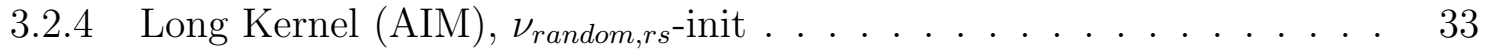

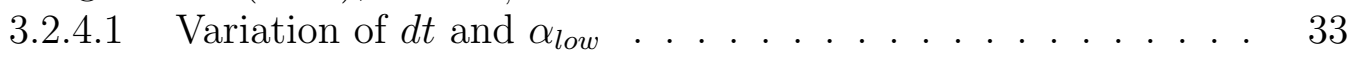

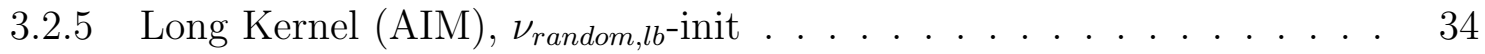

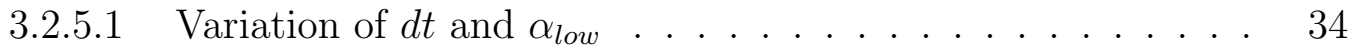

3.3 Hall Kernel (AIM) . . . . . . . . . . . . . . . . . . . . . . . . . 35

3.3 .1 Hall Kernel (AIM), SingleSIP-init . . . . . . . . . . . . . . . 36

3.3.1.1 Variation of $d t$ and $\kappa \ldots \ldots . \ldots . \ldots 36$

4 Simulation Results of All-or-Nothing Algorithm (AON) 37

4.1 Golovin Kernel $(\mathrm{AON})$. . . . . . . . . . . . . . . . . . . . . . . . . . . . . . 38

4.1.1 Golovin Kernel (AON), SingleSIP-init . . . . . . . . . . . . . . 39

4.1.1.1 Variation of $d t$ and $\kappa \ldots \ldots . \ldots . \ldots . \ldots . \ldots 39$

4.1.2 Golovin Kernel (AON), SingleSIP-init with $r_{\text {critmin }}=1.6 \mu \mathrm{m} \ldots . . \quad . \quad 40$

4.1.2.1 Variation of $d t$ and $\kappa \ldots \ldots . \ldots . \ldots 40$

4.1.3 Golovin Kernel (AON), Deterministic SingleSIP-init . . . . . . . . . . 41

4.1.3.1 Variation of $d t$ and $\kappa \ldots \ldots . \ldots . \ldots 41$

4.1.4 Golovin Kernel (AON), $\nu_{\text {const }}$-init . . . . . . . . . . . . . . . 42

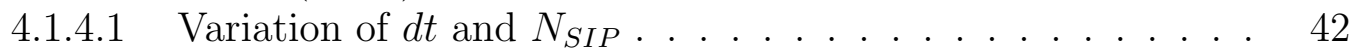

4.1.5 Golovin Kernel (AON), $\nu_{d r a w}$-init . . . . . . . . . . . . . . . . 43

4.1.5.1 Variation of $d t$ and $N_{S I P} \ldots \ldots \ldots \ldots . \ldots . \ldots . \ldots 43$

4.2 Long Kernel $(\mathrm{AON}) \ldots \ldots \ldots \ldots$. . . . . . . . . . . . . . . . . . . . . . . . . . . . . . .

4.2 .1 Long Kernel (AON), SingleSIP-init . . . . . . . . . . . . . . 45

4.2.1.1 Variation of $d t$ and $\kappa \ldots \ldots \ldots . \ldots . \ldots . \ldots 45$

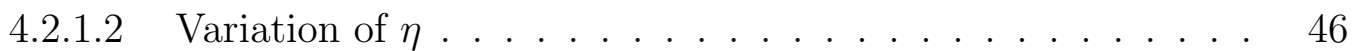

4.2.1.3 No multiple collections . . . . . . . . . . . . . . . . . . 47

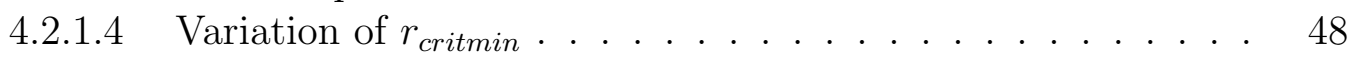

4.2.1.5 Variation of $t_{\text {init }} \ldots \ldots \ldots \ldots$. . . . . . . . . . 50

4.2.1.6 Order of combination processing . . . . . . . . . . 52

4.2.1.7 Hybrid init . . . . . . . . . . . . . . . . . 53

4.2 .2 Long Kernel $(\mathrm{AON})$, MultiSIP-init . . . . . . . . . . . . . 54

4.2 .3 Long Kernel $(\mathrm{AON}), \nu_{\text {const }}$-init . . . . . . . . . . . . . . . . 55

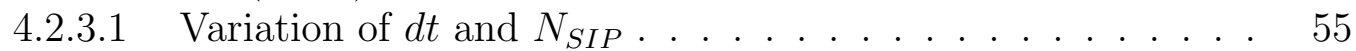

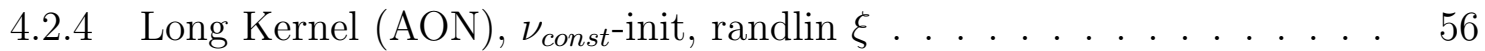

4.2.4.1 Variation of $d t$ and $N_{S I P} \ldots \ldots \ldots \ldots$. . . . . . 56

4.2.5 Long Kernel $(\mathrm{AON}), \nu_{\text {const }}$-init, $\operatorname{randlog} \xi \ldots \ldots \ldots \ldots$. . . . . . . . . . . . . . 57

4.2.5.1 Variation of $d t$ and $N_{S I P} \ldots \ldots \ldots \ldots \ldots$

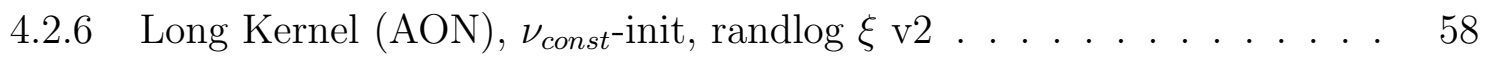

4.2.6.1 Variation of $d t$ and $N_{S I P} \ldots \ldots \ldots \ldots \ldots$

4.2 .7 Long Kernel $(\mathrm{AON}), \nu_{\text {draw }}$-init . . . . . . . . . . . . . . . . . 59

4.2.7.1 Variation of $d t$ and $N_{S I P} \ldots \ldots \ldots \ldots . \ldots . \ldots . \ldots 9$ 
4.2 .8 Long Kernel $(\mathrm{AON}), \nu_{\text {random } r s}$-init . . . . . . . . . . . . . . . 60

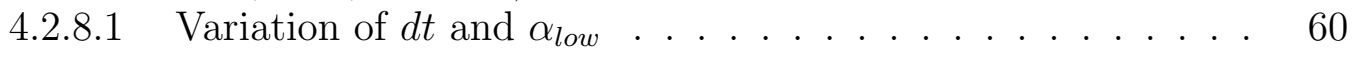

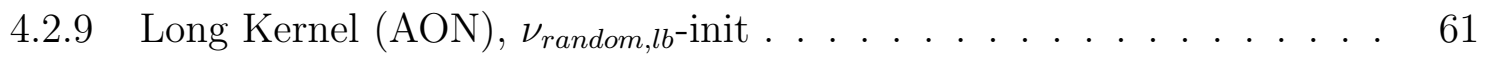

4.2.9.1 Variation of $d t$ and $\alpha_{\text {low }} \ldots \ldots \ldots \ldots$. . . . . . . . . 61

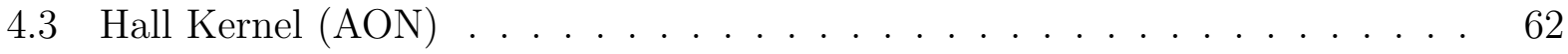

4.3.1 Hall Kernel $(\mathrm{AON})$, SingleSIP-init . . . . . . . . . . . . . . 63

4.3.1.1 Variation of $d t$ and $\kappa \ldots \ldots \ldots . \ldots \ldots$

4.3.1.2 Variation of $\eta \ldots \ldots . \ldots . \ldots . \ldots 64$

4.3.1.3 Variation of $r_{\text {critmin }} \ldots \ldots \ldots \ldots$. . . . . . . . . . . . . . . . . . . 65

4.3.2 Hall Kernel $(\mathrm{AON}), \nu_{\text {const }}$-init . . . . . . . . . . . . . . . 66

4.3.2.1 Variation of $d t$ and $N_{S I P} \ldots \ldots \ldots \ldots 6$

4.3 .3 Hall Kernel $(\mathrm{AON}), \nu_{\text {draw }}$-init . . . . . . . . . . . . . . . . . . 67

4.3.3.1 Variation of $d t$ and $N_{S I P} \ldots \ldots \ldots \ldots$. . . . . . . 67

4.4 Constant efficiency Kernel $(\mathrm{AON}) \ldots \ldots \ldots$

4.4.1 Collection efficiency $E_{c}=0.2$, SingleSIP-init . . . . . . . . . . . . 69

4.4.1.1 Variation of $d t$ and $\kappa \ldots \ldots \ldots$. . . . . . . . 69

4.4.2 Collection efficiency $E_{c}=1.0$, SingleSIP-init . . . . . . . . . . 70

4.4.2.1 Variation of $\kappa \ldots \ldots . \ldots 70$ 


\section{Introduction}

This document contains a systematic collection of figures. Any figure is a $4 \mathrm{x} 1$ panel showing the SIP number, droplet number and the second and third moment of the droplet mass distribution. All quantities are averages over 50 instances. Blocks 1, 2 and 3 show simulation results of the Remapping Algorithm (RMA), the Average Impact Algorithm (AIM) and the All-Or-Nothing algorithm (AON), respectively. Each block is divided into sections dealing with a specific kernel (Hall, Long and Golovin). The complete structure of the document becomes clear from looking at the table of contents which lists all presented sensitivity studies.

All plots showing the sensitivity to $d t$ use the colour and linestyle coding as given in the first row of the Table 1.

All plots showing the sensitivity to $\kappa$ use the colour coding as given in the second row of the Table 1.

All plots showing the sensitivity to $N_{S I P}$ use the colour coding as given in the second row of the Table 1.

A specific plot does not necessarily show curves for all parameter values listed in Table 1.

Moreover, the y-scale runs in all $N_{S I P}$-plots (top row) from 0 to 600 (and sometimes t0 400 ) and the plots miss to show simulations with larger $N_{S I P}$. It occurs quite often that several curves overlap and it is not clear which curves are actually not shown or which are just hidden by other curves. In the case, where $\kappa$ or $N_{S I P}$ is varied, the $N_{S I P}$-plot helps to see which curves are plotted. For other sensitivities, it is most often not evident which parameter values are actually shown.

Table 1: Colour coding for the various sensitivity simulations series. By default, the linestyle is solid. For the values listed with a ${ }^{*}$-symbol, dotted curves are used.

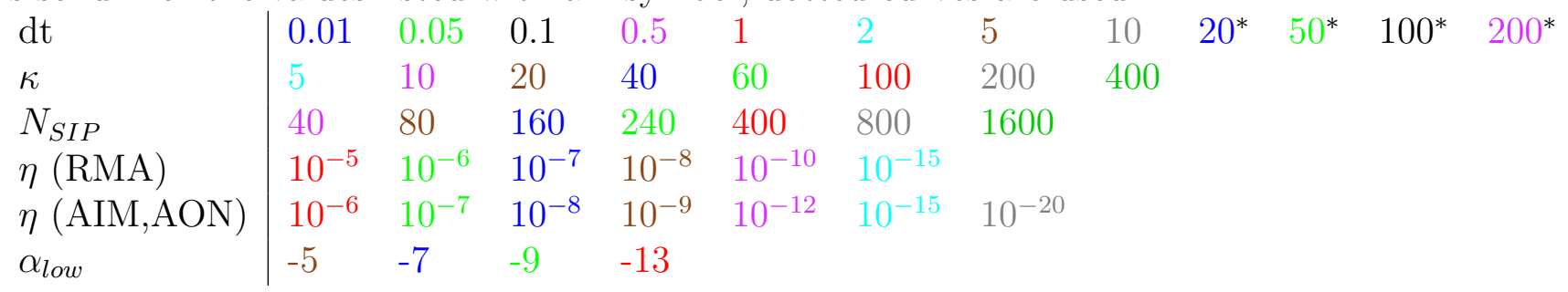




\section{Simulation Results of Remapping Algorithm (RMA)}

Block 2 displays RMA simulation results for the Golovin and Long kernel (no Hall simulations are included in this document).

The default settings are $\eta=10^{-8}$ (weak threshold) and $r_{\text {critmin }}=1.6 \mu \mathrm{m}$.

For Golovin RMA simulations the default is: $d t=1 \mathrm{~s}, \kappa=40$ and the algorithm version without Reduction Limiter.

For Long RMA simulations the default is: $d t=0.1 \mathrm{~s}, \kappa=10$ and the algorithm version with Reduction Limiter.

For the algorithm with Reduction Limiter the parameter $\tilde{\gamma}=0.1$. 


\subsection{Golovin Kernel (RMA)}

The following sections show Golovin RMA results for the regular algorithm, the version with Reduction Limiter, the version with Update on the Fly $\left(\mathrm{OTF}_{s}\right.$, starting with the smallest SIPs) and the version with Update on the Fly $\left(\mathrm{OTF}_{l}\right.$, starting with the largest SIPs) 


\subsubsection{Golovin Kernel (RMA), regular RMA version}

2.1.1.1 Variation of $d t$ and $\kappa$
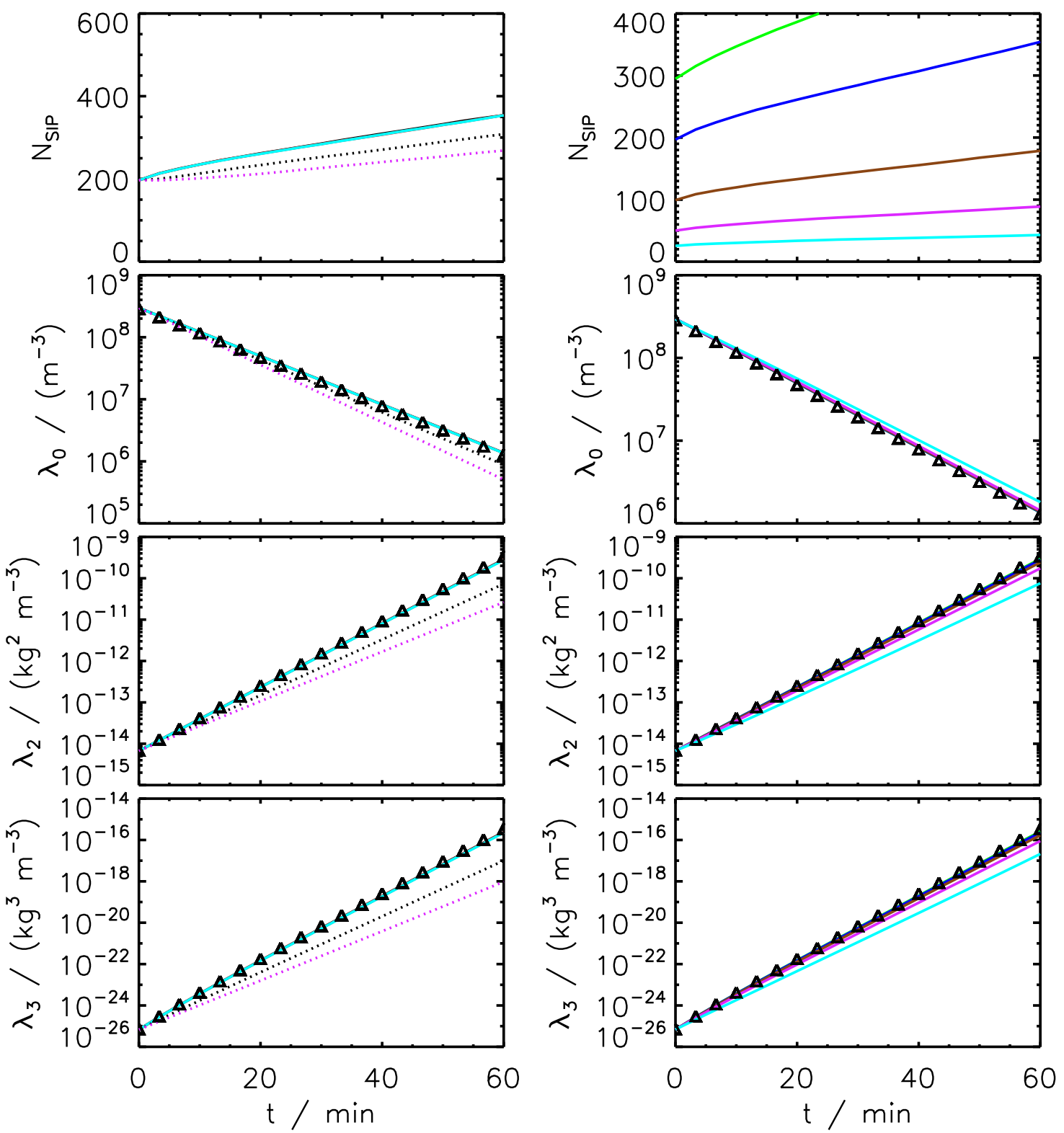

Figure 1: GOL RMA: Variation of time step $d t$ (left) and bin resolution $\kappa$ (right). 


\subsubsection{Variation of $\eta$}

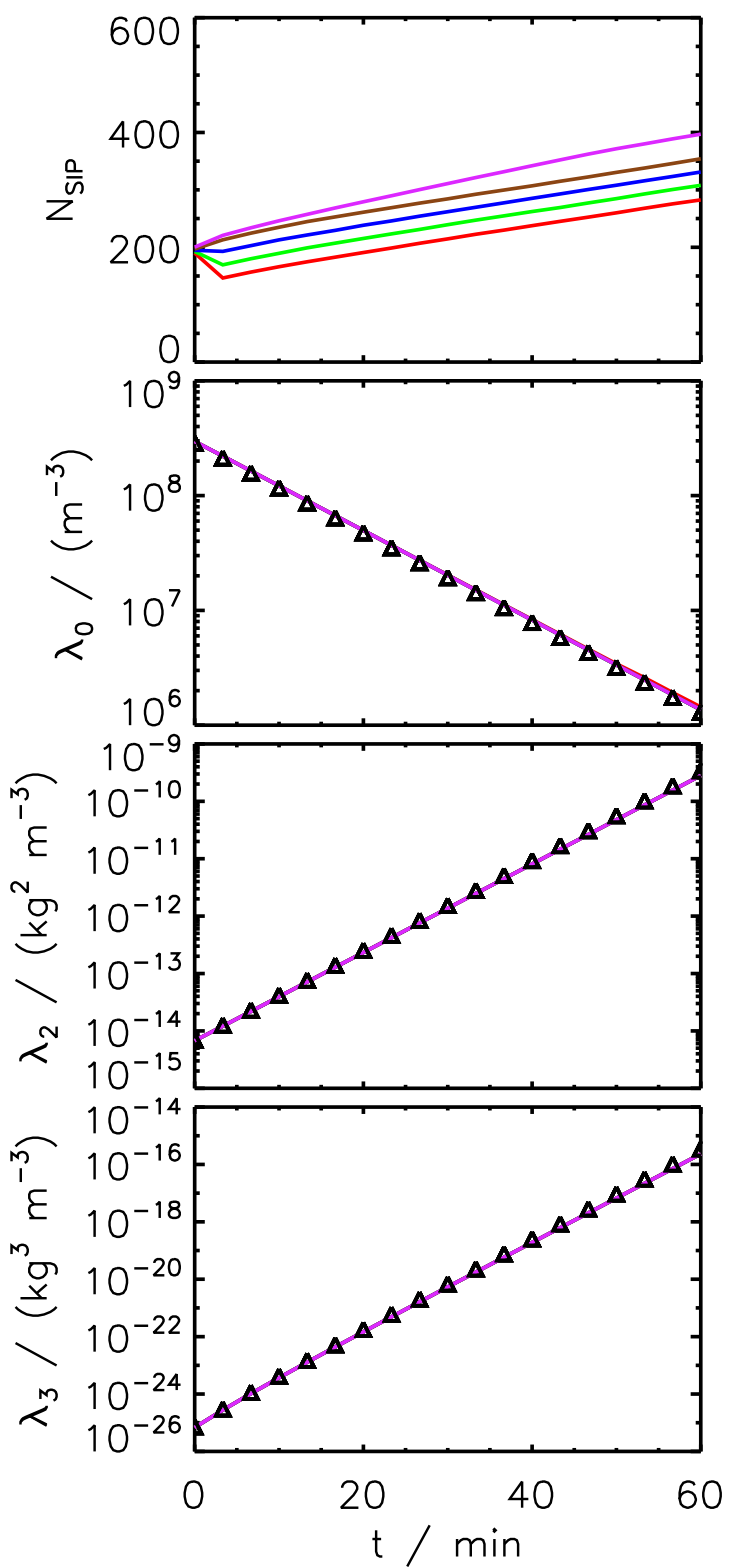

Figure 2: GOL RMA: Variation of (weak) threshold $\eta$. 
2.1.1.3 Strict threshold, variation of $\eta \quad$ No SIPs are generated from bins with $M_{b i n, l}<$ $M_{\text {critmin }}=\eta \lambda_{1}$. This is the only plot in the document, where $\lambda_{1}$ is shown. For too large $\eta$, mass is not conserved.
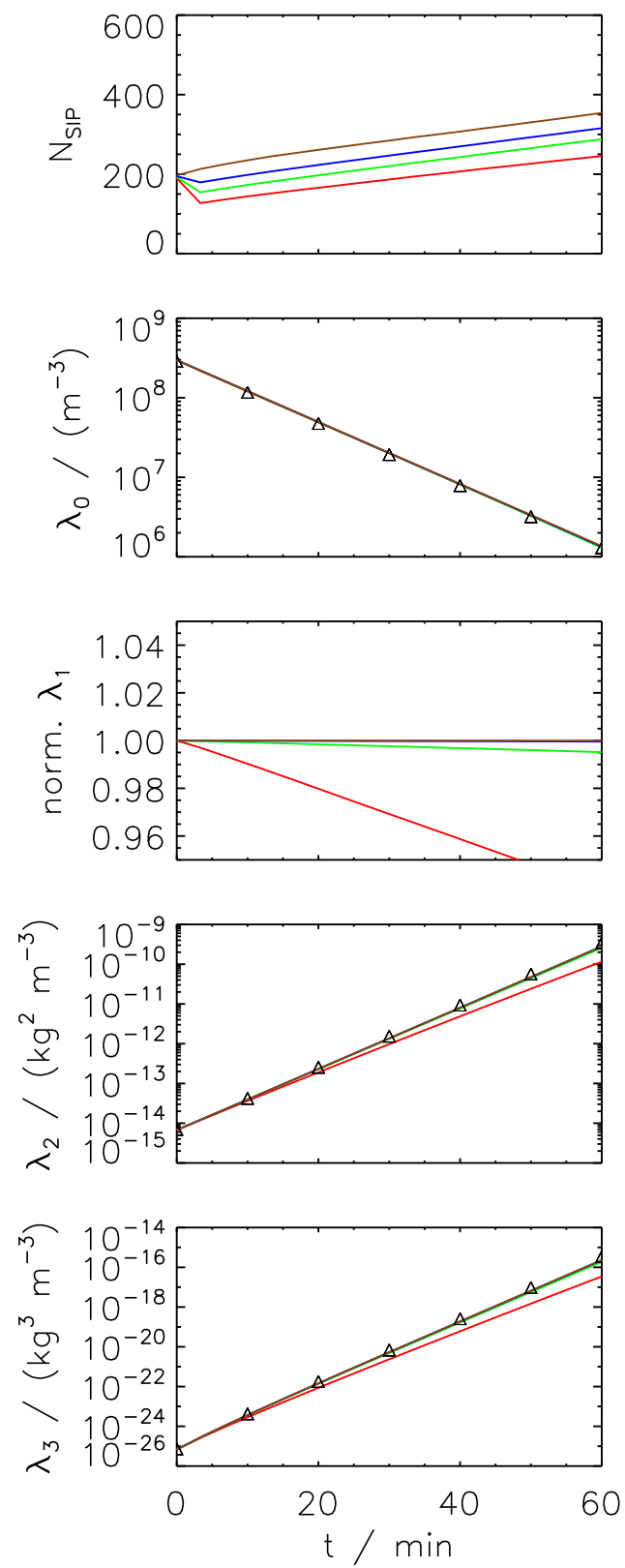

Figure 3: GOL RMA: Variation of strict threshold $\eta$. 


\subsubsection{Golovin Kernel (RMA), RMA with Reduction Limiter}

\subsubsection{Variation of $d t$ and $\kappa$}
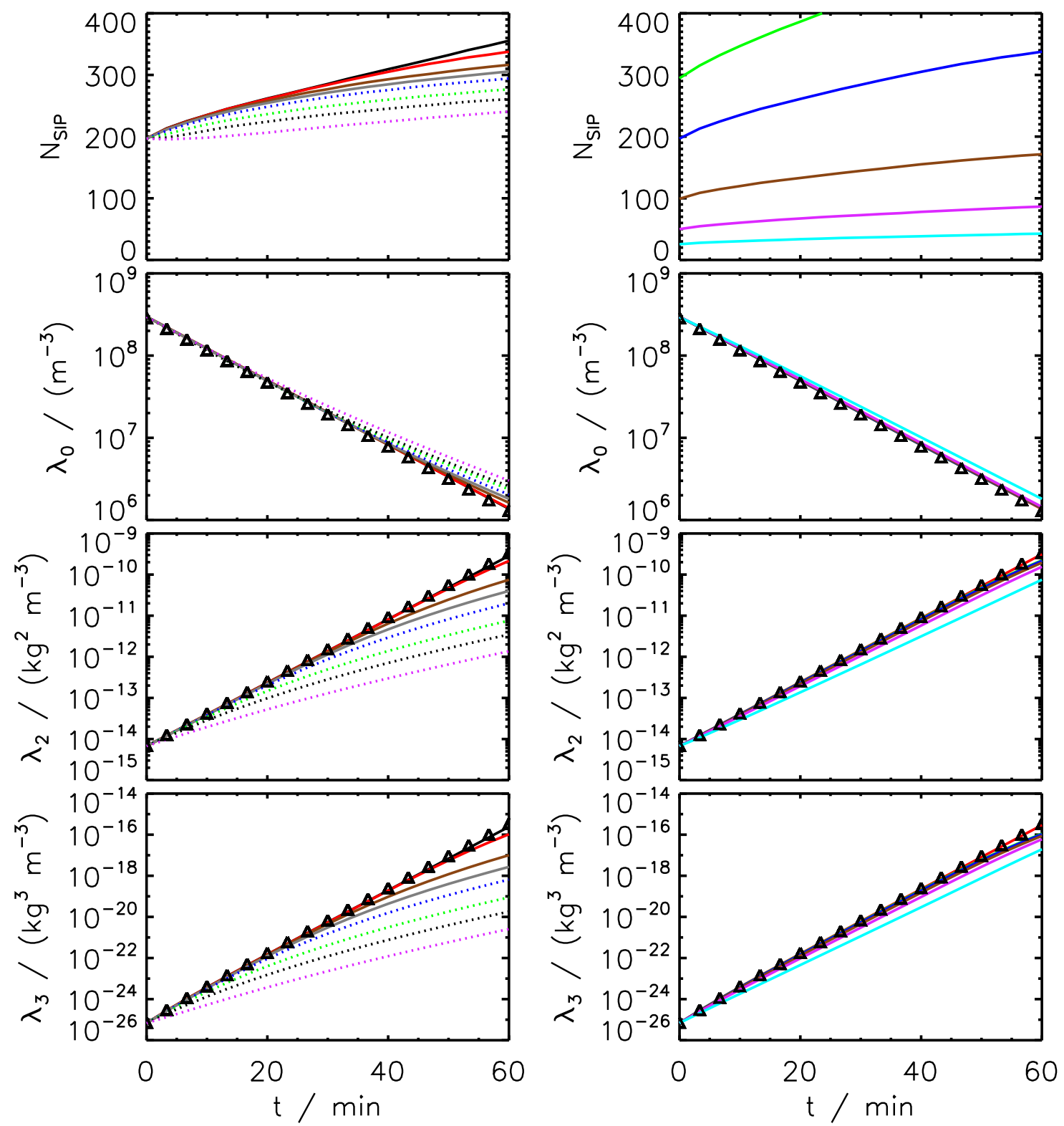

Figure 4: GOL RMA (with Reduction Limiter): Variation of time step $d t$ (left) and bin resolution $\kappa$ (right). 


\subsubsection{Variation of $r_{\text {critmin }}$ and $\eta$}
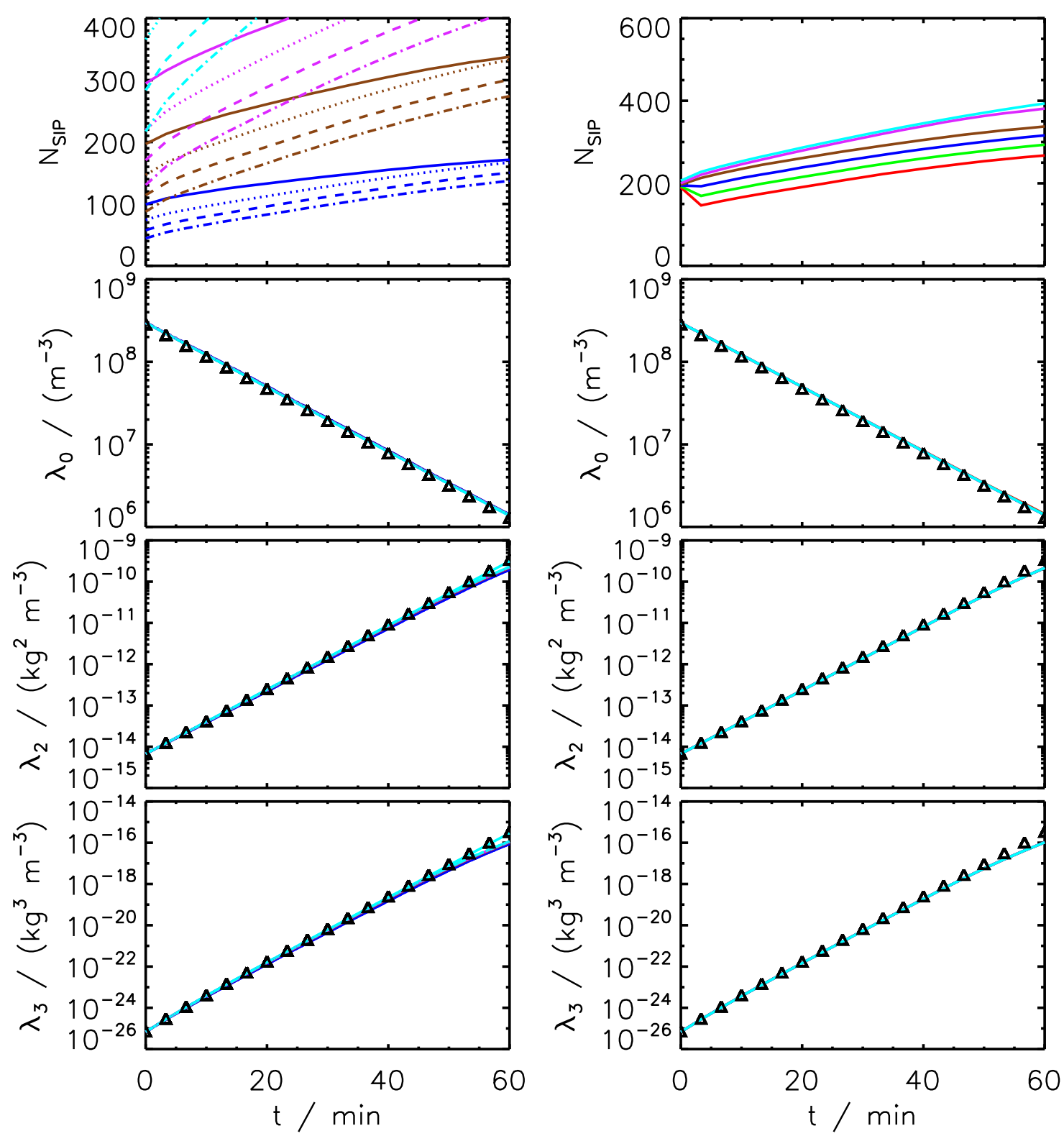

Figure 5: GOL RMA (with Reduction Limiter): Variation of $r_{\text {critmin }}$ (left). Colours indicate the $\kappa$-value and the linestyle the $r_{\text {critmin }}$-value: $0.6 \mu \mathrm{m}$ (solid), $1.6 \mu \mathrm{m}$ (dotted), $3.0 \mu \mathrm{m}$ (dashed), $5.0 \mu \mathrm{m}$ (dash-dotted). Variation of threshold $\eta$ (right). 


\subsubsection{Variation of $\tilde{\gamma}$}

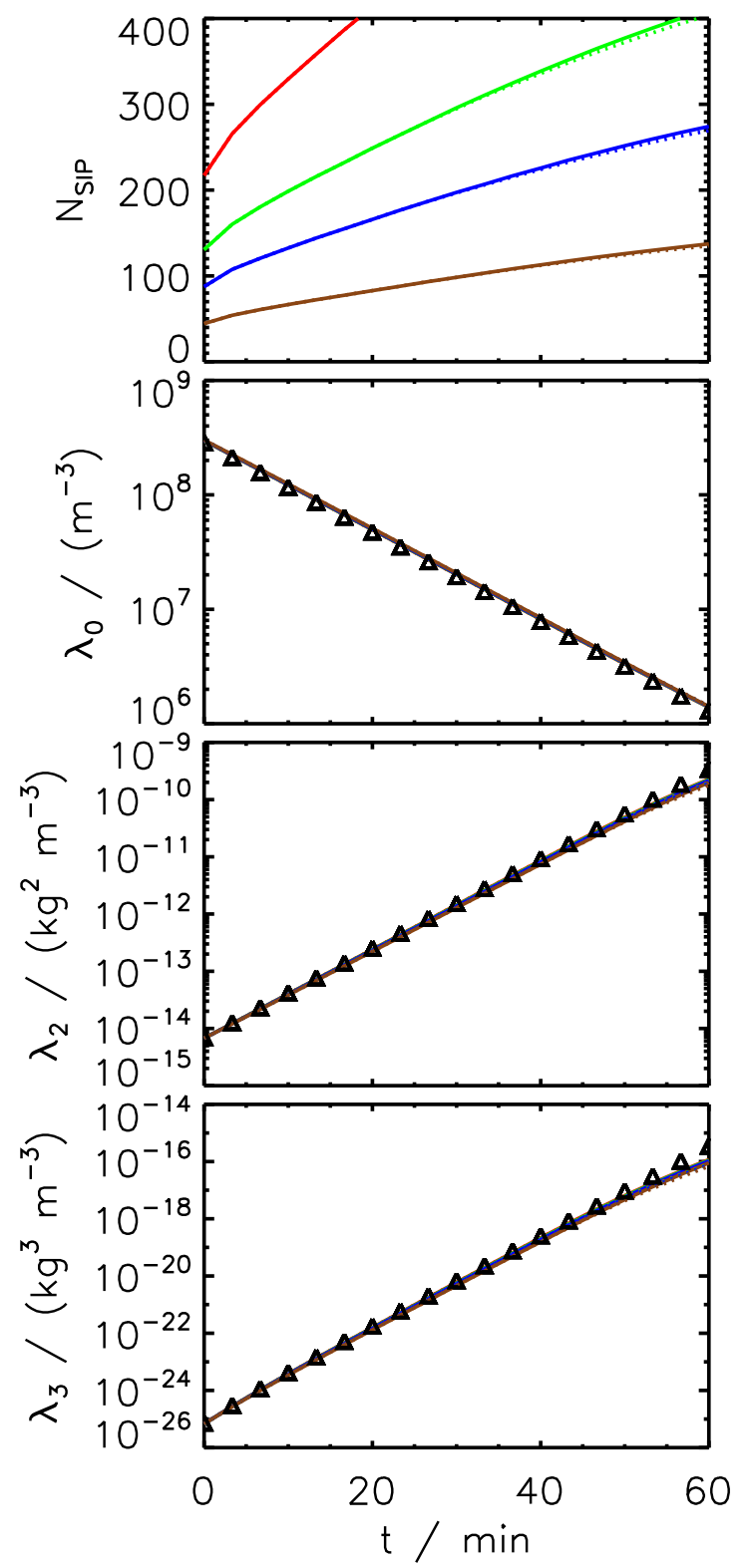

Figure 6: GOL RMA (with Reduction Limiter): two values of scaling factor $\tilde{\gamma}$ (default value 0.1 solid and value 0.5 dotted) for various $\kappa$. 


\subsubsection{Golovin Kernel (RMA), RMA with Update on the Fly $\mathrm{OTF}_{s}$}

\subsubsection{Variation of $d t$ and $\kappa$}
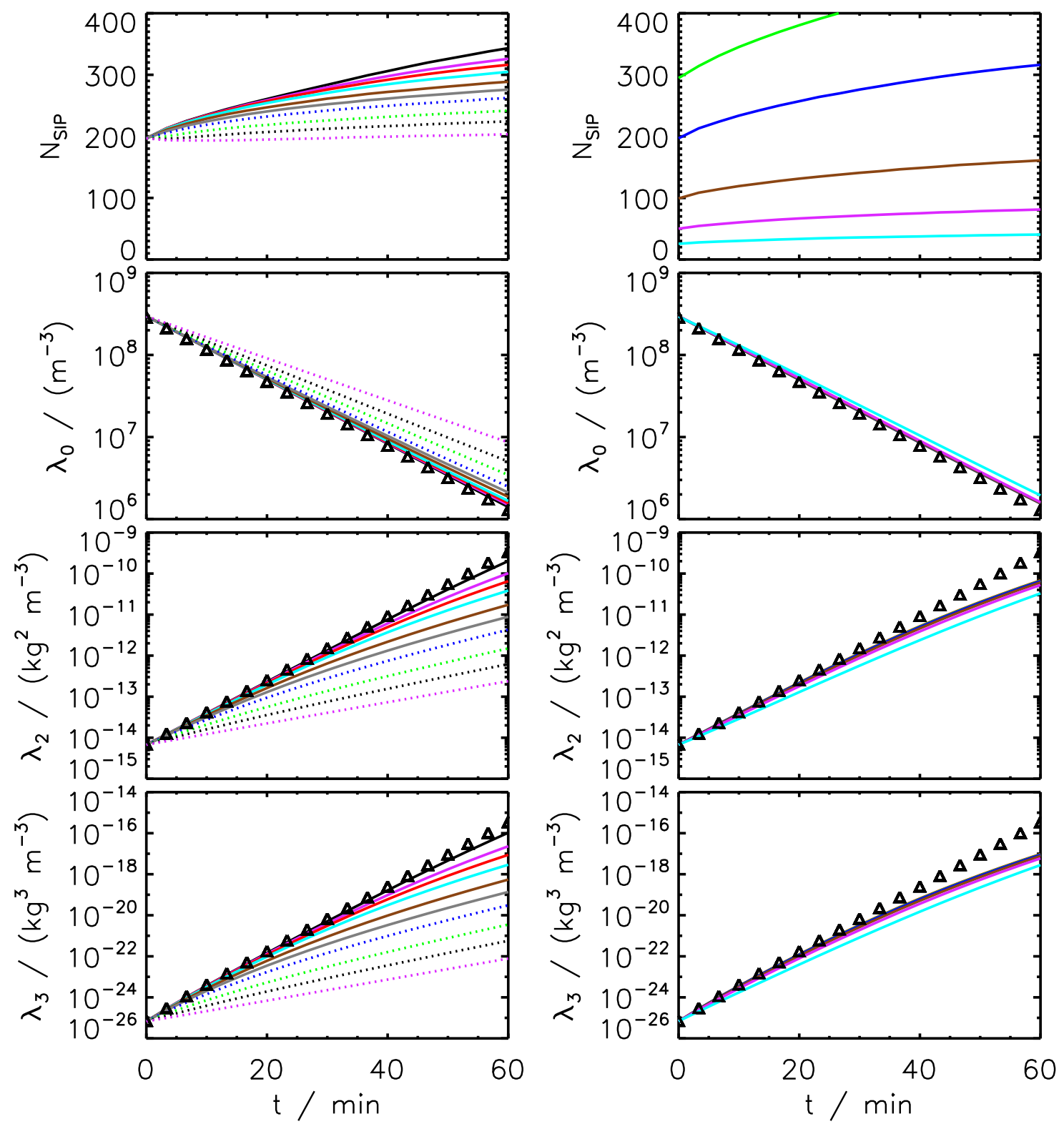

Figure 7: GOL RMA (with $\mathrm{OTF}_{s}$ ): Variation of time step $d t$ (left) and bin resolution $\kappa$ (right). 
2.1.4 Golovin Kernel (RMA), RMA with Update on the Fly $\mathrm{OTF}_{l}$

2.1.4.1 Variation of $d t$ and $\kappa$
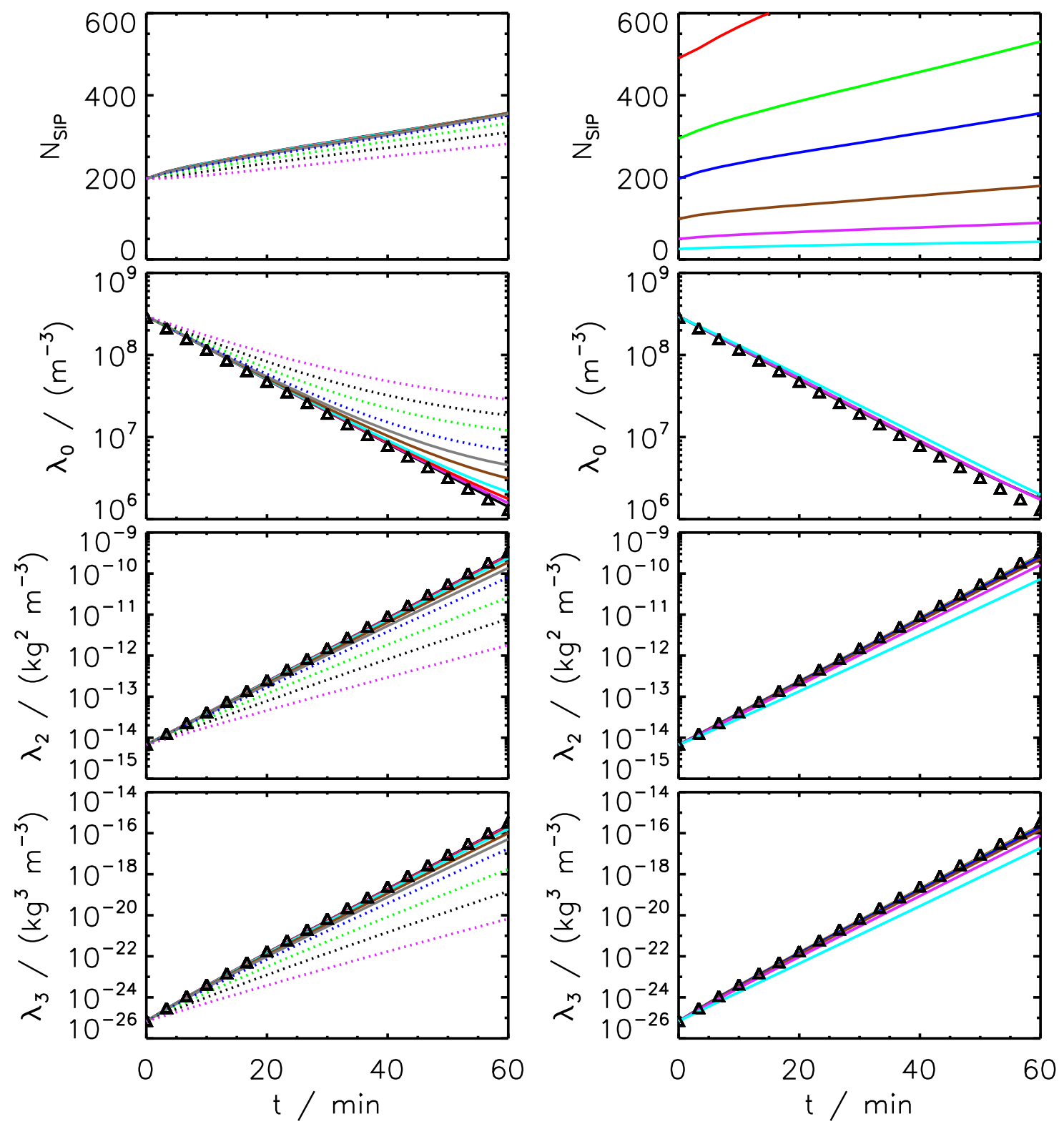

Figure 8: GOL RMA (with $\mathrm{OTF}_{l}$ ): Variation of time step $d t$ (left) and bin resolution $\kappa$ (right). 


\subsection{Long Kernel (RMA)}

The following sections show Long RMA results for the algorithm with Redcution Limiter and for the version with Update on the Fly $\left(\mathrm{OTF}_{l}\right.$, starting with the largest SIPs) 


\subsubsection{Long Kernel, RMA with Reduction Limiter}

\subsubsection{Variation of $d t$ and $\kappa$}
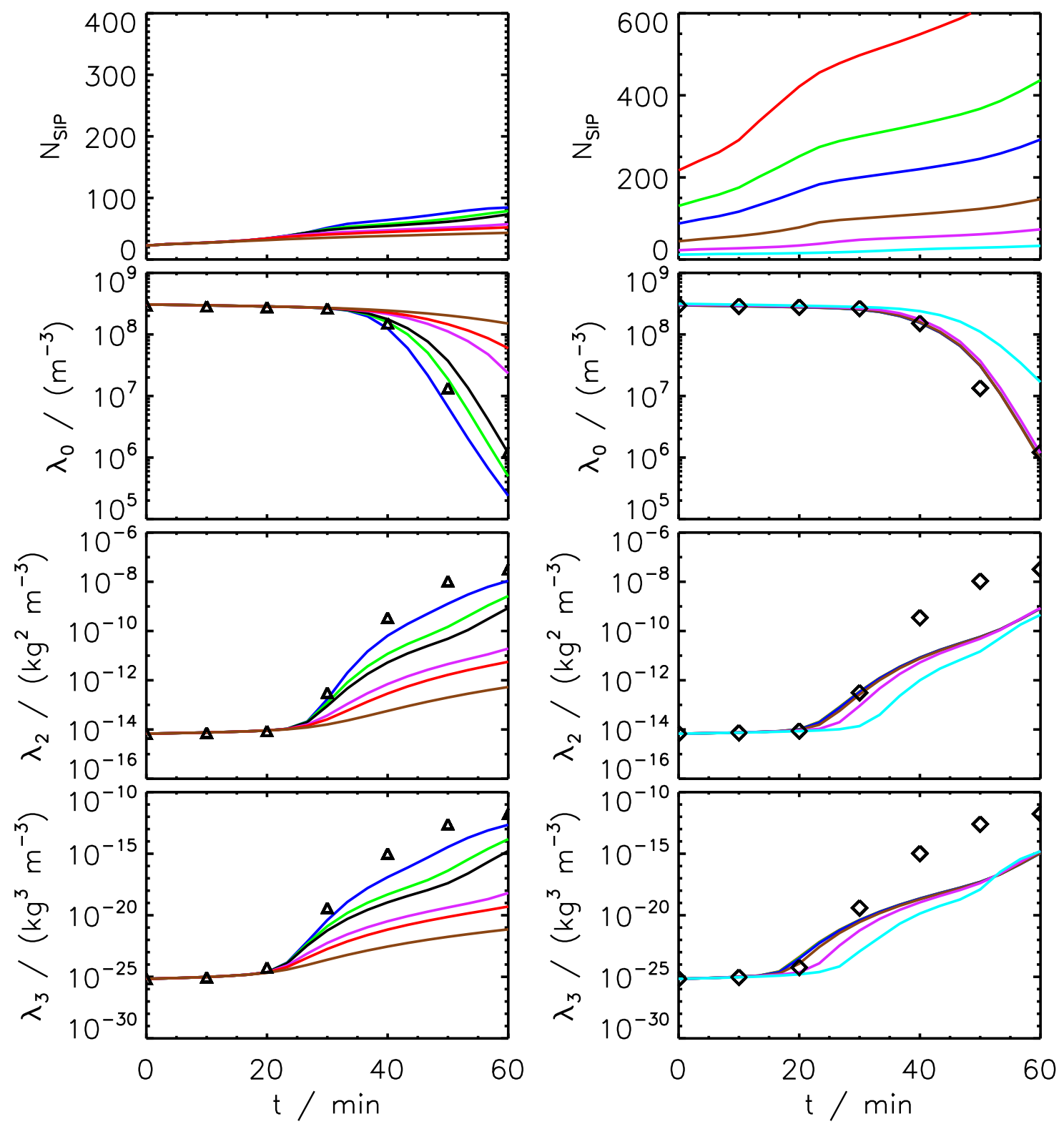

Figure 9: LONG RMA (with Reduction Limiter): Variation of time step $d t$ (left) and bin resolution $\kappa$ (right). 


\subsubsection{Variation of $t_{i n i t}$ and $\eta$}
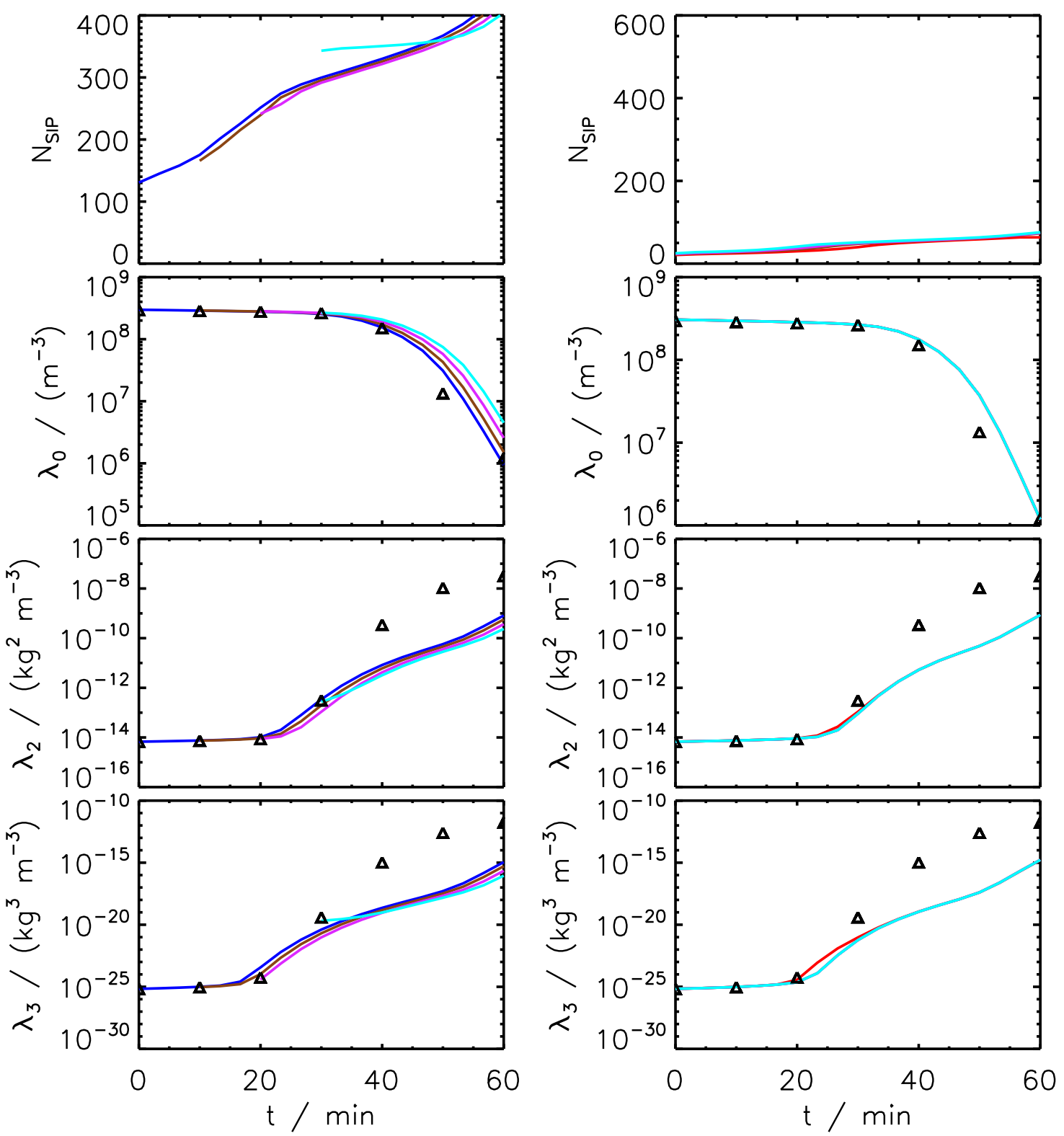

Figure 10: LONG RMA (with Reduction Limiter): Variation of $t_{\text {init }}$ at $\kappa=60$ (left). The colours are as follows: $t_{\text {init }} / \mathrm{min}=0$ (blue), 10 (brown), 20 (magenta) and 30 (cyan). Variation of threshold $\eta$ (right). 


\subsubsection{Long Kernel, RMA with Update on the Fly $\mathrm{OTF}_{l}$}

\subsubsection{Variation of $d t$ and $\kappa$}
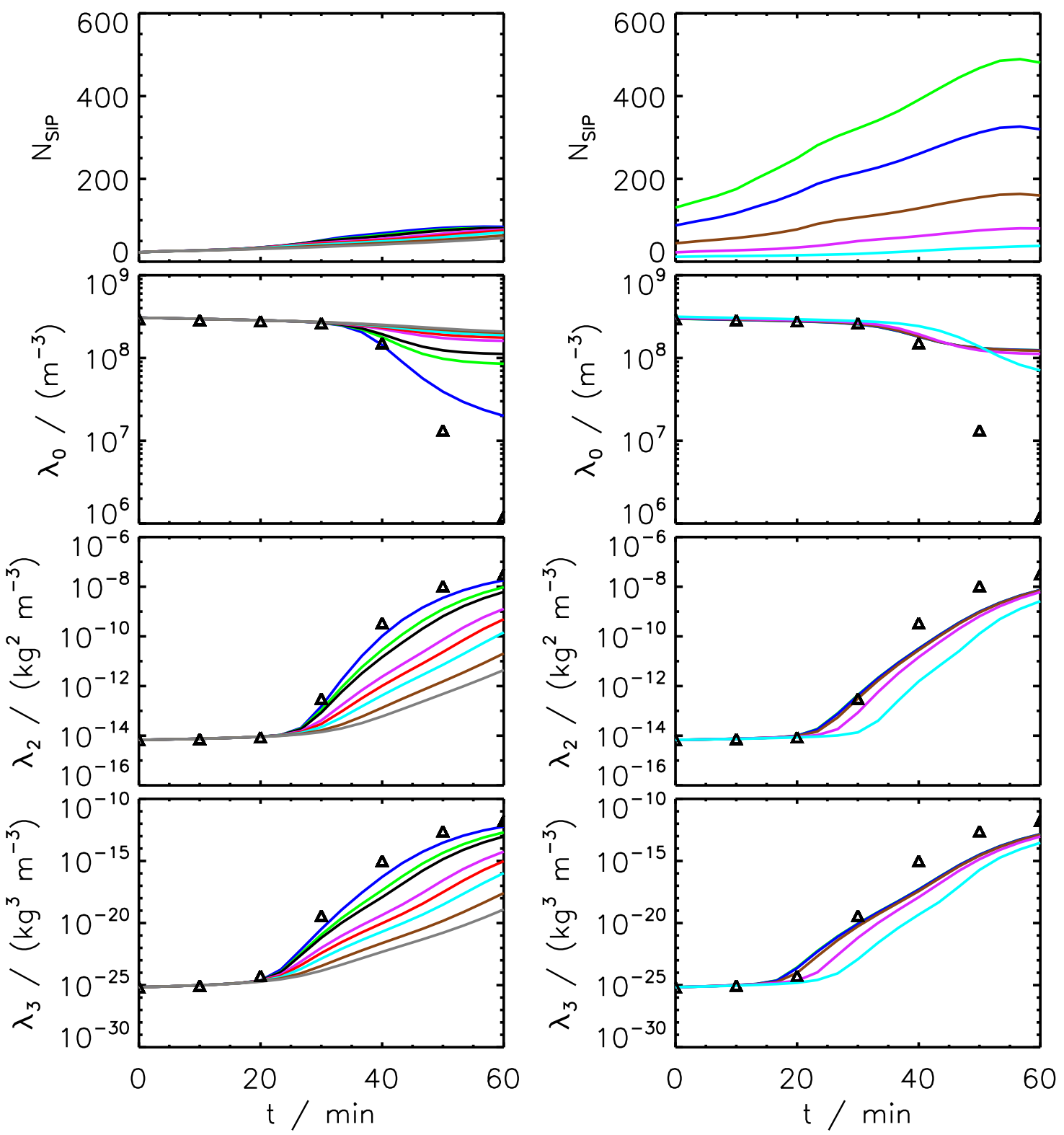

Figure 11: LONG RMA (with $\mathrm{OTF}_{l}$ ): Variation of time step $d t$ (left) and bin resolution $\kappa$ (right). 


\section{Simulation Results of Average Impact Algorithm (AIM)}

Block 3 displays AIM simulation results for the Golovin, Long and Hall kernel.

All AIM simulations use as default $d t=10 \mathrm{~s}$.

The SingleSIP initialisation method uses as default $\kappa=40, r_{\text {critmin }}=0.6 \mu \mathrm{m}$ and $\eta=10^{-8}$ and is probabilistic.

The $\nu_{\text {const }}$ and $\nu_{\text {draw }}$ initialisation methods use as default $N_{S I P}=160$. 


\subsection{Golovin Kernel (AIM)}

The following sections show Golovin AIM results for three types of init methods. 


\subsubsection{Golovin Kernel (AIM), SingleSIP-init}

\subsubsection{Variation of $d t$ and $\kappa$}
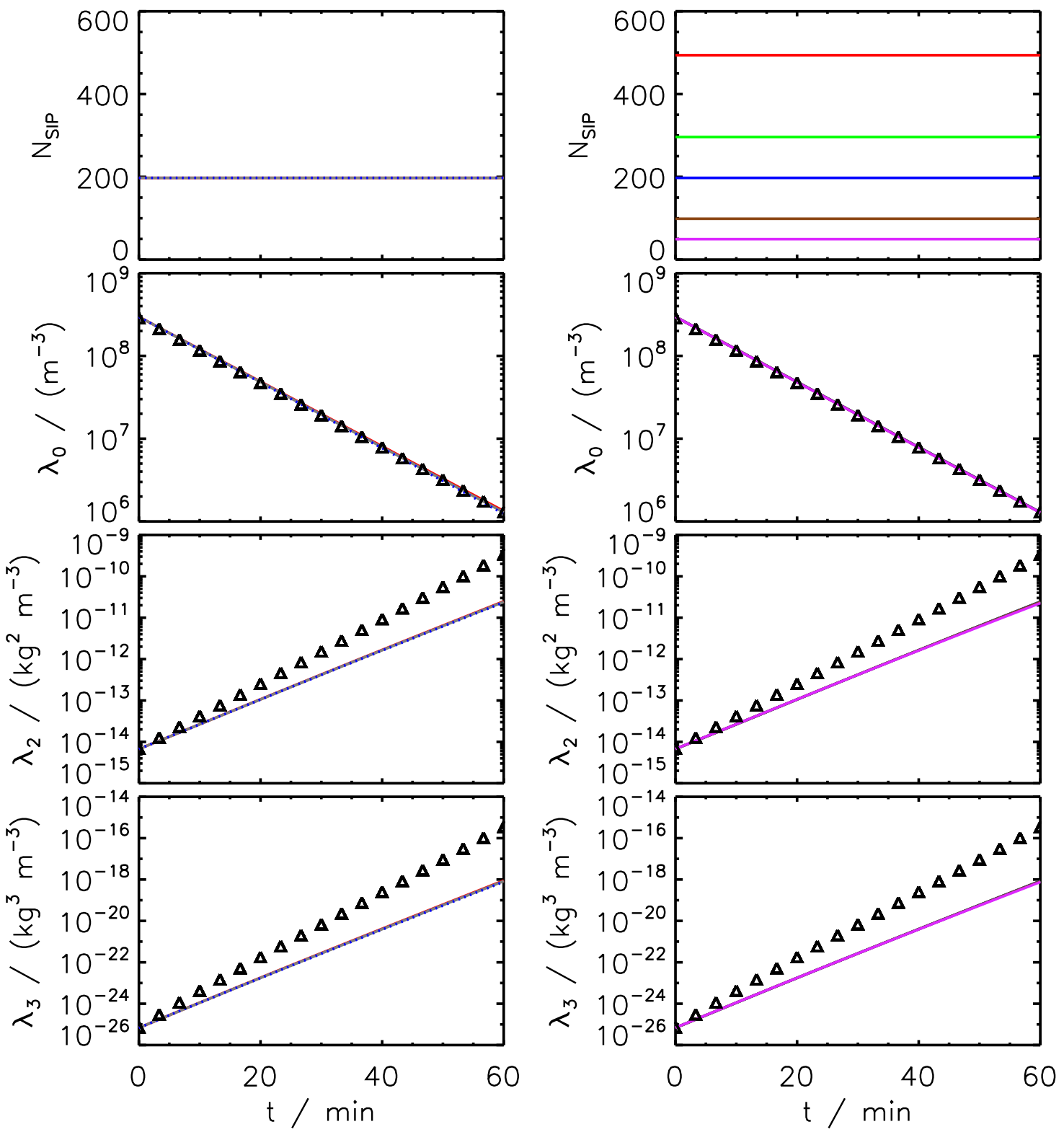

Figure 12: GOL AIM: Variation of time step $d t$ (left) and bin resolution $\kappa$ (right). 


\subsubsection{Golovin Kernel (AIM), $\nu_{\text {const }}$-init}

\subsubsection{Variation of $N_{S I P}$}

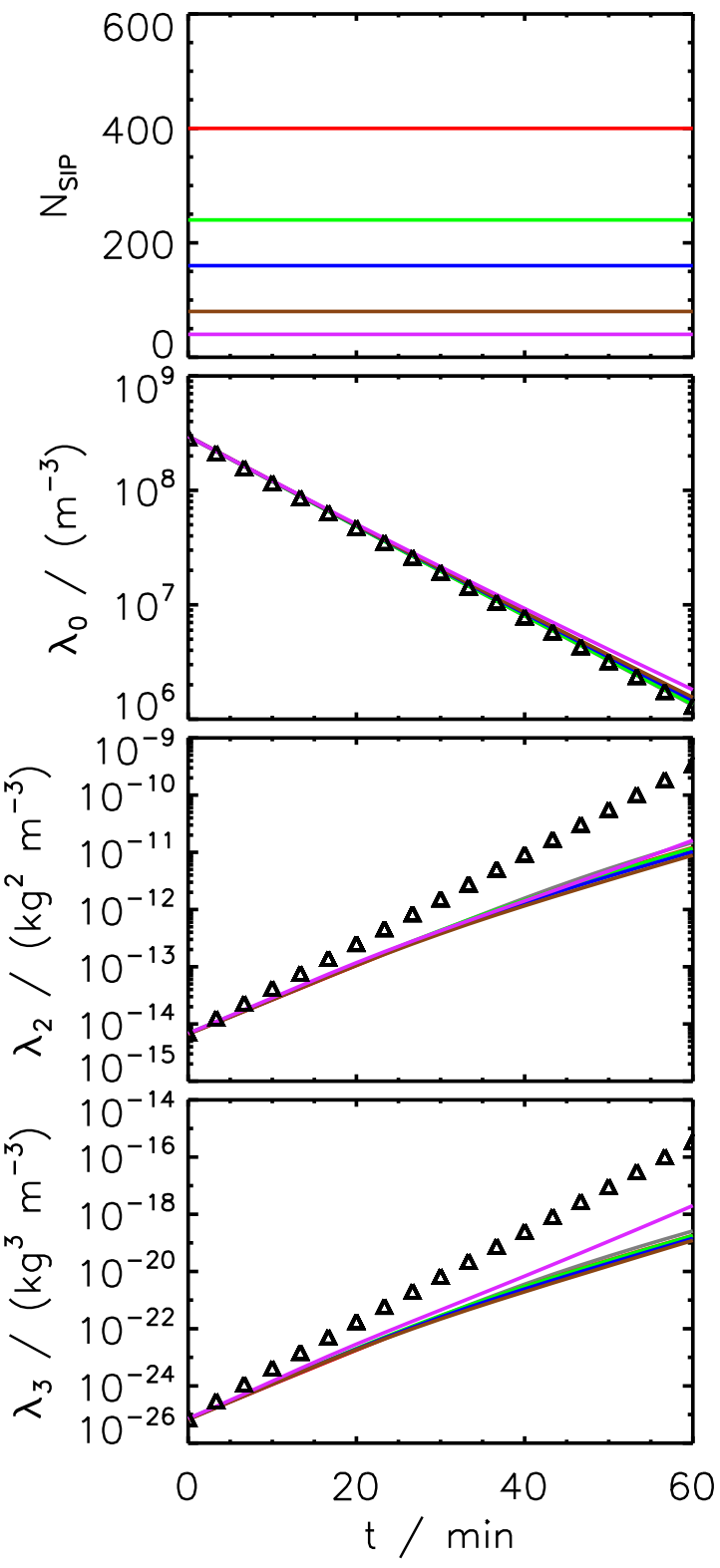

Figure 13: GOL AIM: Variation of SIP number $N_{S I P}$. 


\subsubsection{Golovin Kernel (AIM), $\nu_{d r a w}$-init}

\subsubsection{Variation of $N_{S I P}$}

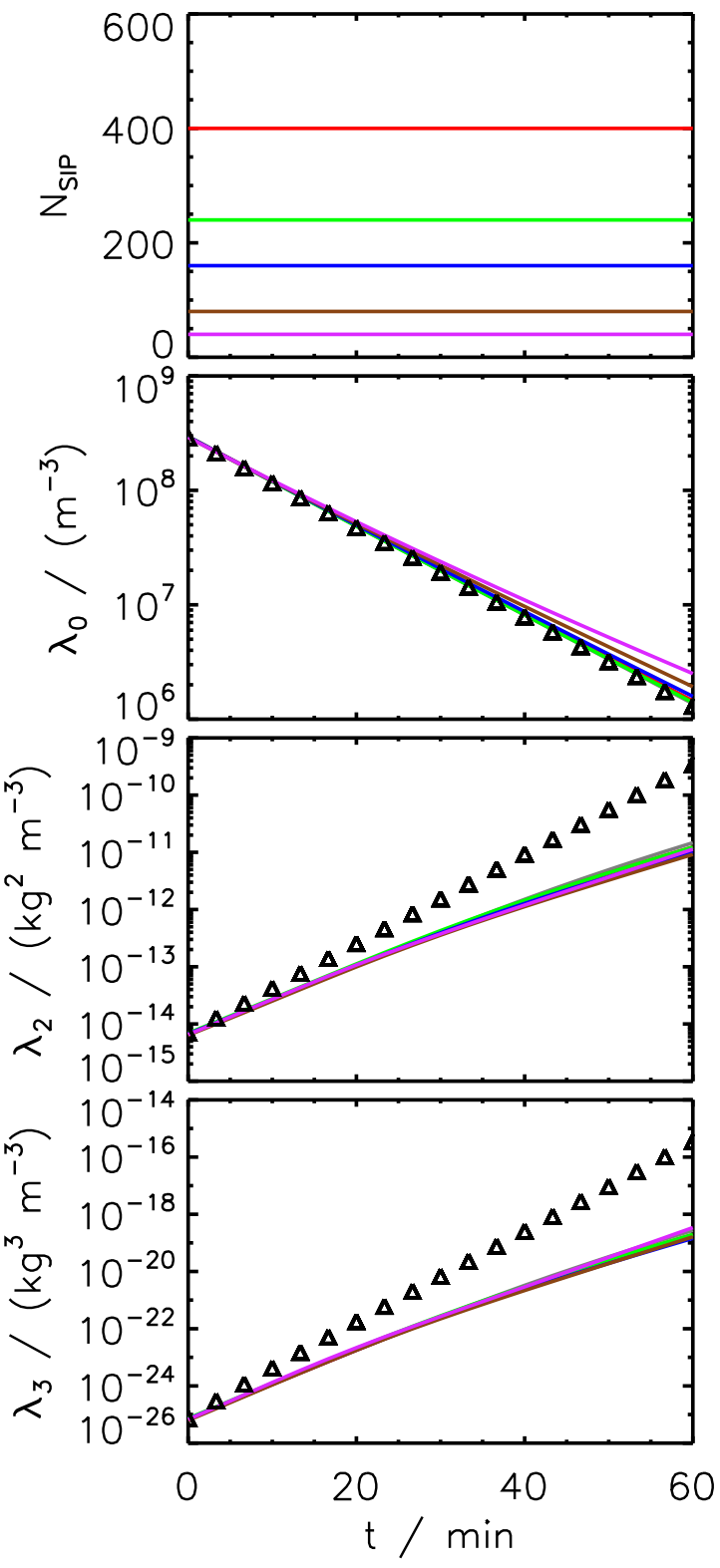

Figure 14: GOL AIM: Variation of SIP number $N_{S I P}$. 


\section{$3.2 \quad$ Long Kernel (AIM)}

The following sections show Long AIM results for five types of init methods. 


\subsubsection{Long Kernel (AIM), SingleSIP-init}

\subsubsection{Variation of $d t$ and $\kappa$}
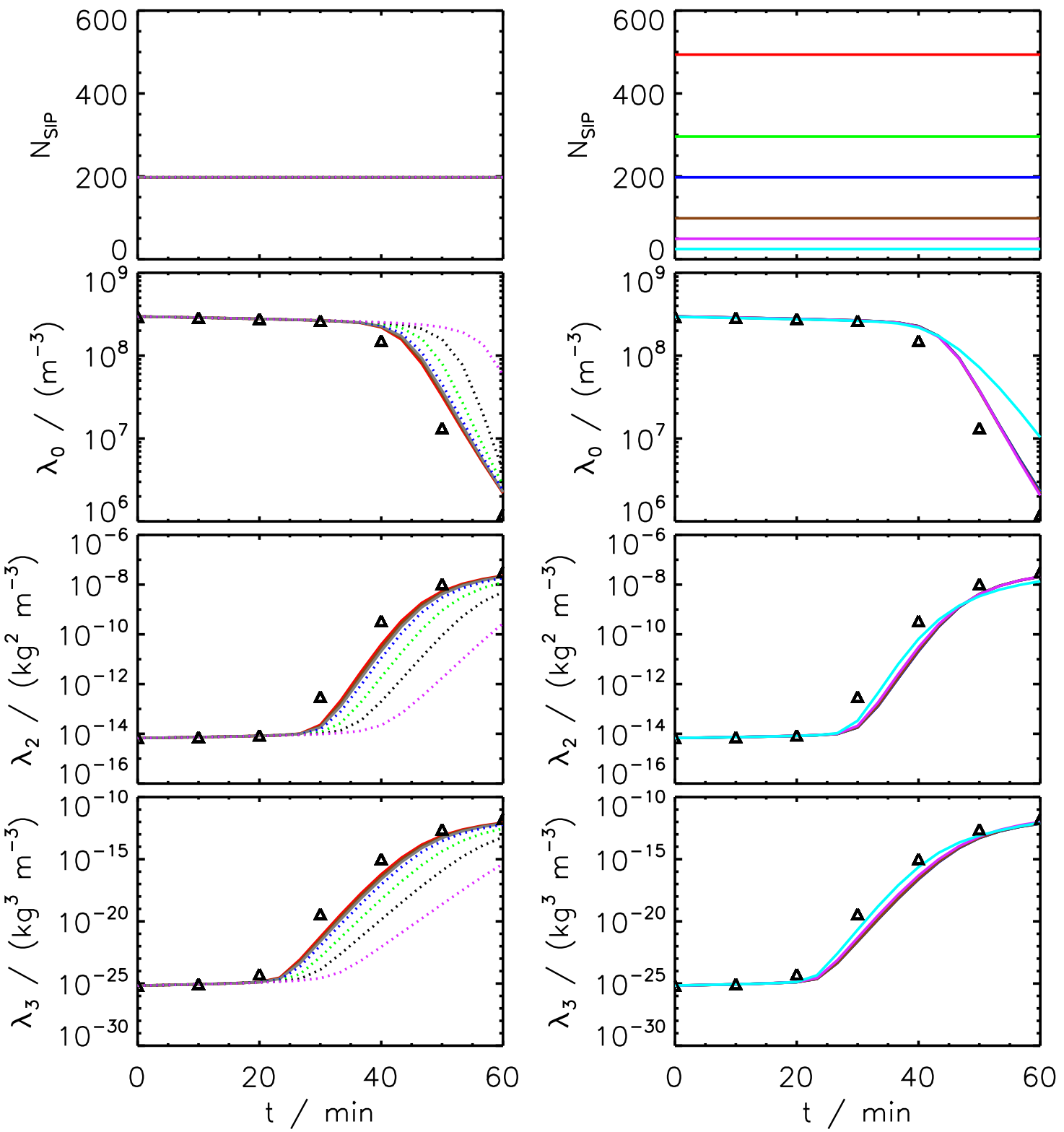

Figure 15: LONG AIM: Variation of time step $d t$ (left) and bin resolution $\kappa$ (right). 


\subsubsection{Variation of $\eta$}

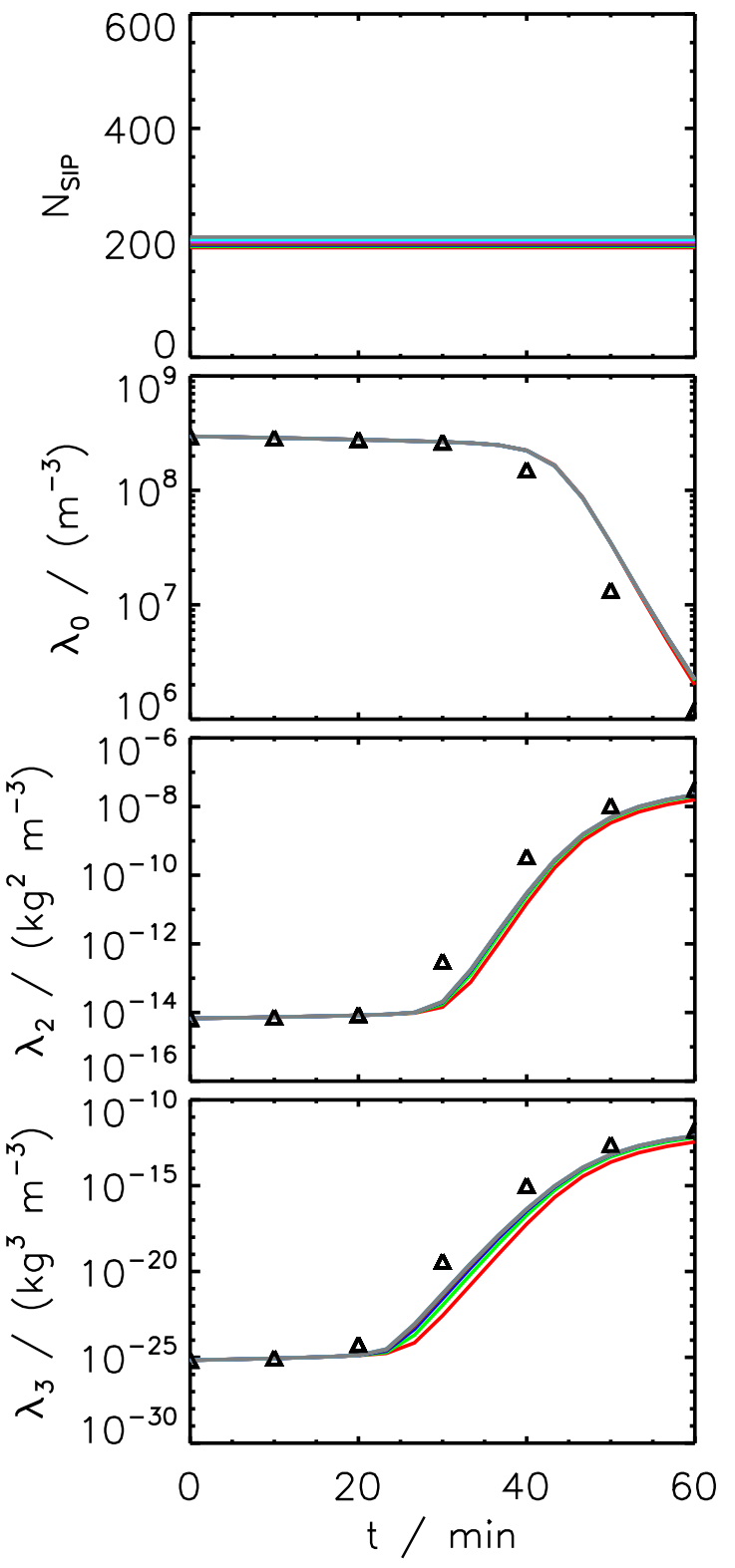

Figure 16: LONG AIM: Variation of threshold $\eta$. 


\subsubsection{Variation of $r_{\text {critmin }}$}
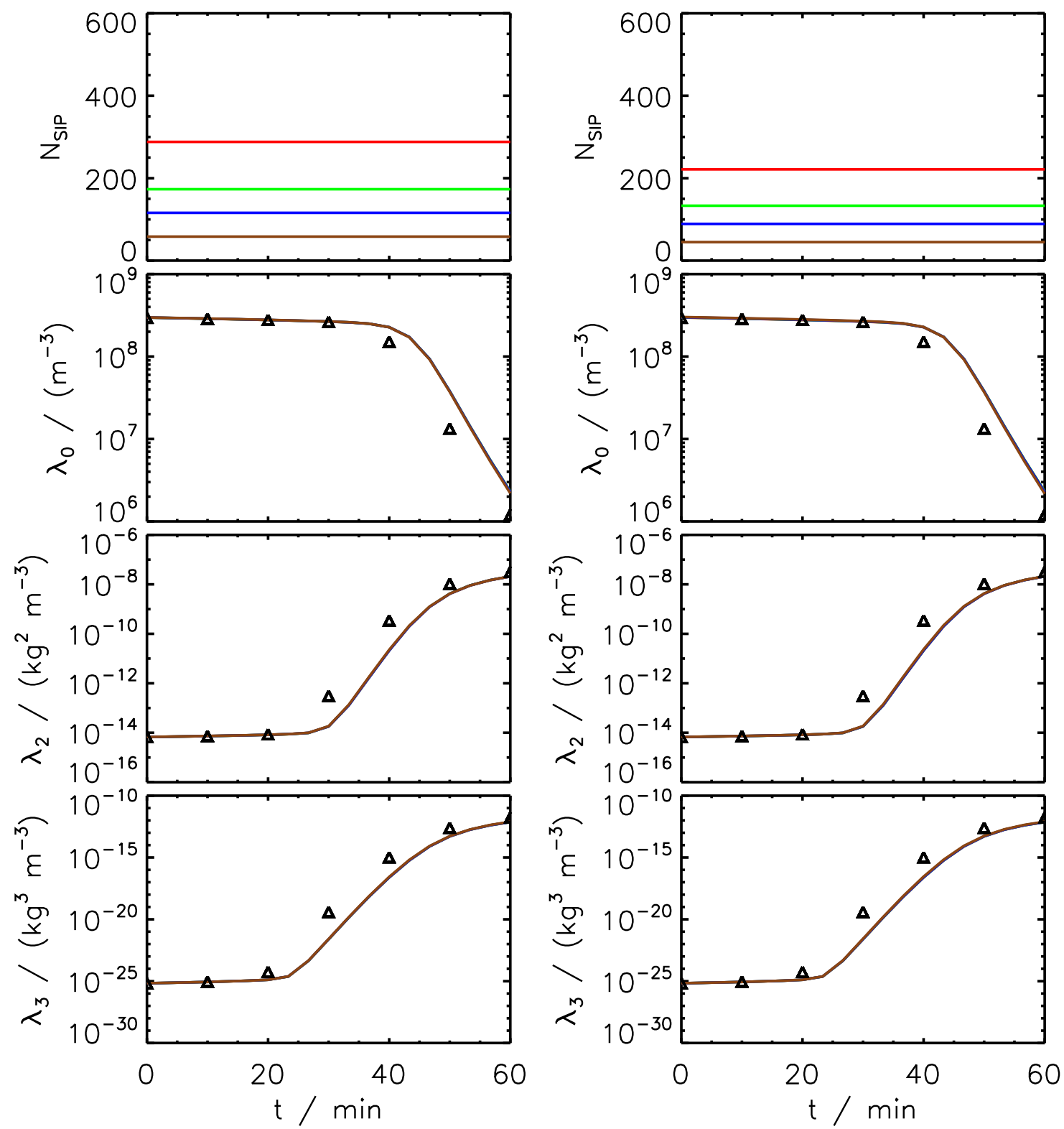

Figure 17: LONG AIM: Variation of bin resolution $\kappa$ at $r_{\text {critmin }}=3.0 \mu \mathrm{m}$ (left) and $5.0 \mu \mathrm{m}$ (right). 


\subsubsection{Variation of $t_{\text {init }}$}
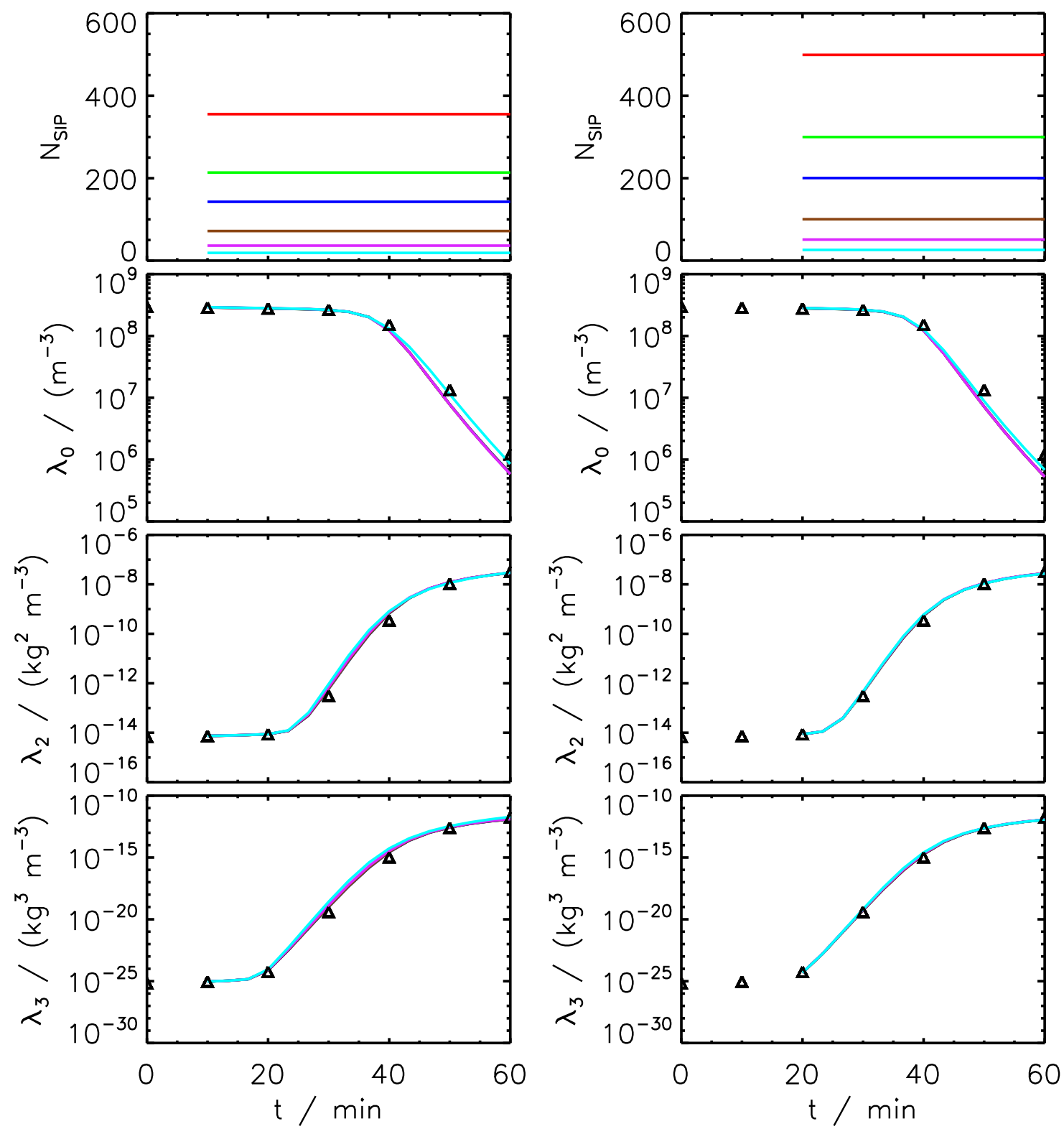

Figure 18: LONG AIM: Variation of bin resolution $\kappa$ at $t_{\text {init }}=10 \mathrm{~min}$ (left) and $20 \mathrm{~min}$ (right). 


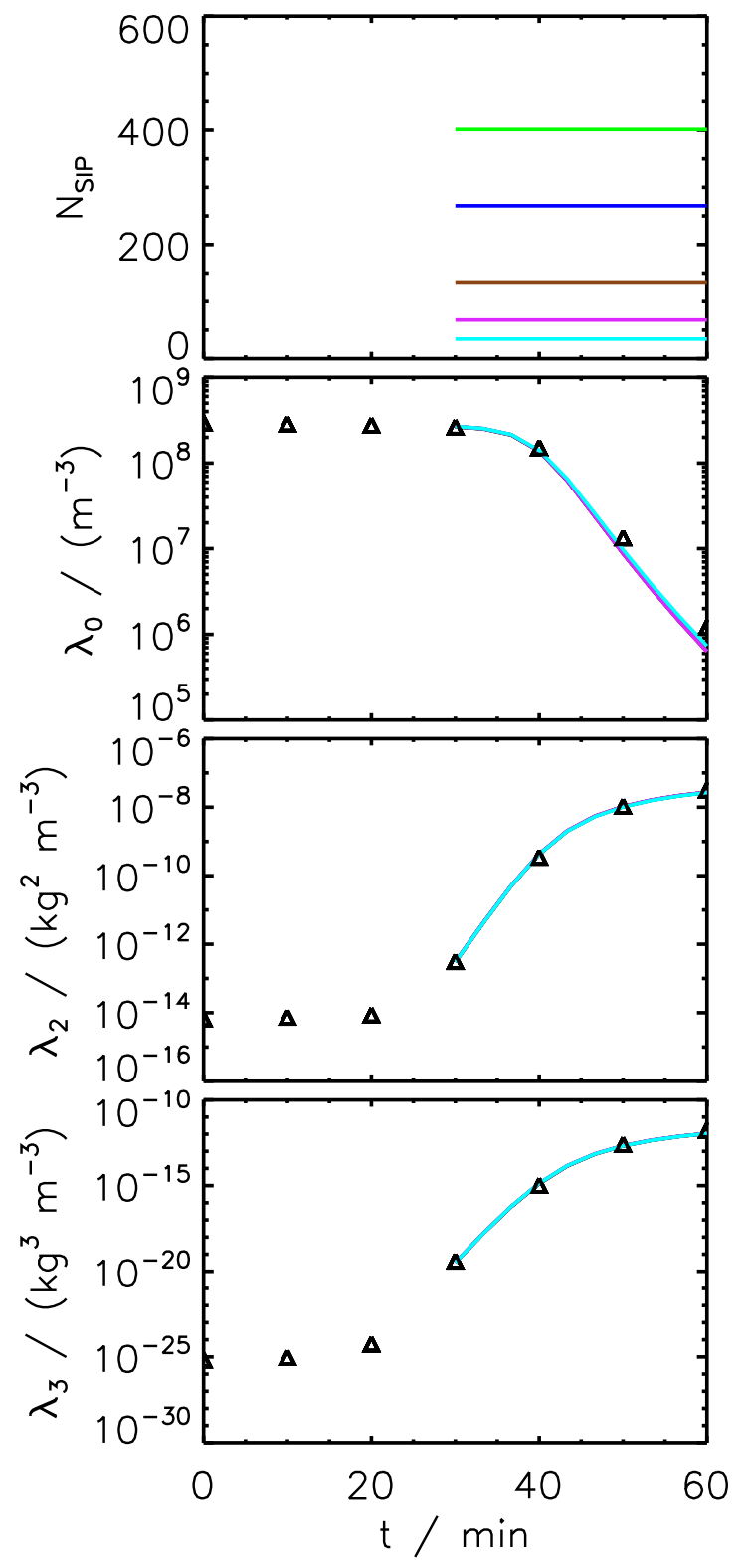

Figure 19: LONG AIM: Variation of bin resolution $\kappa$ at $t_{\text {init }}=30 \mathrm{~min}$. 
3.2.2 Long Kernel (AIM), $\nu_{\text {const }}$-init

3.2.2.1 Variation of $d t$ and $N_{S I P}$
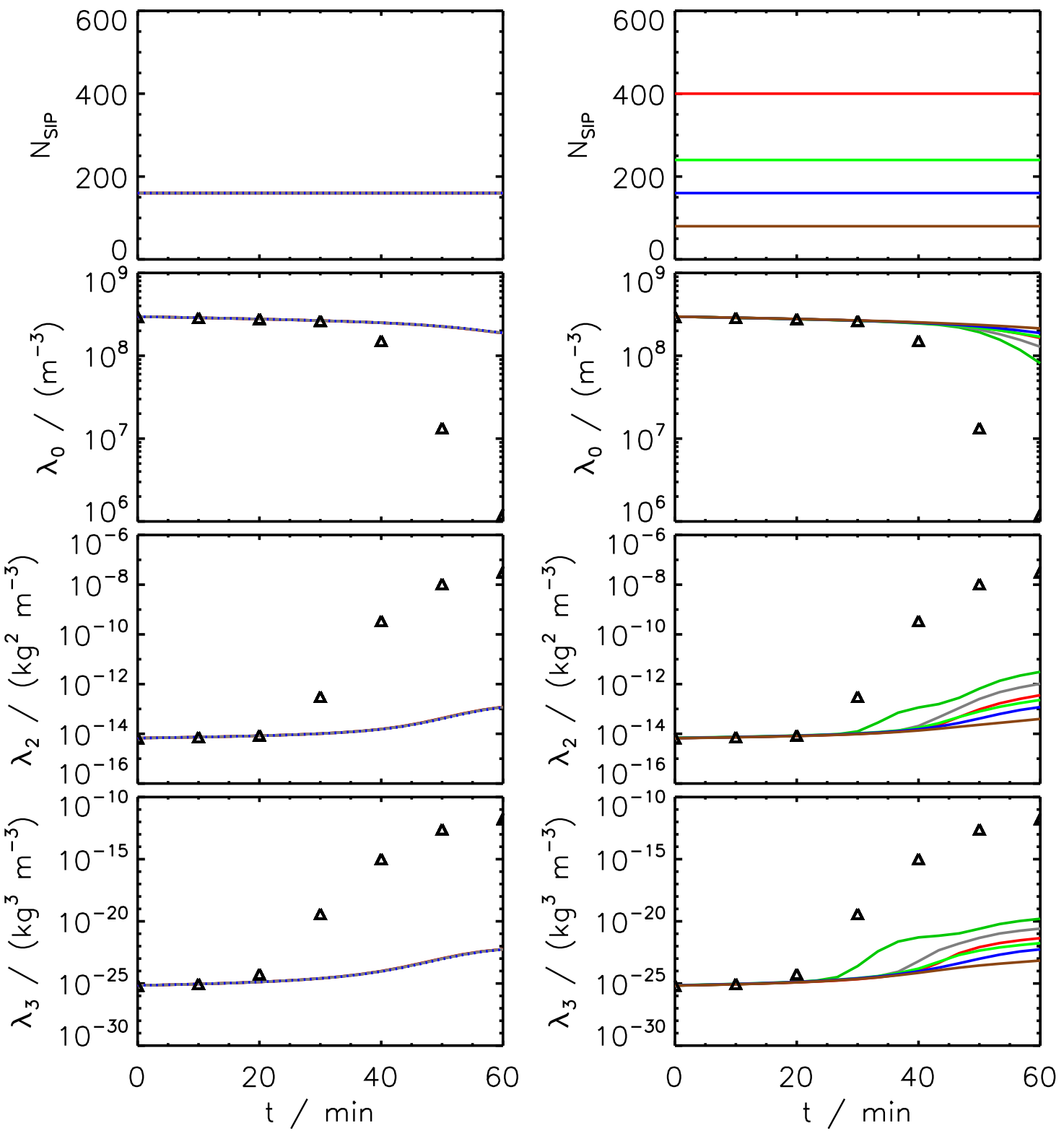

Figure 20: LONG AIM: Variation of time step $d t$ and SIP number $N_{S I P}$. 


\subsubsection{Long Kernel (AIM), $\nu_{d r a w}$-init}

\subsubsection{Variation of $d t$ and $N_{S I P}$}
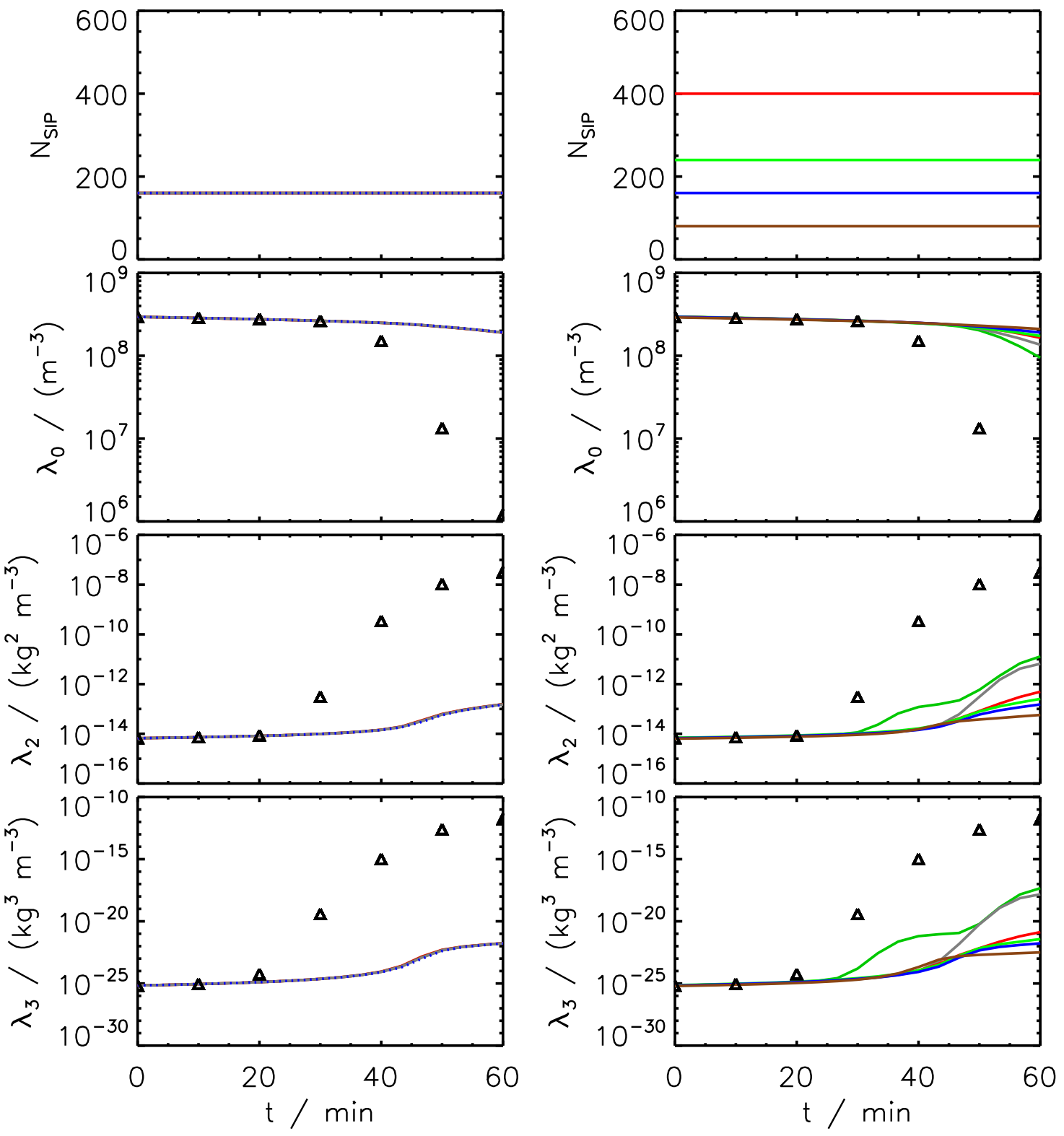

Figure 21: LONG AIM: Variation of time step $d t$ and SIP number $N_{S I P}$. 
3.2.4 Long Kernel (AIM), $\nu_{\text {random, } r s}$-init

3.2.4.1 Variation of $d t$ and $\alpha_{\text {low }}$
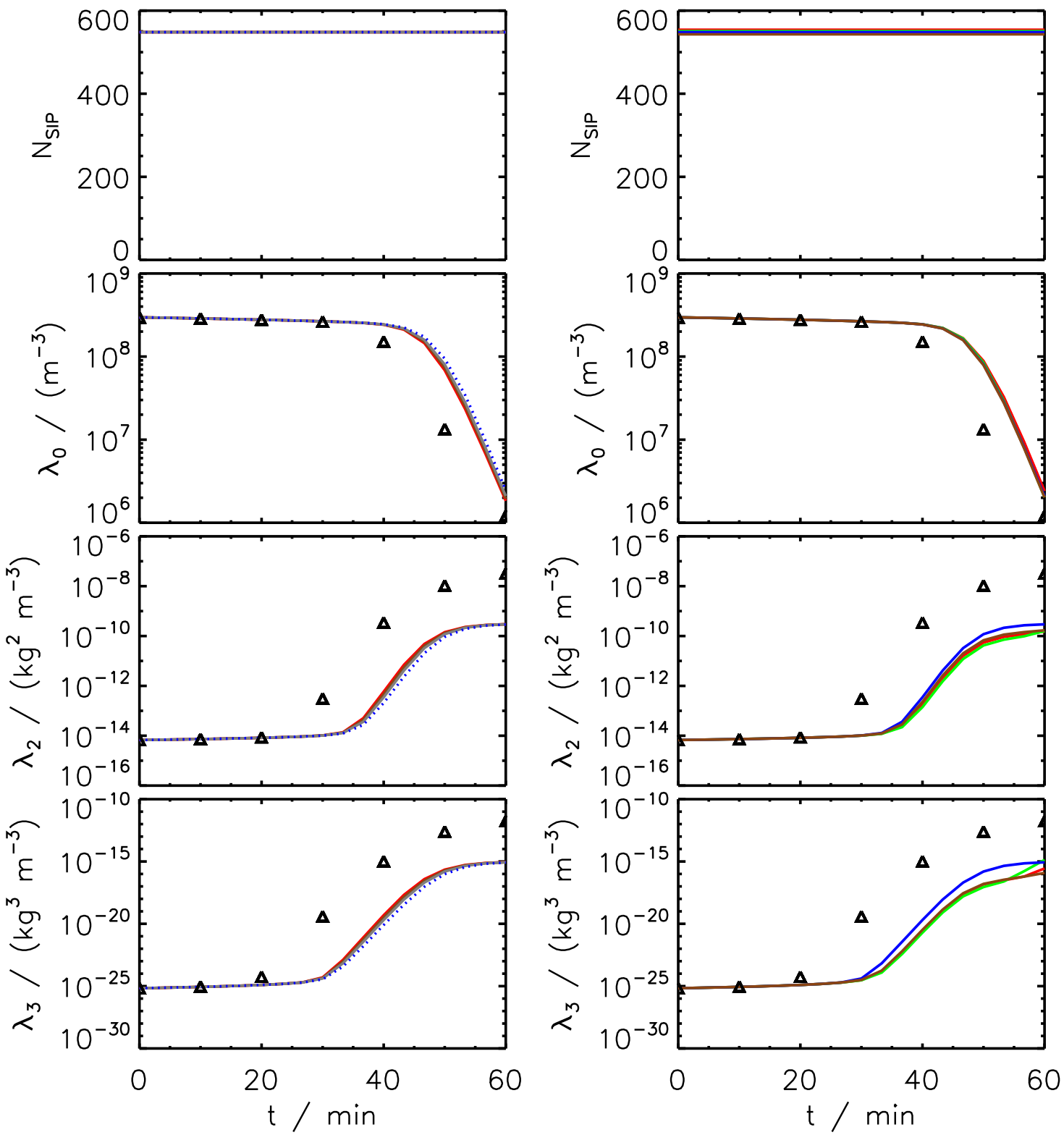

Figure 22: LONG AIM: Variation of time step $d t$ (left) and lower threshold $\alpha_{\text {low }}$ (right). 


\subsubsection{Long Kernel (AIM), $\nu_{\text {random }, l b}$-init}

\subsubsection{Variation of $d t$ and $\alpha_{\text {low }}$}
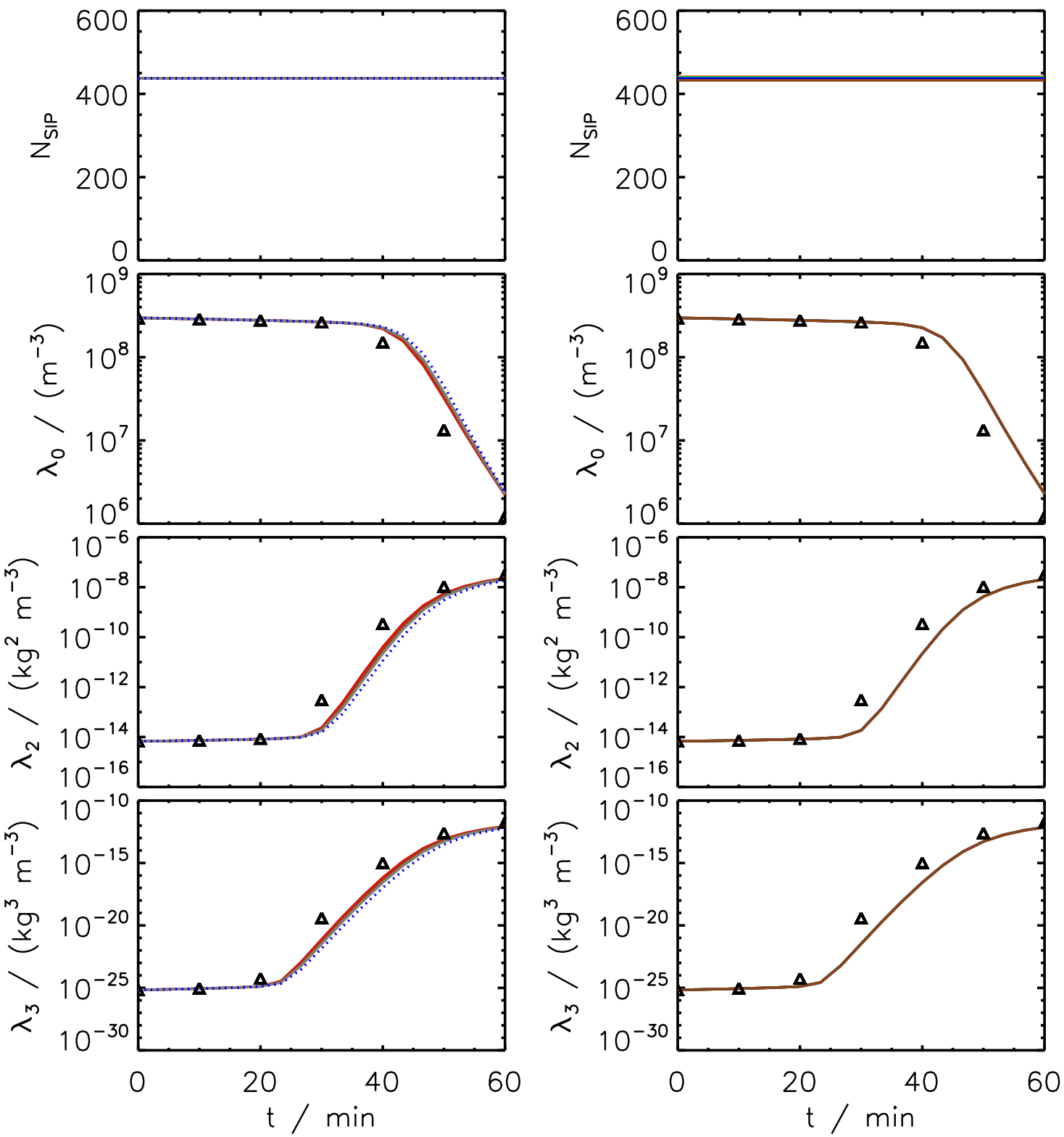

Figure 23: LONG AIM: Variation of time step $d t$ (left) and lower threshold $\alpha_{\text {low }}$ (right). 


\subsection{Hall Kernel (AIM)}

The following section show Hall AIM results for the SingleSIP init method. 


\subsubsection{Hall Kernel (AIM), SingleSIP-init}

\subsubsection{Variation of $d t$ and $\kappa$}
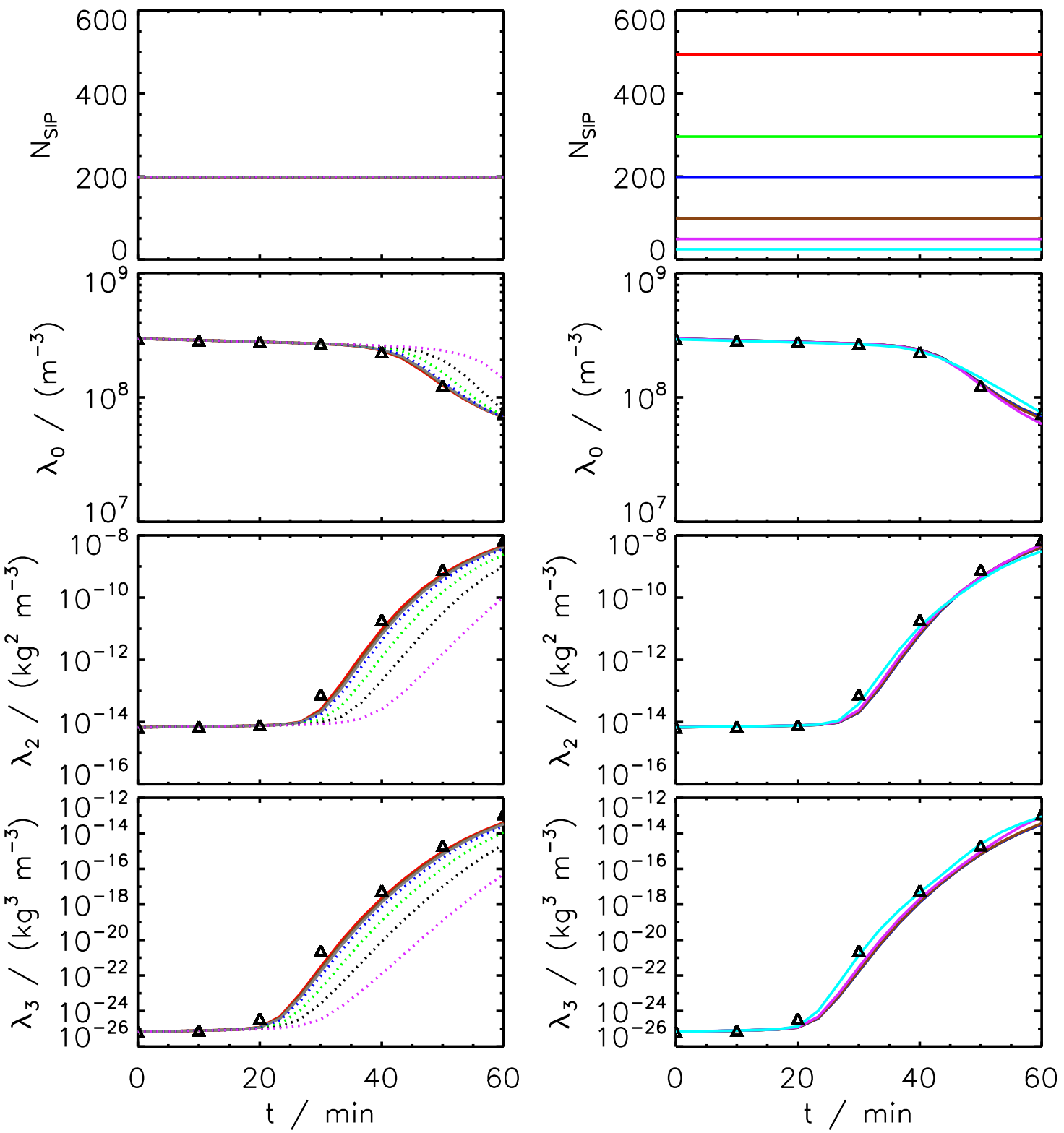

Figure 24: HALL AIM: Variation of time step $d t$ (left) and bin resolution $\kappa$ (right). 


\section{Simulation Results of All-or-Nothing Algorithm (AON)}

Block 4 displays AON simulation results for the Golovin, Long and Hall kernel. Additionally, simulations with a "constant efficiency" kernel are included.

All AON simulations use as default $d t=10 \mathrm{~s}$.

The SingleSIP initialisation method uses as default $\kappa=40, r_{\text {critmin }}=0.6 \mu \mathrm{m}$ and $\eta=10^{-8}$ and is probabilistic.

The $\nu_{\text {const }}$ and $\nu_{\text {draw }}$ initialisation methods use as default $N_{S I P}=160$. For equal-weights collections (basically occurs only if $\nu_{\text {const }}$ init is used), equal splitting $(\xi=0.5)$ is applied as default. 


\subsection{Golovin Kernel (AON)}

The following sections show Golovin AON results for three types of init methods and two variants of the SingleSIP-init method (change of $r_{\text {critmin }}$ and deterministic version). 


\subsubsection{Golovin Kernel (AON), SingleSIP-init}

\subsubsection{Variation of $d t$ and $\kappa$}
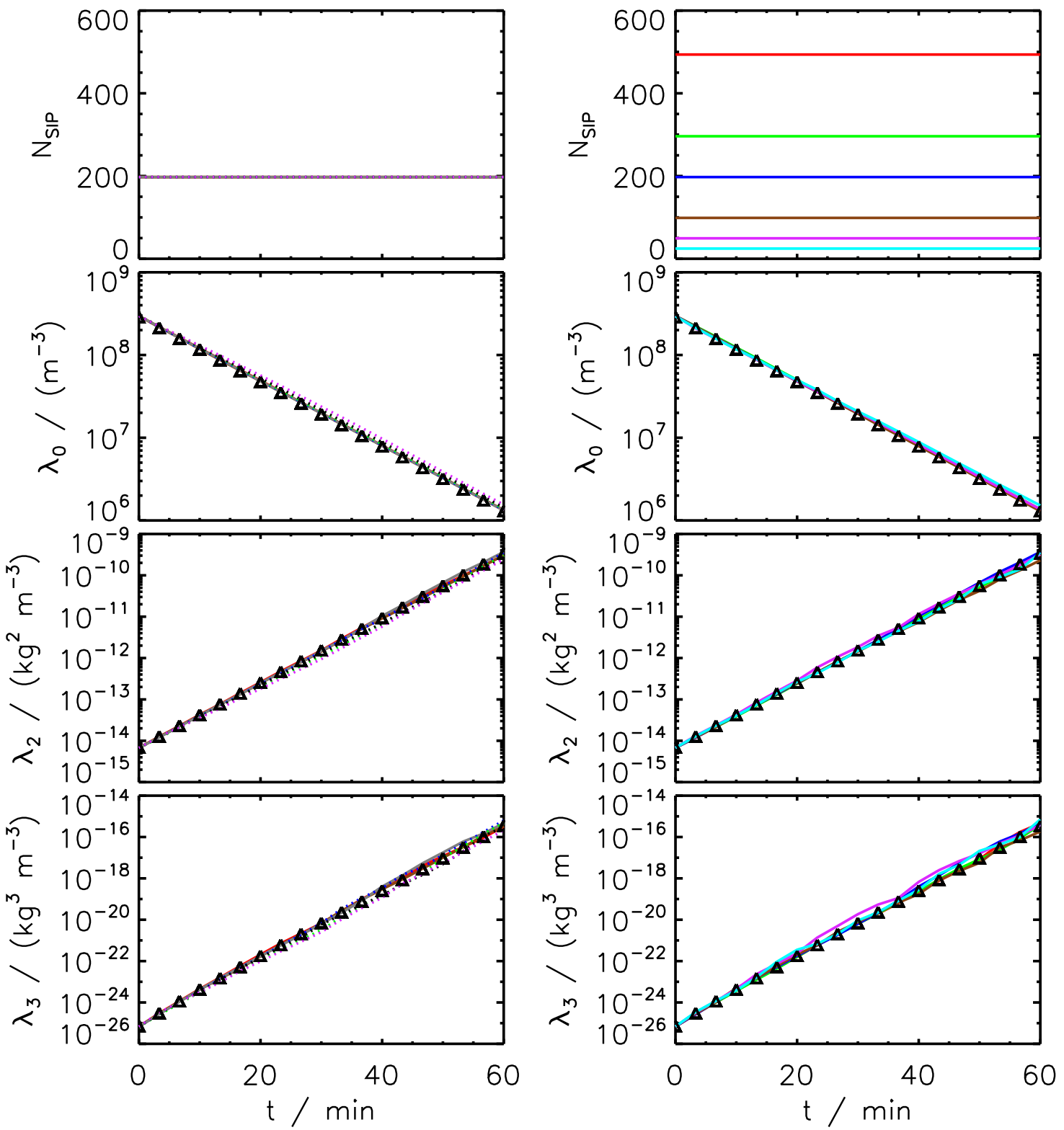

Figure 25: GOL AON: Variation of time step $d t$ (left) and bin resolution $\kappa$ (right). 
4.1.2 Golovin Kernel (AON), SingleSIP-init with $r_{\text {critmin }}=1.6 \mu \mathrm{m}$

\subsubsection{Variation of $d t$ and $\kappa$}
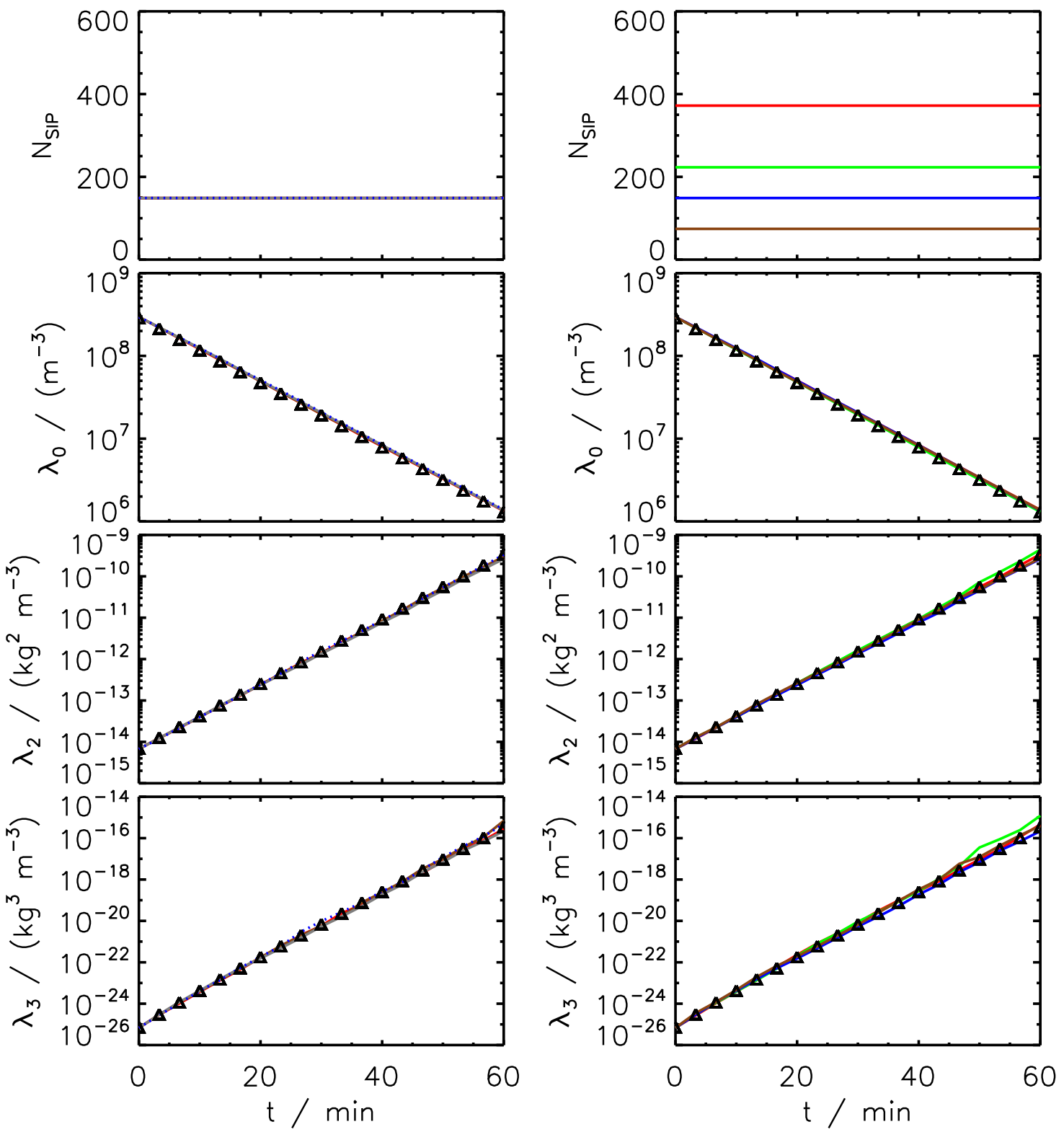

Figure 26: GOL AON: Variation of time step $d t$ (left) and bin resolution $\kappa$ (right). 


\subsubsection{Golovin Kernel (AON), Deterministic SingleSIP-init}

\subsubsection{Variation of $d t$ and $\kappa$}
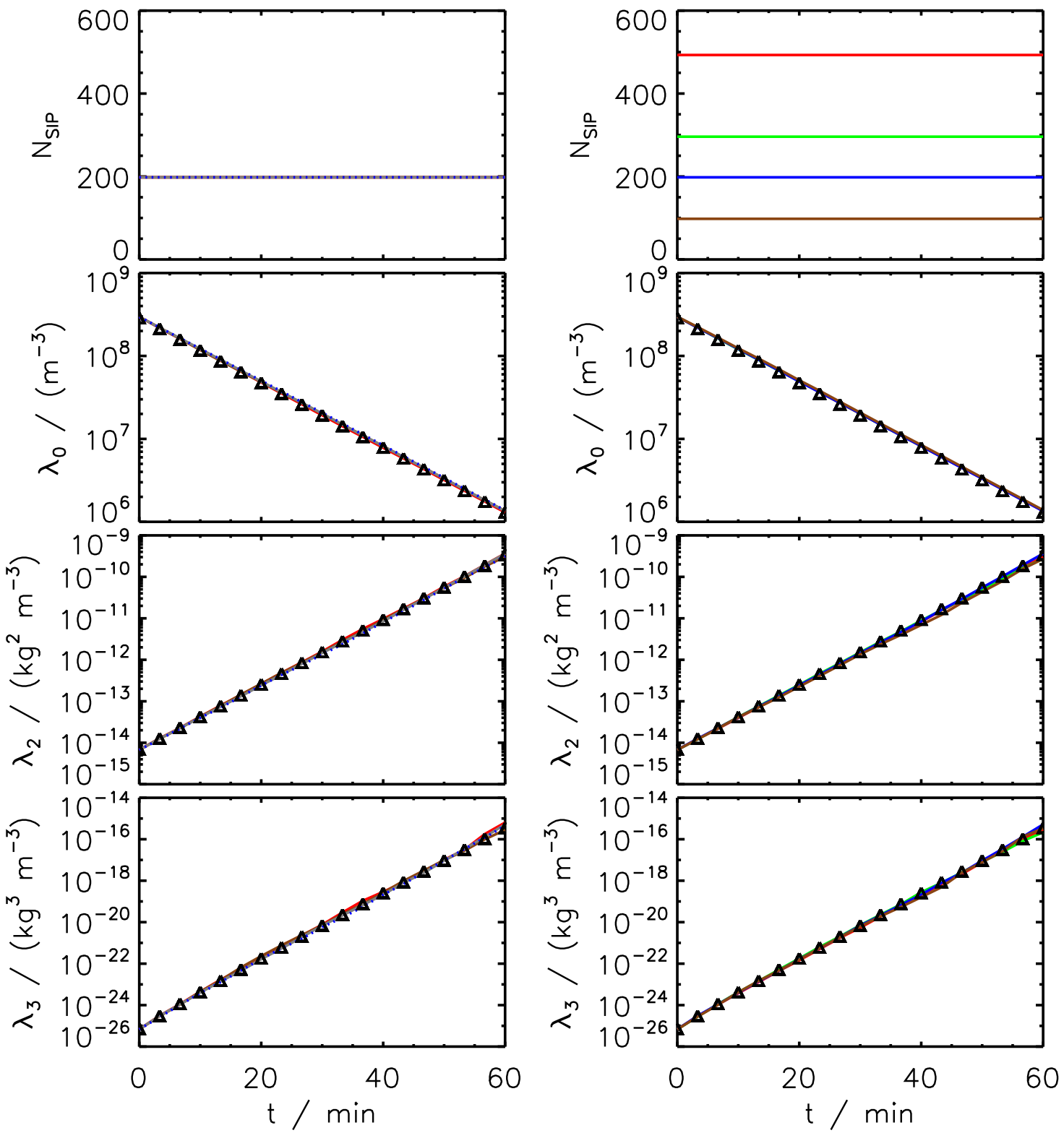

Figure 27: GOL AON: Variation of time step $d t$ (left) and bin resolution $\kappa$ (right). 


\subsubsection{Golovin Kernel (AON), $\nu_{\text {const }}$-init}

\subsubsection{Variation of $d t$ and $N_{S I P}$}
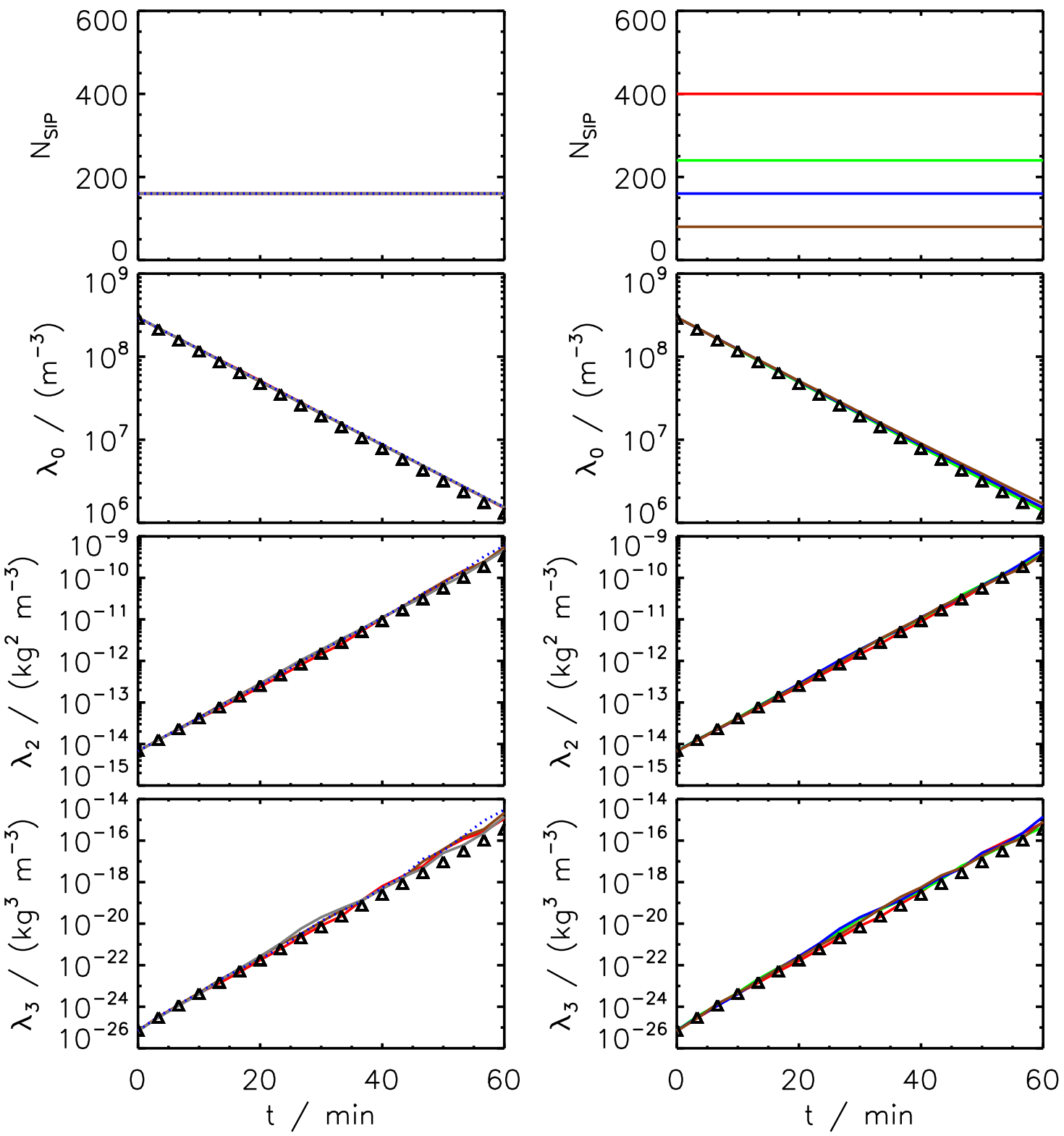

Figure 28: GOL AON: Variation of time step $d t$ and SIP number $N_{S I P}$. 


\subsubsection{Golovin Kernel (AON), $\nu_{d r a w}$-init}

\subsubsection{Variation of $d t$ and $N_{S I P}$}
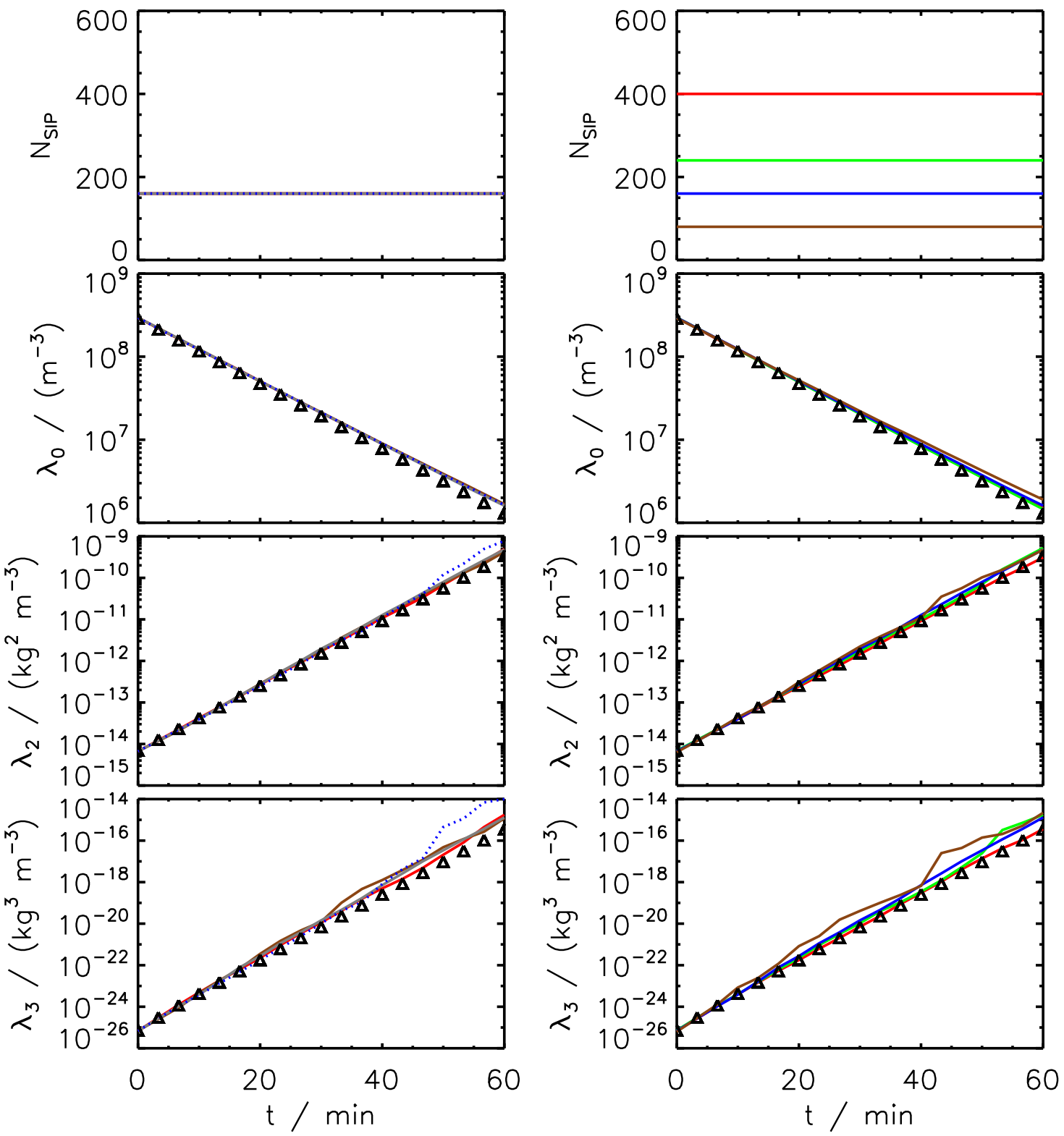

Figure 29: GOL AON: Variation of time step $d t$ and SIP number $N_{S I P}$. 


\subsection{Long Kernel (AON)}

The following sections show Long AON results for six types of init methods. 


\subsubsection{Long Kernel (AON), SingleSIP-init}

\subsubsection{Variation of $d t$ and $\kappa$}
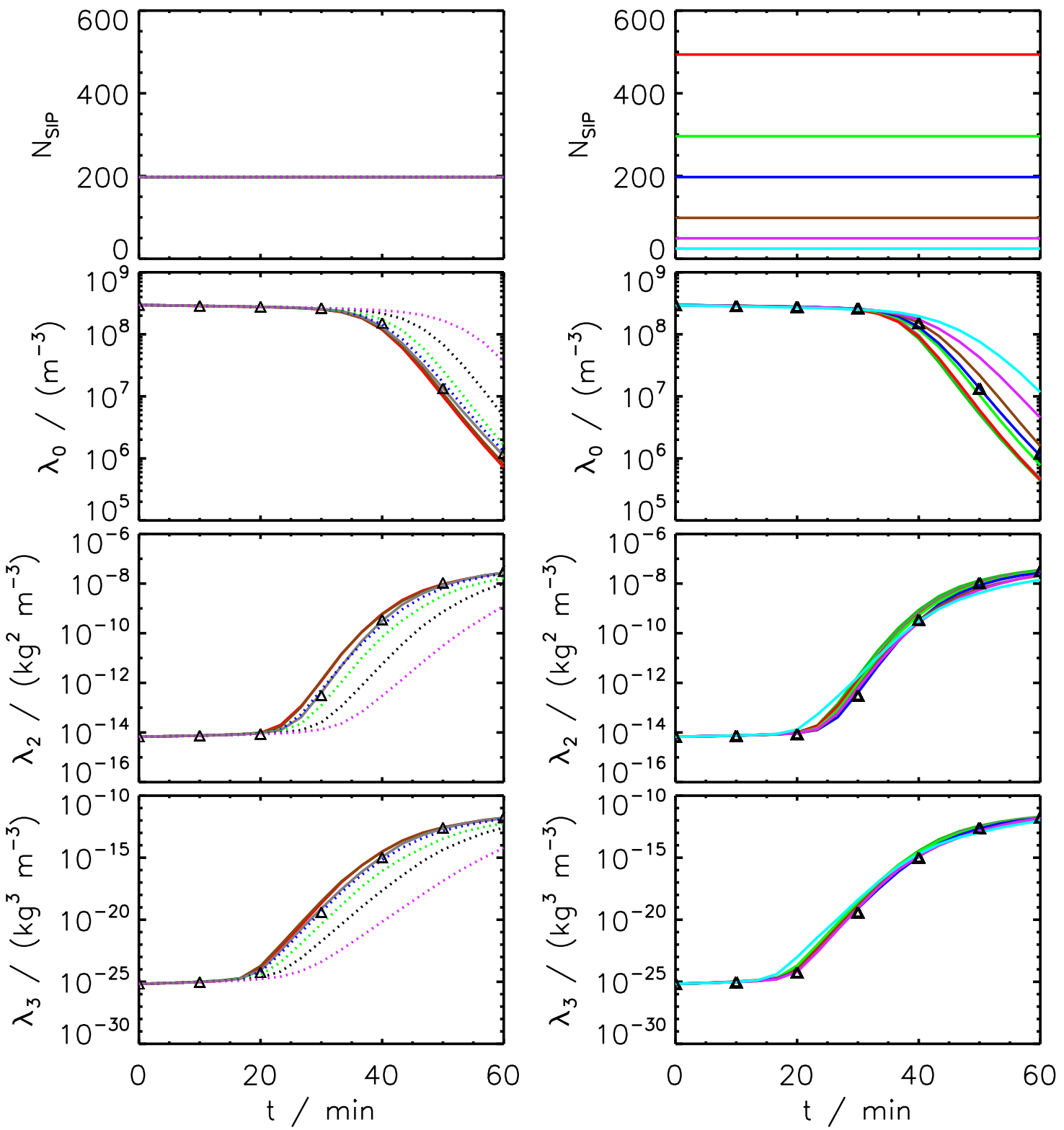

Figure 30: LONG AON: Variation of time step $d t$ (left) and bin resolution $\kappa$ (right). 


\subsubsection{Variation of $\eta$}

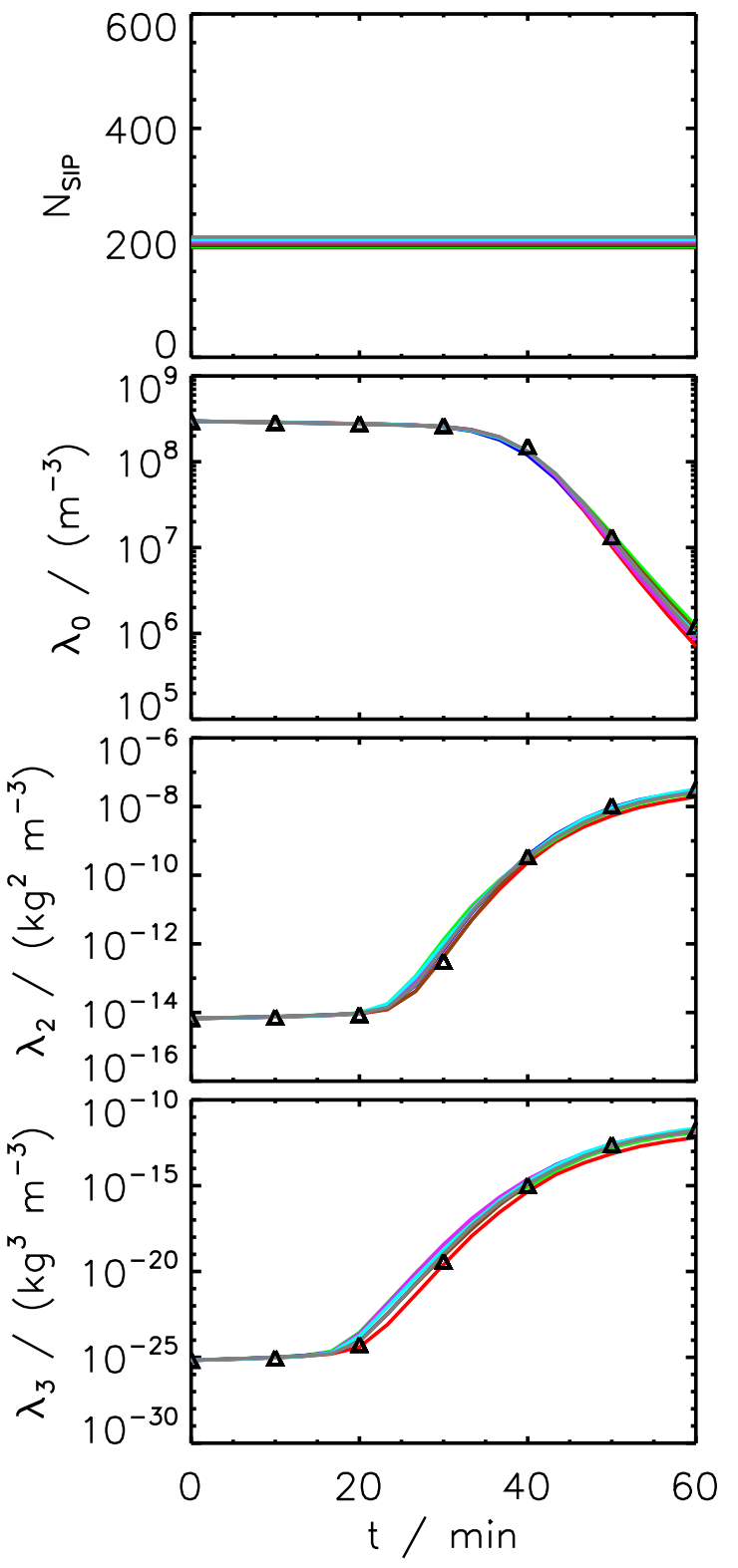

Figure 31: LONG AON: Variation of threshold $\eta$. 


\subsubsection{No multiple collections}

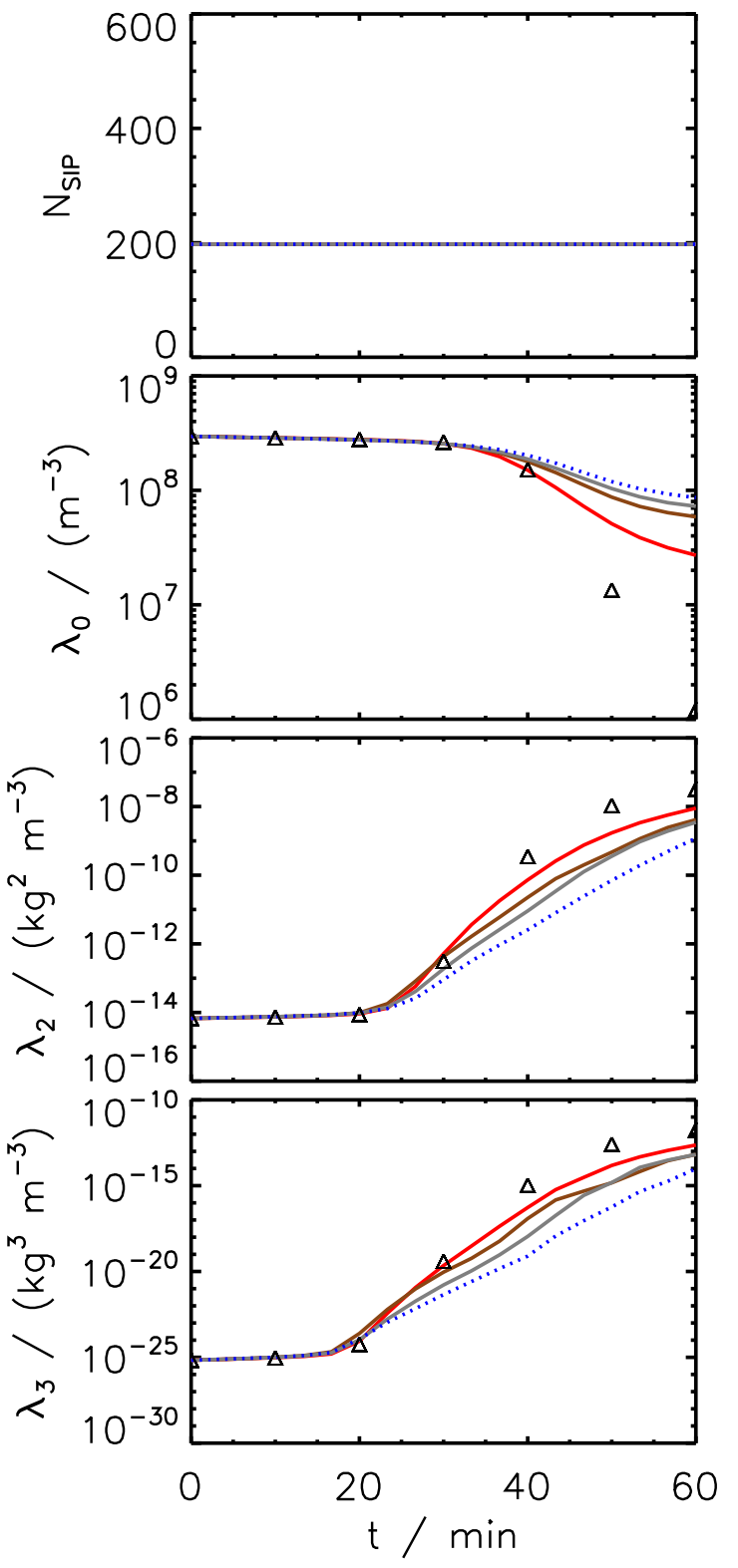

Figure 32: LONG AON: Variation of time step $d t$. 


\subsubsection{Variation of $r_{\text {critmin }}$}
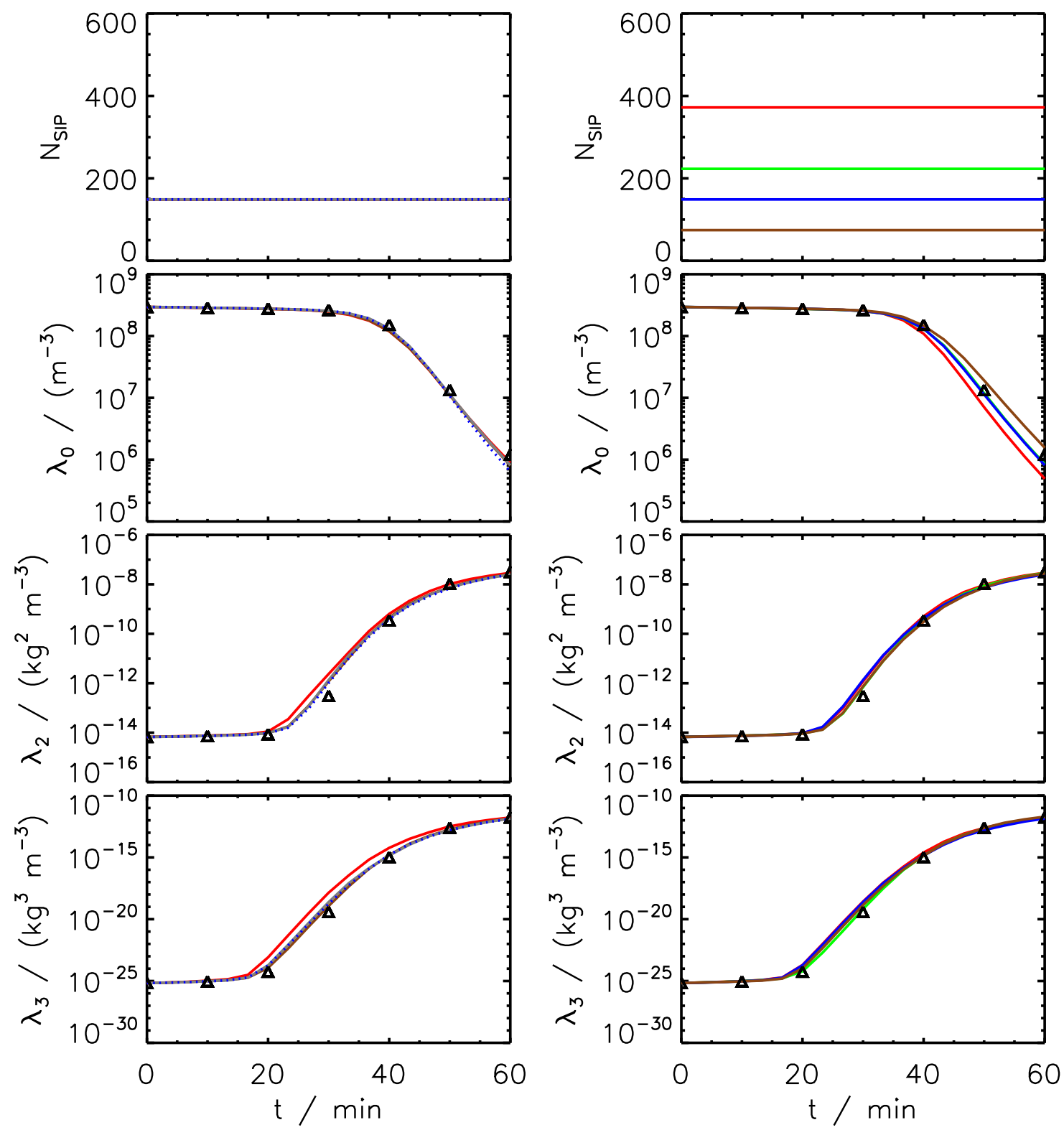

Figure 33: LONG AON: Variation of time step $d t$ (left) and bin resolution $\kappa$ (right) at $r_{\text {critmin }}=1.6 \mu \mathrm{m}$. 

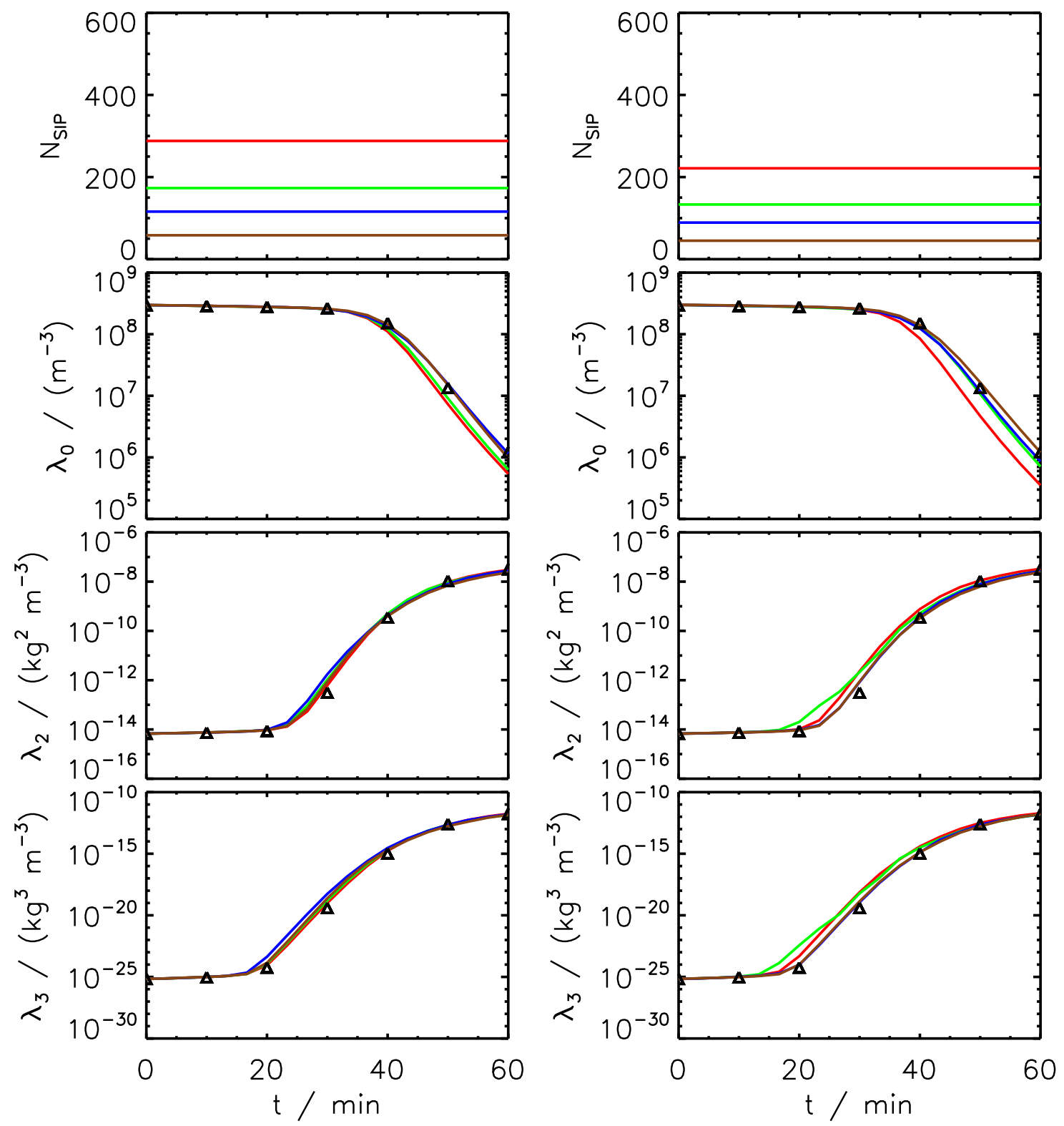

Figure 34: LONG AON: Variation of bin resolution $\kappa$ at $r_{\text {critmin }}=3.0 \mu \mathrm{m}$ (left) and $5.0 \mu \mathrm{m}$ (right). 


\subsubsection{Variation of $t_{\text {init }}$}
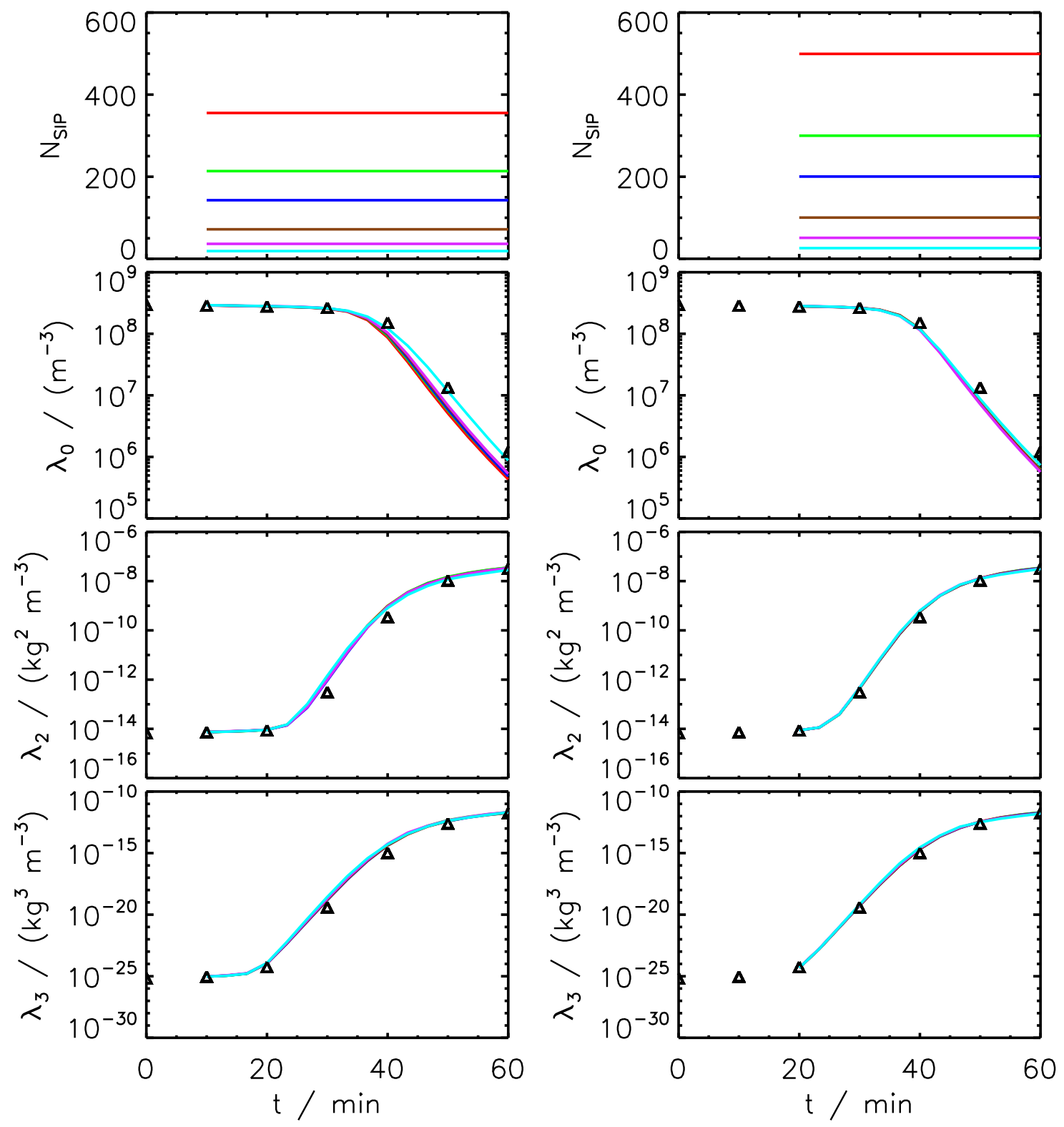

Figure 35: LONG AON: Variation of bin resolution $\kappa$ at $t_{\text {init }}=10 \mathrm{~min}$ (left) and $20 \mathrm{~min}$ (right). 


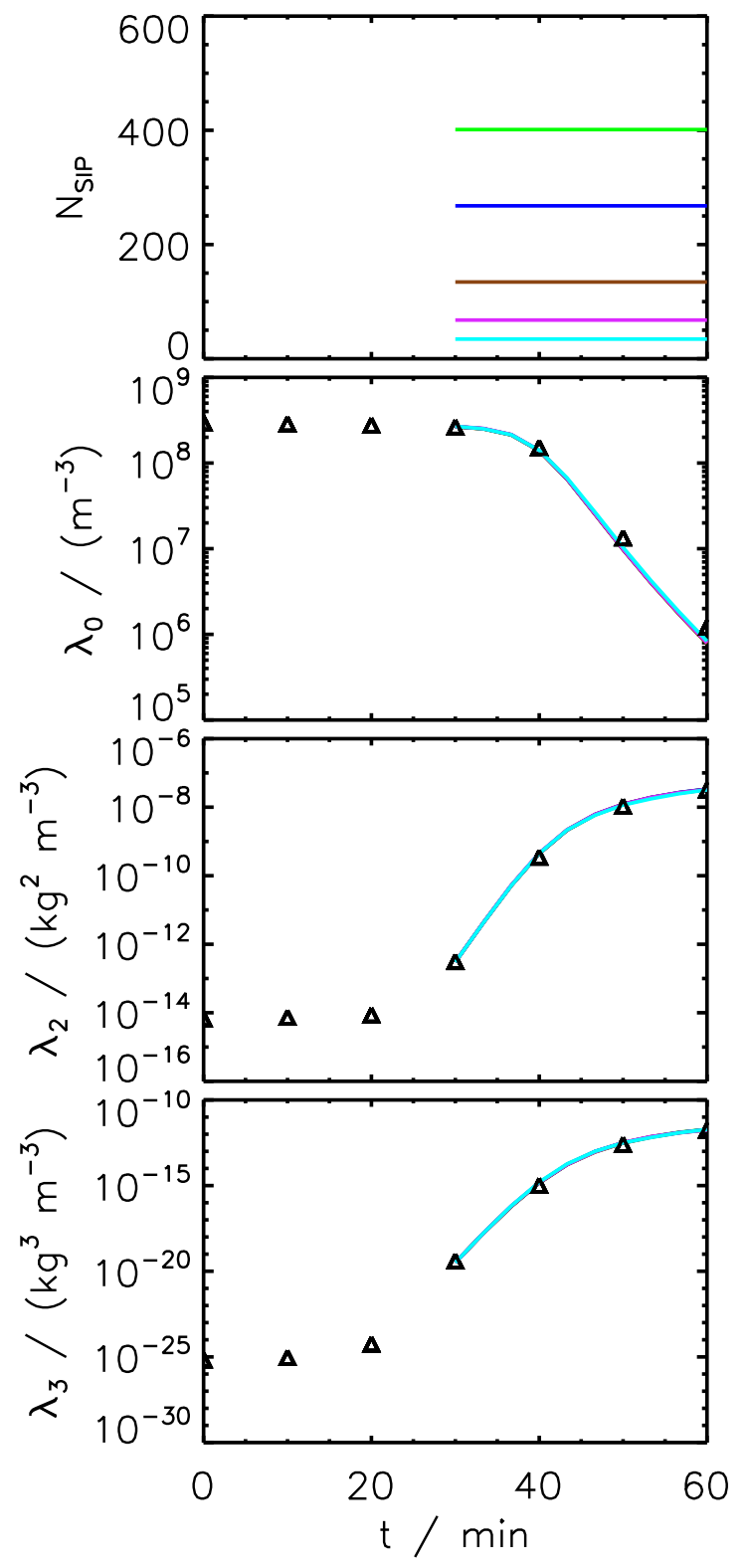

Figure 36: LONG AON: Variation of bin resolution $\kappa$ at $t_{\text {init }}=30 \mathrm{~min}$. 
4.2.1.6 Order of combination processing The AON algorithm is inherently updating the SIP weights on the fly. Then, the results may depend on the order the SIP combinations are processed. As default, the algorithm starts with combinations of the smallest SIPs. The version $\mathrm{OTF}_{l}$ first treats the largest SIPs, the RANDorder version uses in each time iteration a randomly chosen order.
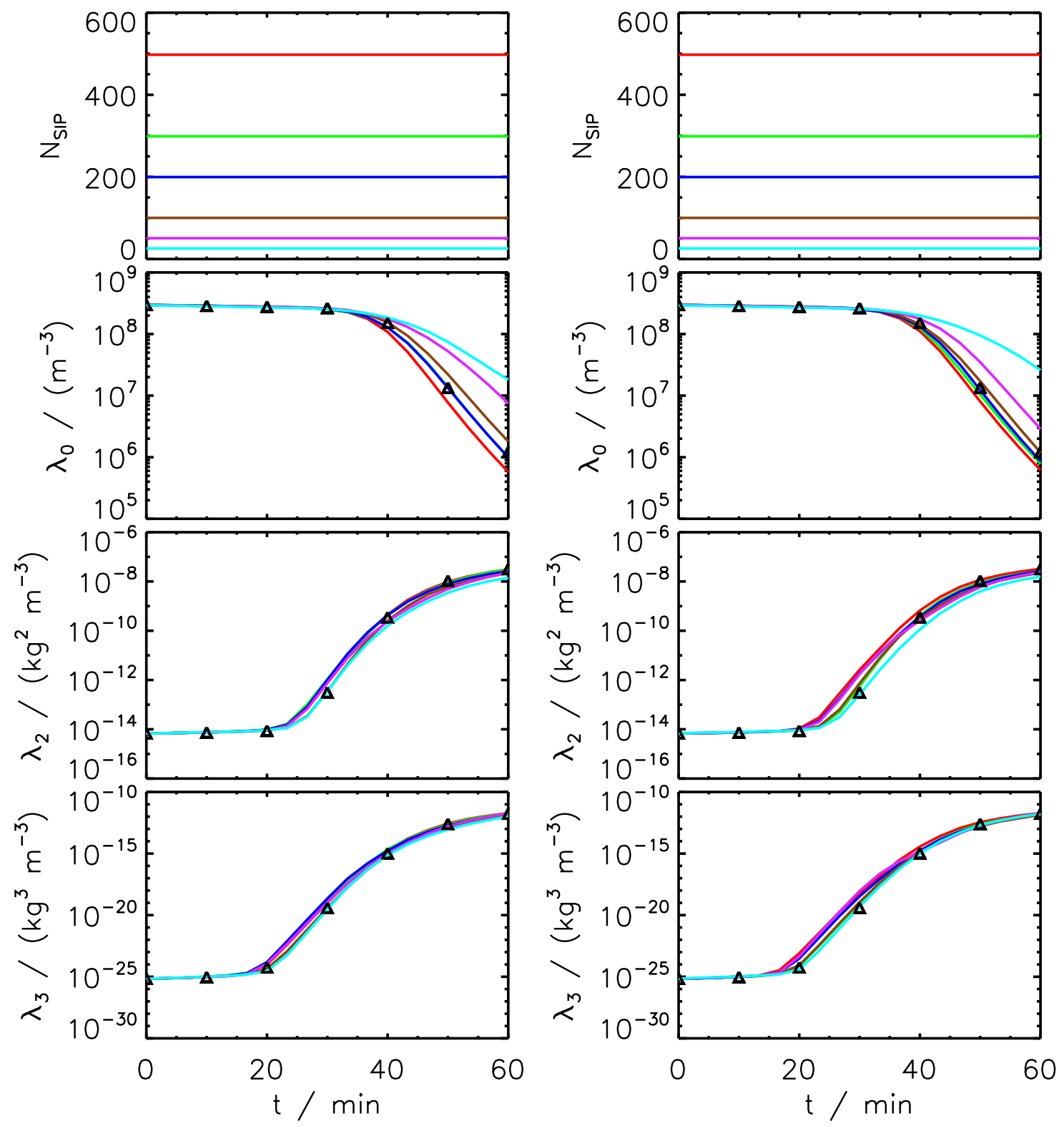

Figure 37: LONG AON: Variation of bin resolution $\kappa \mathrm{OTF}_{l}$ (left) and RANDorder (right) 
4.2.1.7 Hybrid init The radius spectrum is divided into two parts. For radii $r>16 \mu \mathrm{m}$, a high resolution with $\kappa_{\text {high }}=100$ is used irrespetive of the chosen $\kappa$. For smaller radii, the chosen $\kappa$ is used.

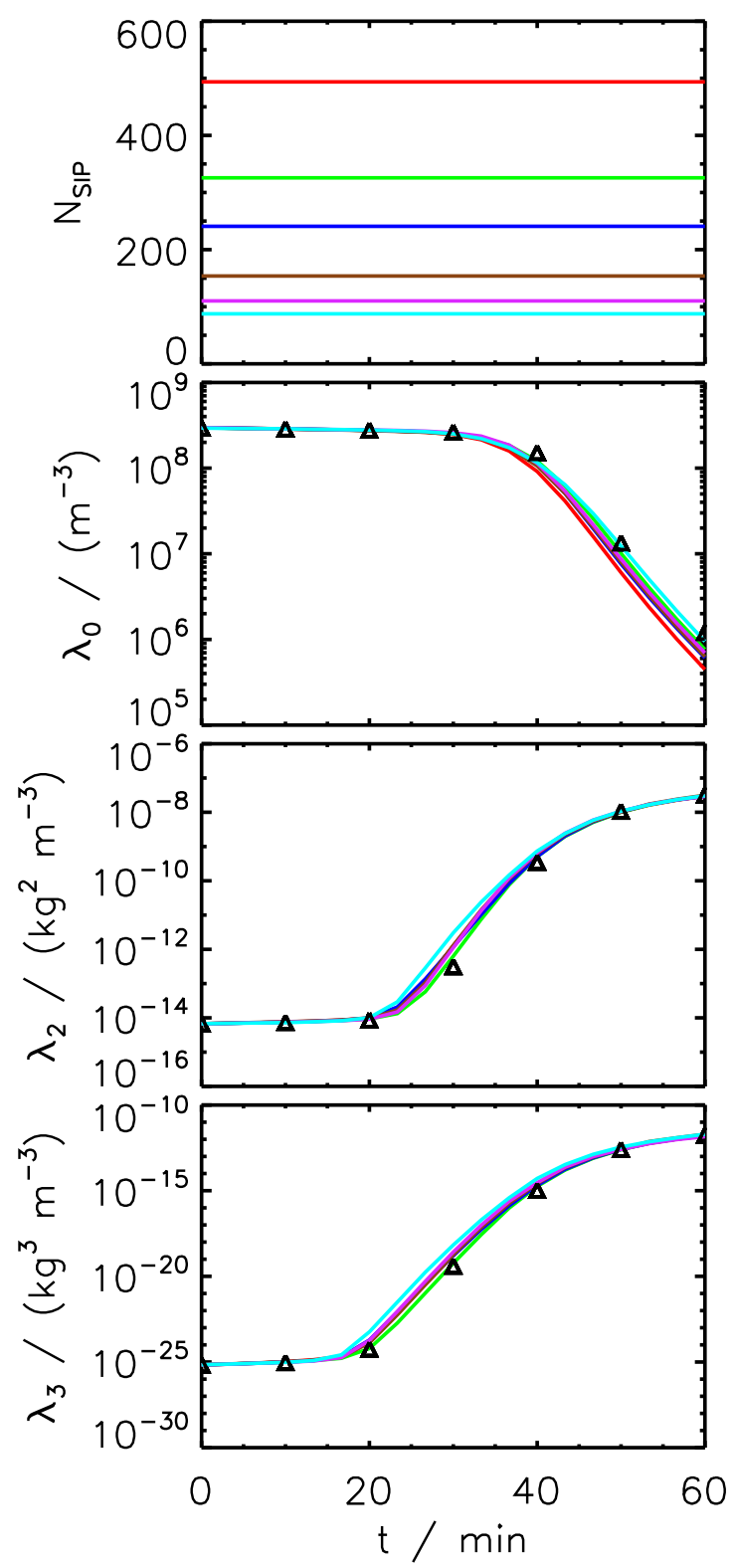

Figure 38: LONG AON: Variation of bin resolution $\kappa$ (for $r<16 \mu \mathrm{m}$ ). For $r>16 \mu \mathrm{m}$, $\kappa_{\text {high }}=100$ is used. 


\subsubsection{Long Kernel (AON), MultiSIP-init}
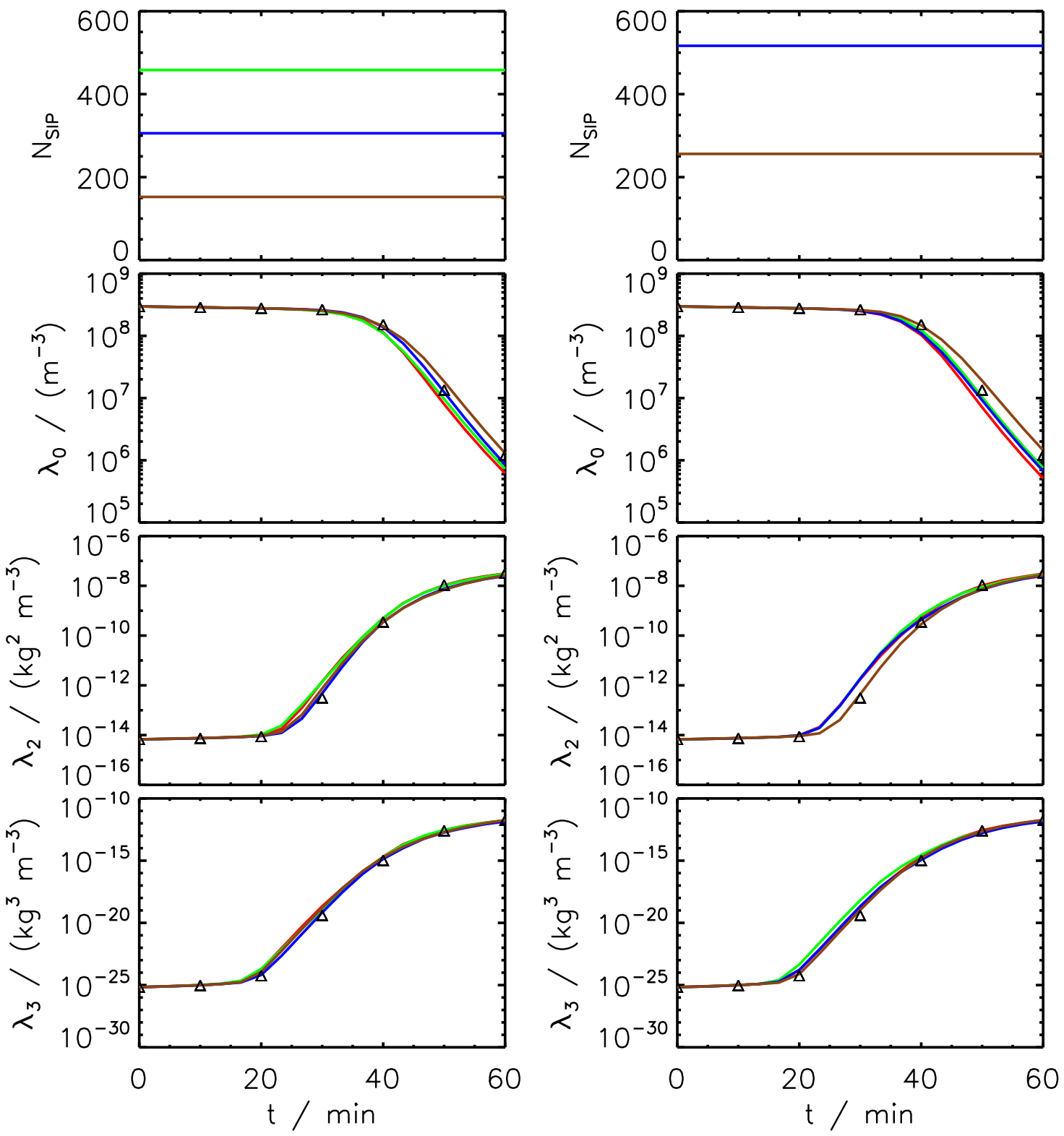

Figure 39: LONG AON: Variation of bin resolution $\kappa$ for $\nu_{\text {critmax }}=0.2 \cdot \max \nu_{b}$ (left) and $\nu_{\text {critmax }}=0.1 \cdot \max \nu_{b}$ (right). 


\subsubsection{Long Kernel (AON), $\nu_{\text {const }}$-init}

For collections with $\nu_{i}=\nu_{j}, \xi=0.5$.

\subsubsection{Variation of $d t$ and $N_{S I P}$}
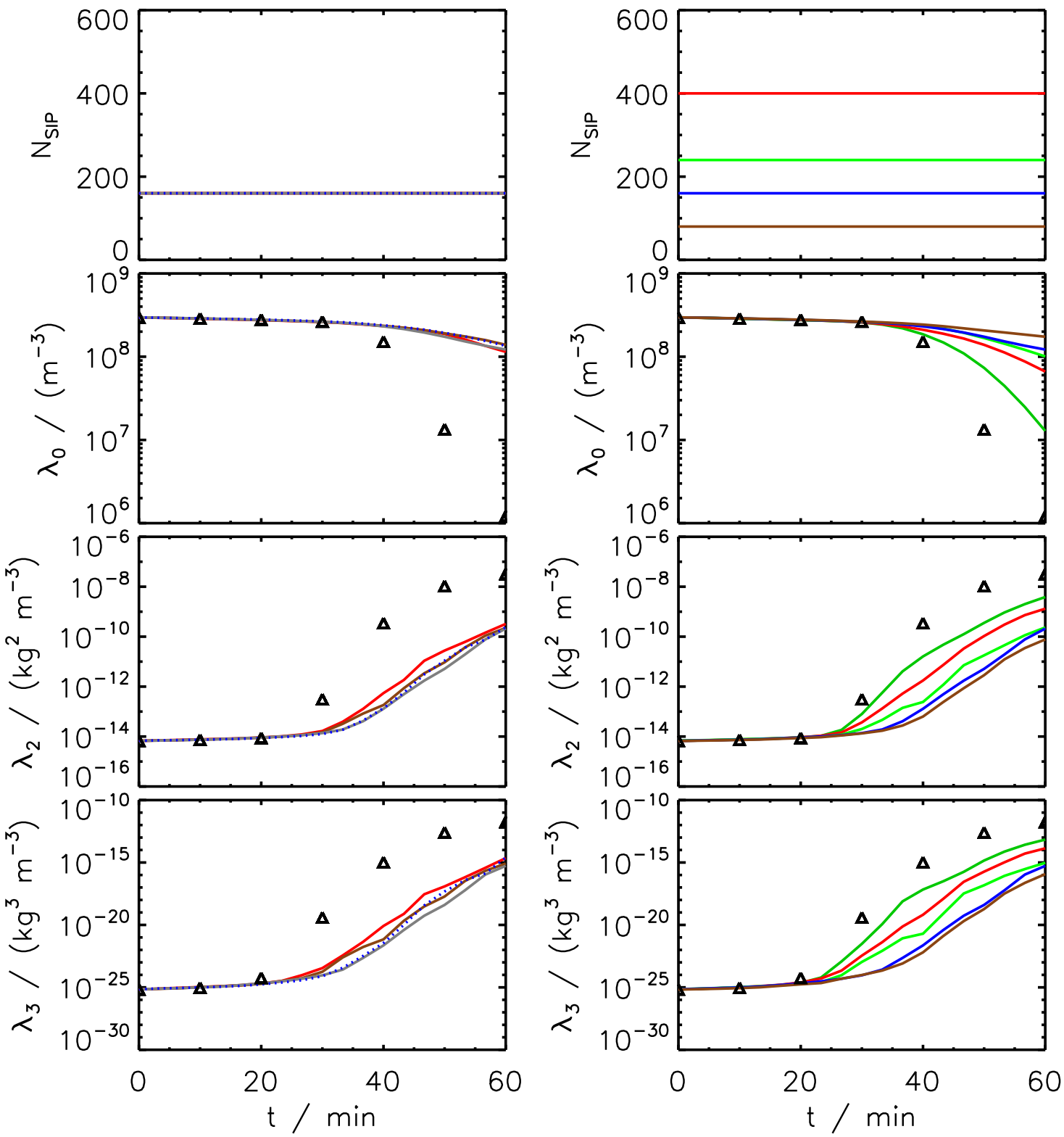

Figure 40: LONG AON: Variation of time step $d t$ and SIP number $N_{S I P}$. 


\subsubsection{Long Kernel (AON), $\nu_{\text {const }}$-init, randlin $\xi$}

For collections with $\nu_{i}=\nu_{j}, \xi=\operatorname{rand}()$, where rand() generates uniformly distributed random numbers from 0 to 1 .

\subsubsection{Variation of $d t$ and $N_{S I P}$}
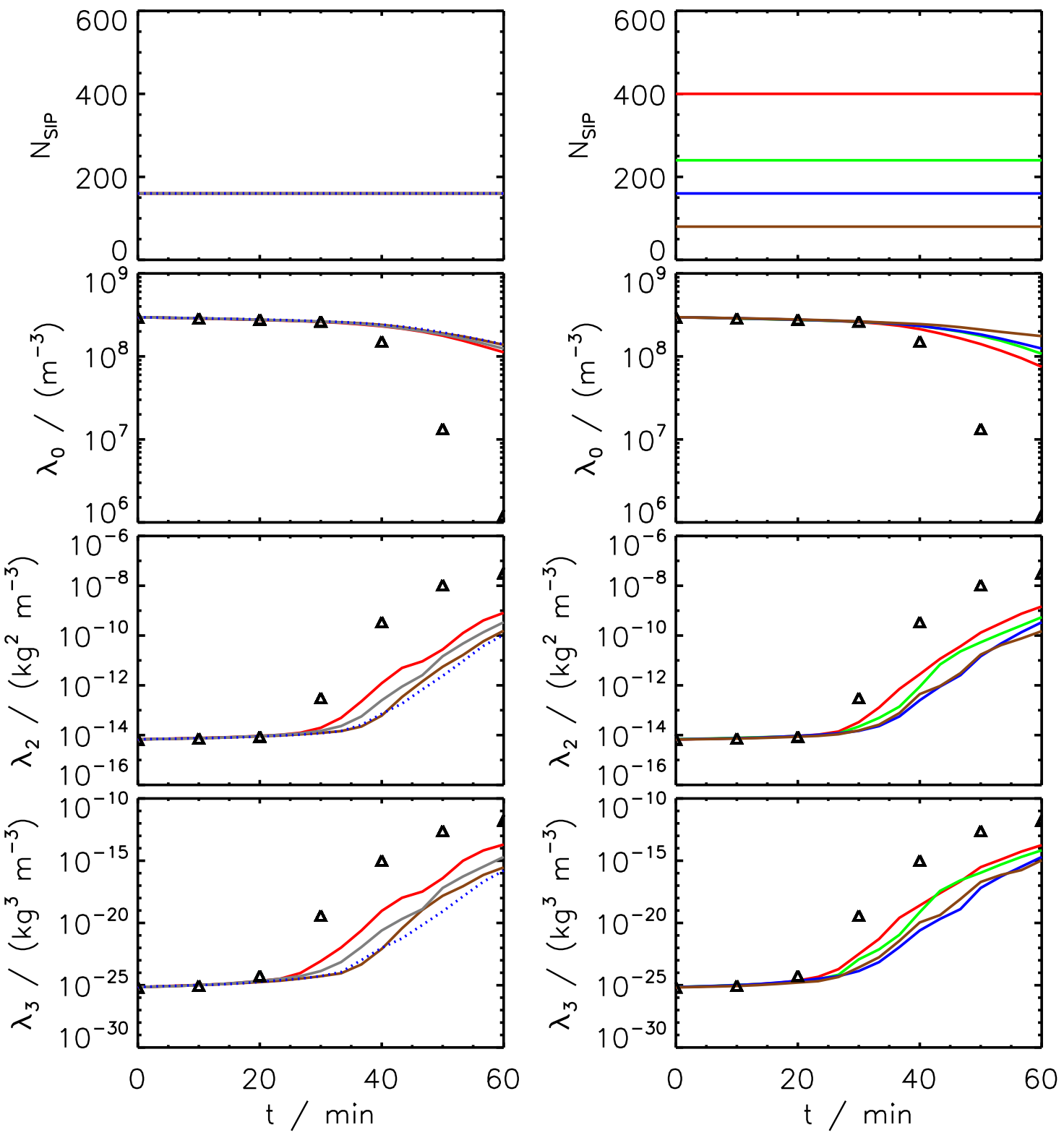

Figure 41: LONG AON: Variation of time step $d t$ and SIP number $N_{S I P}$. 


\subsubsection{Long Kernel (AON), $\nu_{\text {const }}$-init, randlog $\xi$}

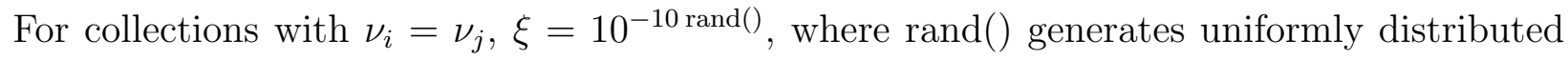
random numbers from 0 to 1 .

\subsubsection{Variation of $d t$ and $N_{S I P}$}
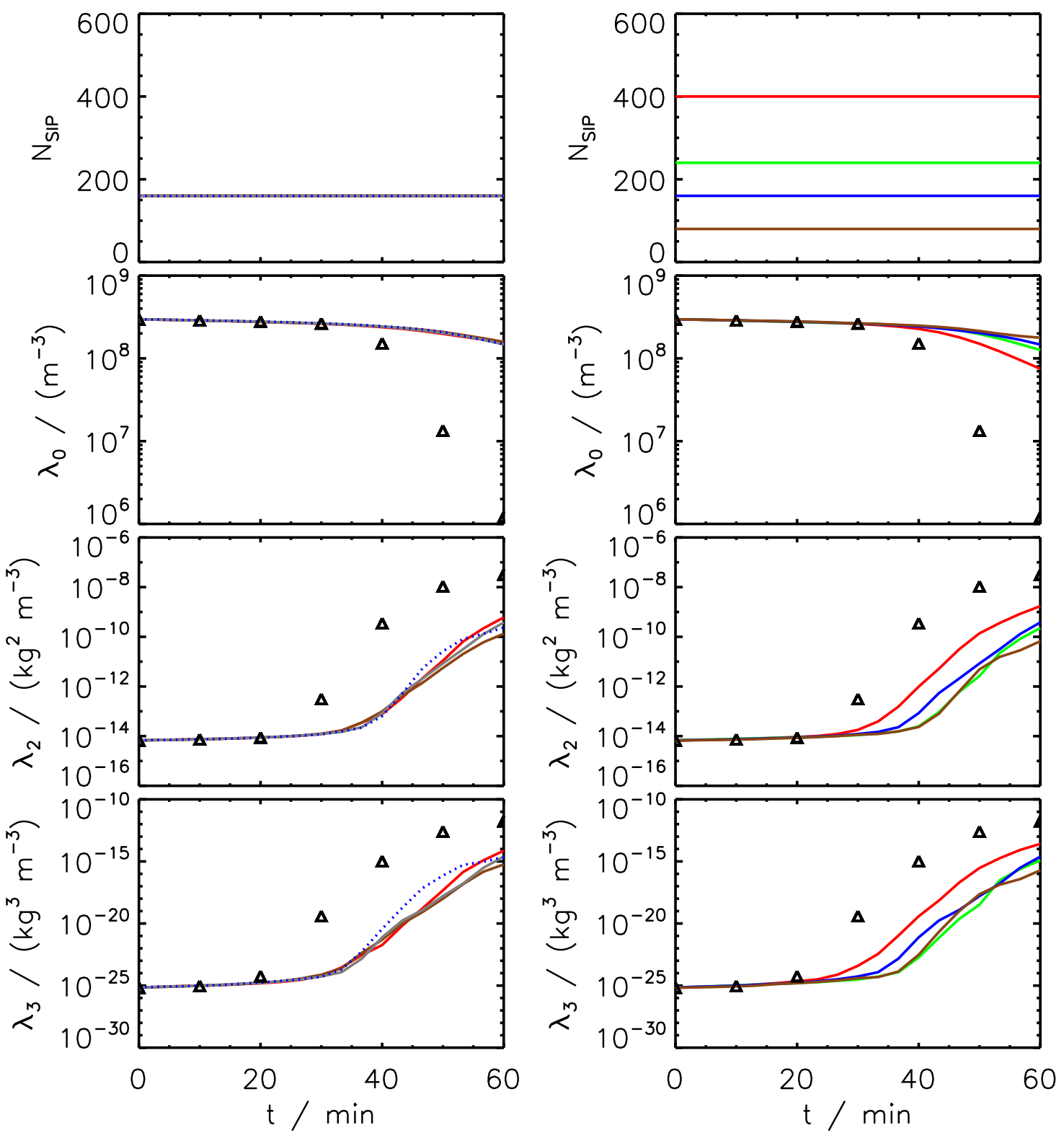

Figure 42: LONG AON: Variation of time step $d t$ and SIP number $N_{S I P}$. 


\subsubsection{Long Kernel (AON), $\nu_{\text {const }}$-init, randlog $\xi$ v2}

For collections with $\nu_{i}=\nu_{j}, \xi=10^{-10 \operatorname{rand}()^{2}}$, where rand() generates uniformly distributed random numbers from 0 to 1 .

\subsubsection{Variation of $d t$ and $N_{S I P}$}
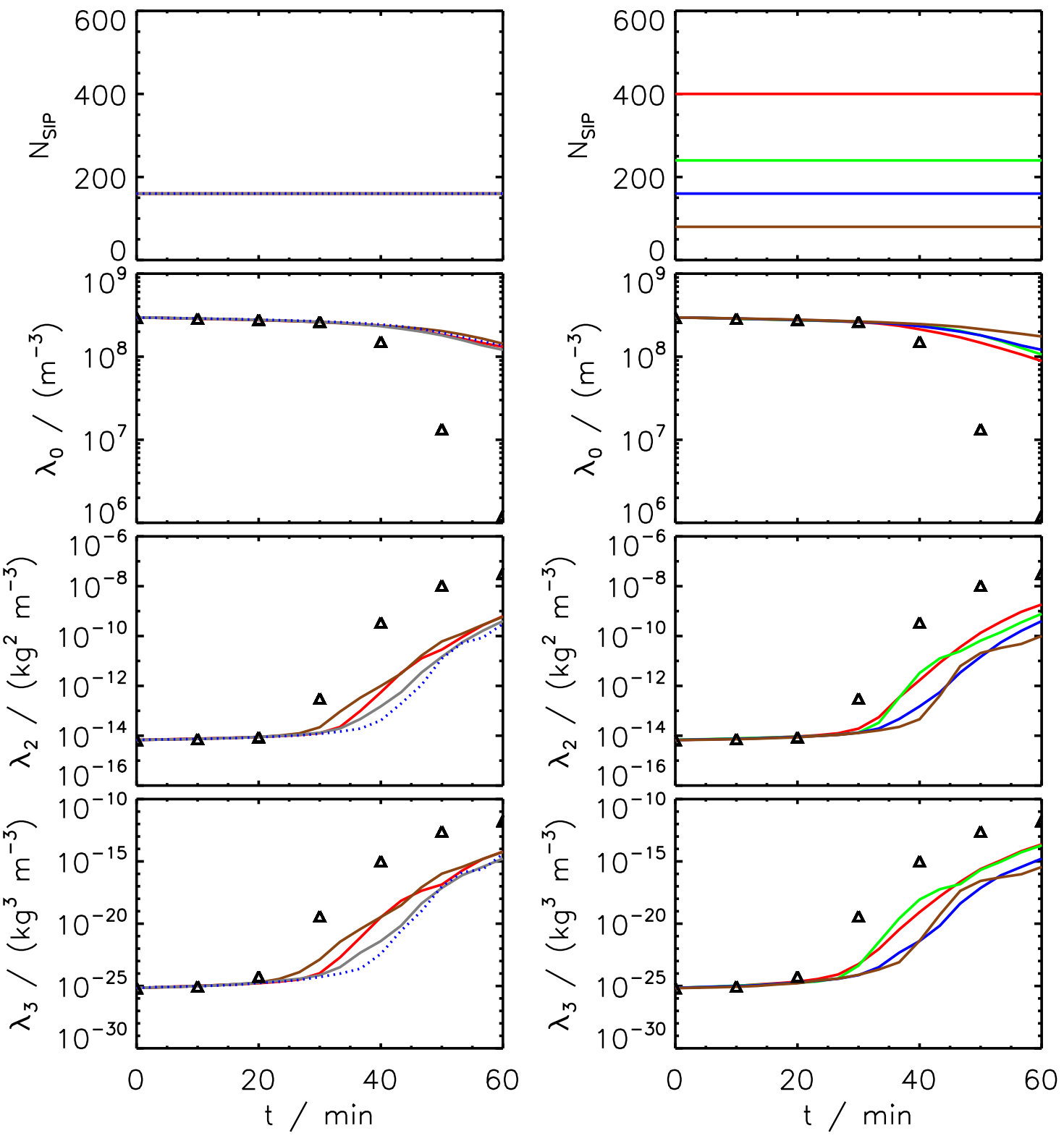

Figure 43: LONG AON: Variation of time step $d t$ and SIP number $N_{S I P}$. 
4.2.7 Long Kernel (AON), $\nu_{d r a w}$-init

4.2.7.1 Variation of $d t$ and $N_{S I P}$
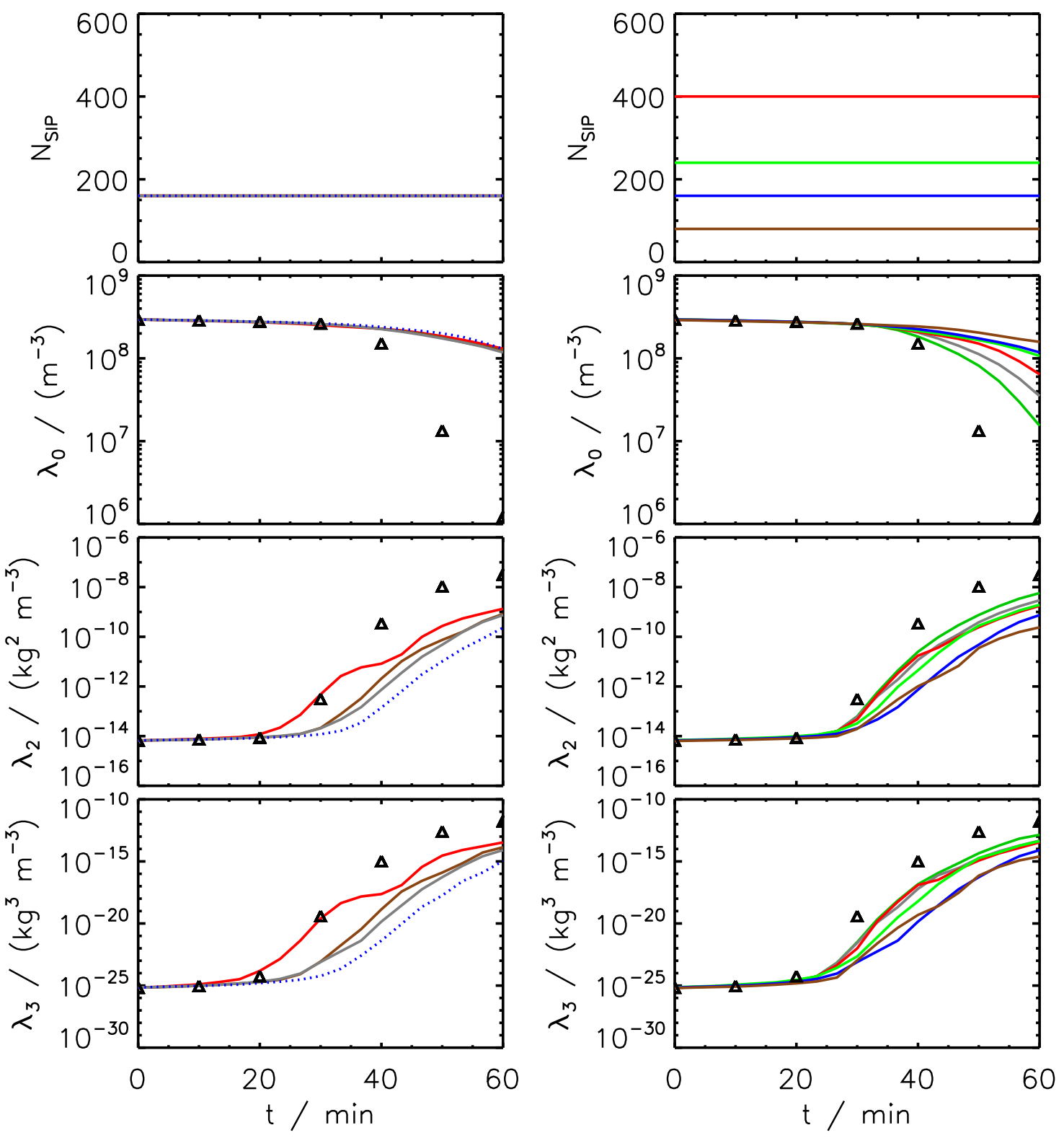

Figure 44: LONG AON: Variation of time step $d t$ and SIP number $N_{S I P}$. 


\subsubsection{Long Kernel (AON), $\nu_{\text {random }, r s}$-init}

\subsubsection{Variation of $d t$ and $\alpha_{\text {low }}$}
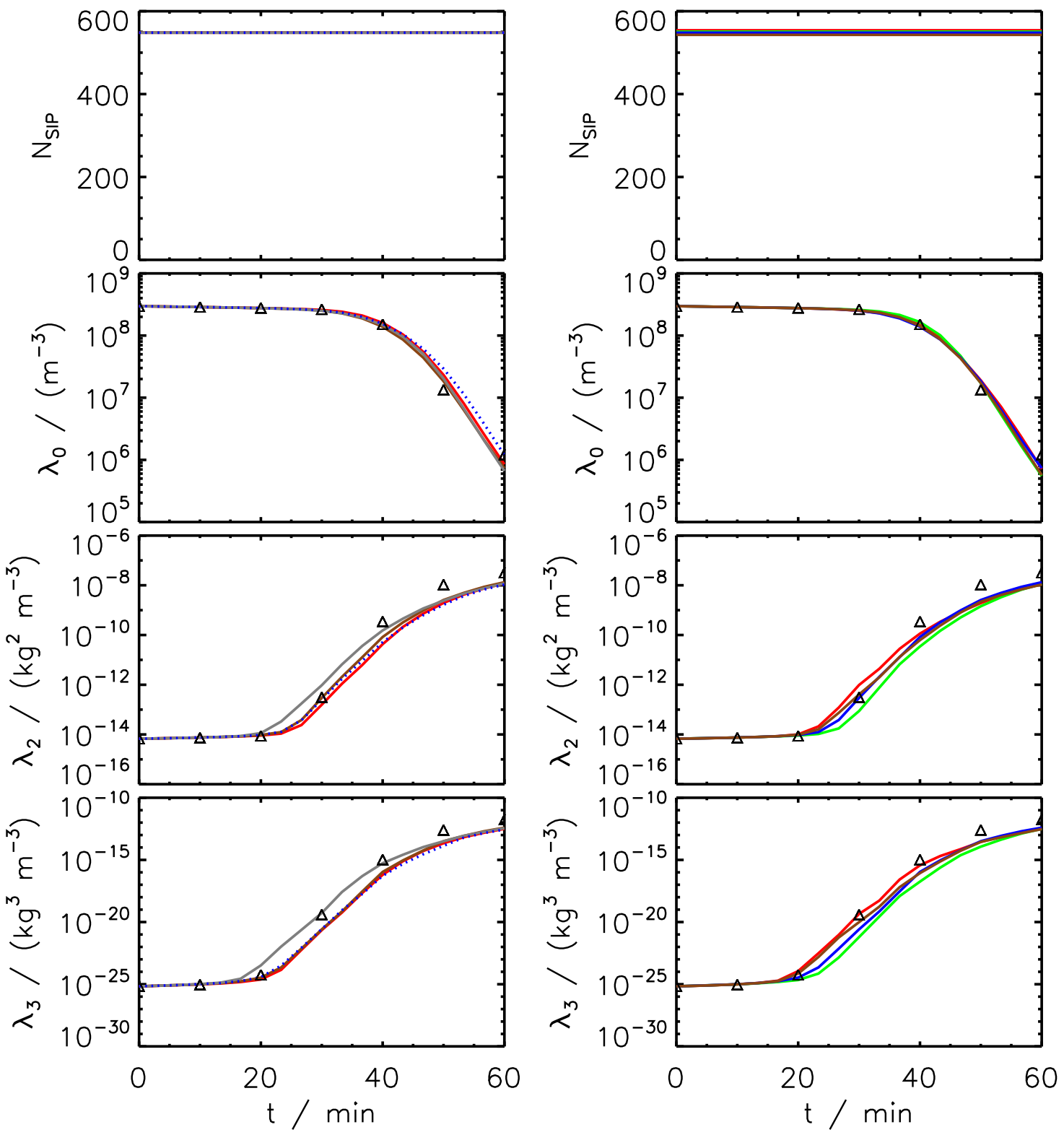

Figure 45: LONG AIM: Variation of time step $d t$ (left) and lower threshold $\alpha_{\text {low }}$ (right, at $d t=5 \mathrm{~s})$. 


\subsubsection{Long Kernel (AON), $\nu_{\text {random }, l b}$-init}

\subsubsection{Variation of $d t$ and $\alpha_{\text {low }}$}
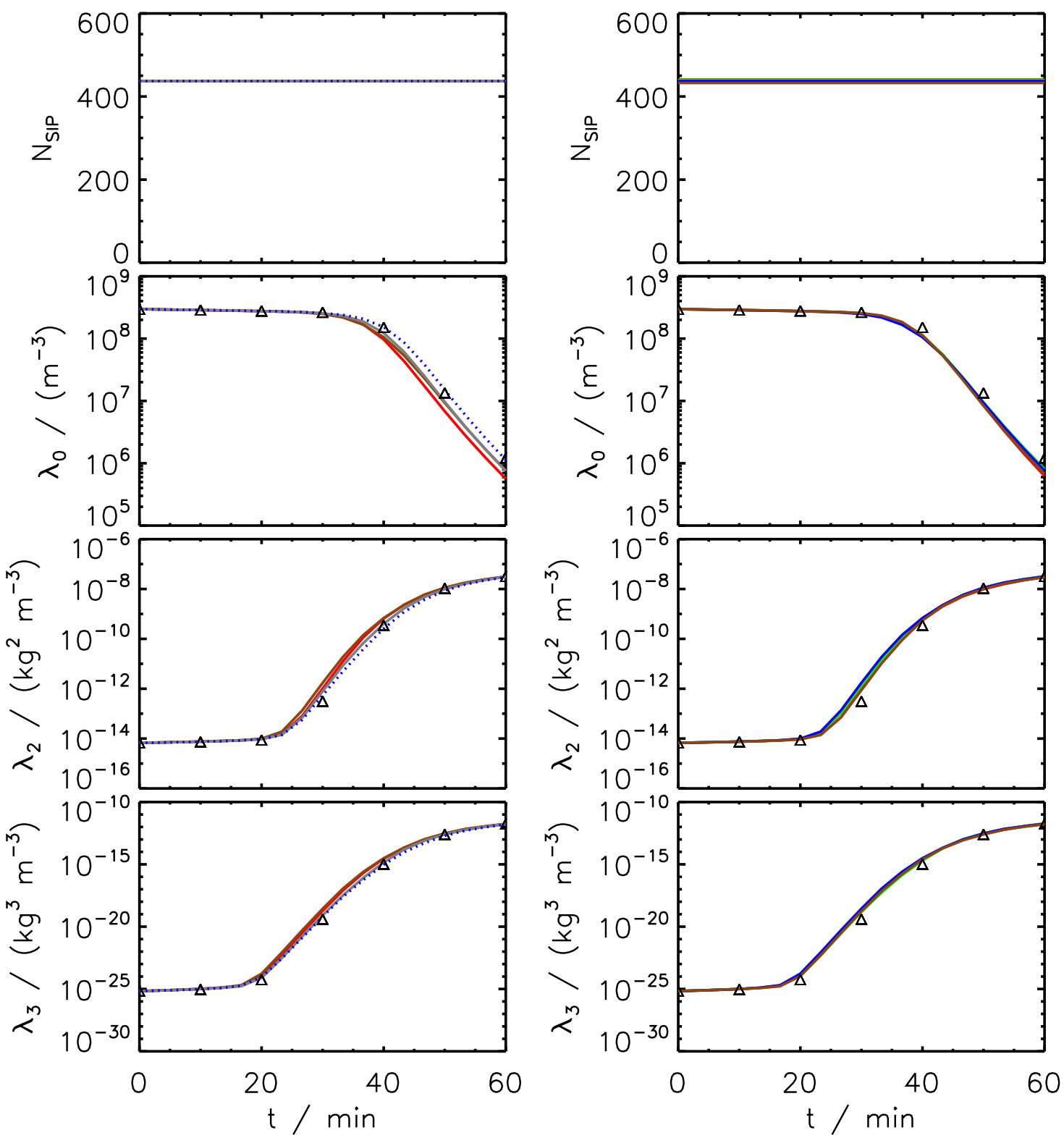

Figure 46: LONG AIM: Variation of time step $d t$ (left) and lower threshold $\alpha_{\text {low }}$ (right, at $d t=5 \mathrm{~s})$. 


\subsection{Hall Kernel (AON)}

The following sections show Long AON results for three types of init methods. 


\subsubsection{Hall Kernel (AON), SingleSIP-init}

\subsubsection{Variation of $d t$ and $\kappa$}
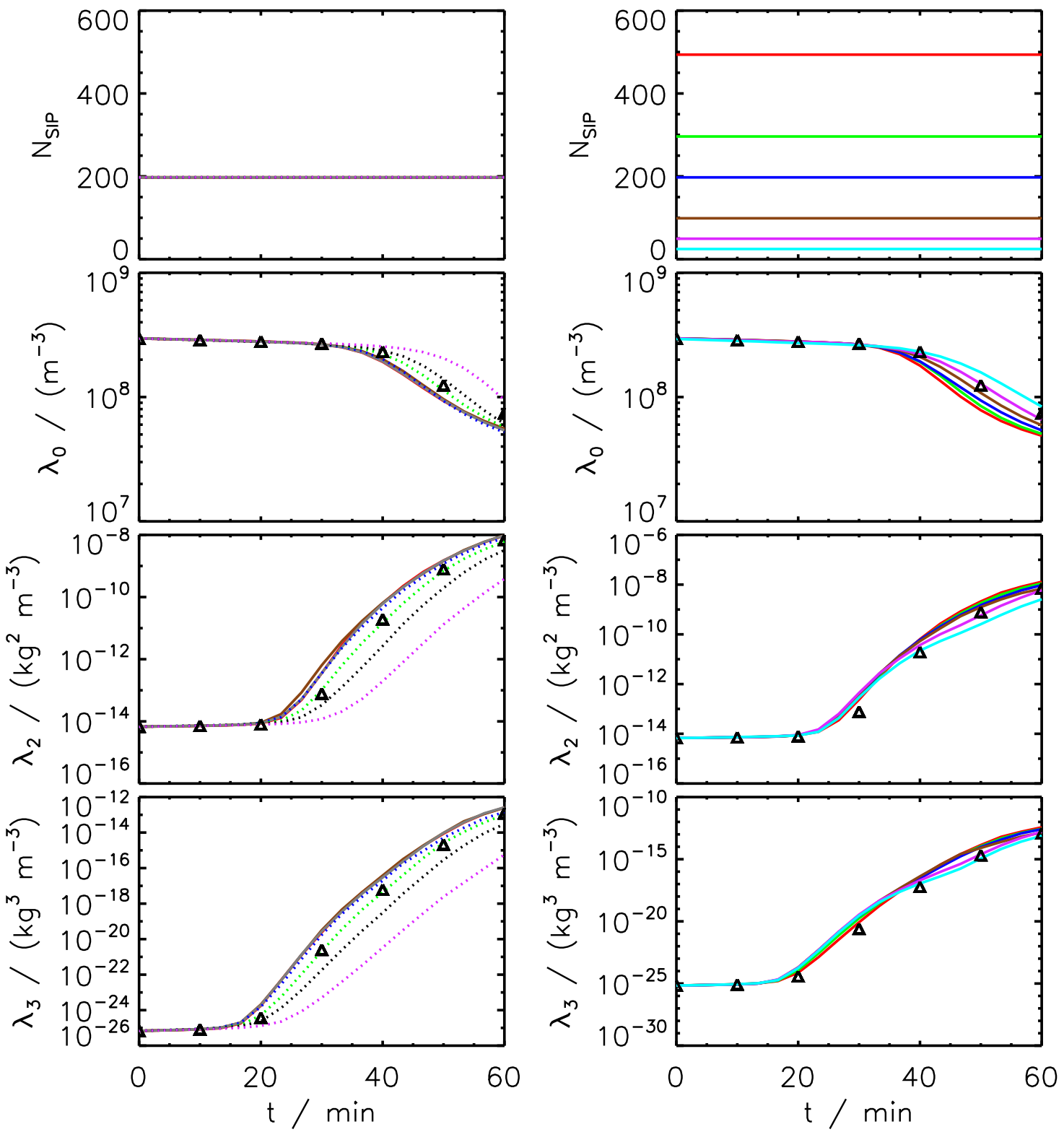

Figure 47: HALL AON: Variation of time step $d t$ (left) and bin resolution $\kappa$ (right). 


\subsubsection{Variation of $\eta$}

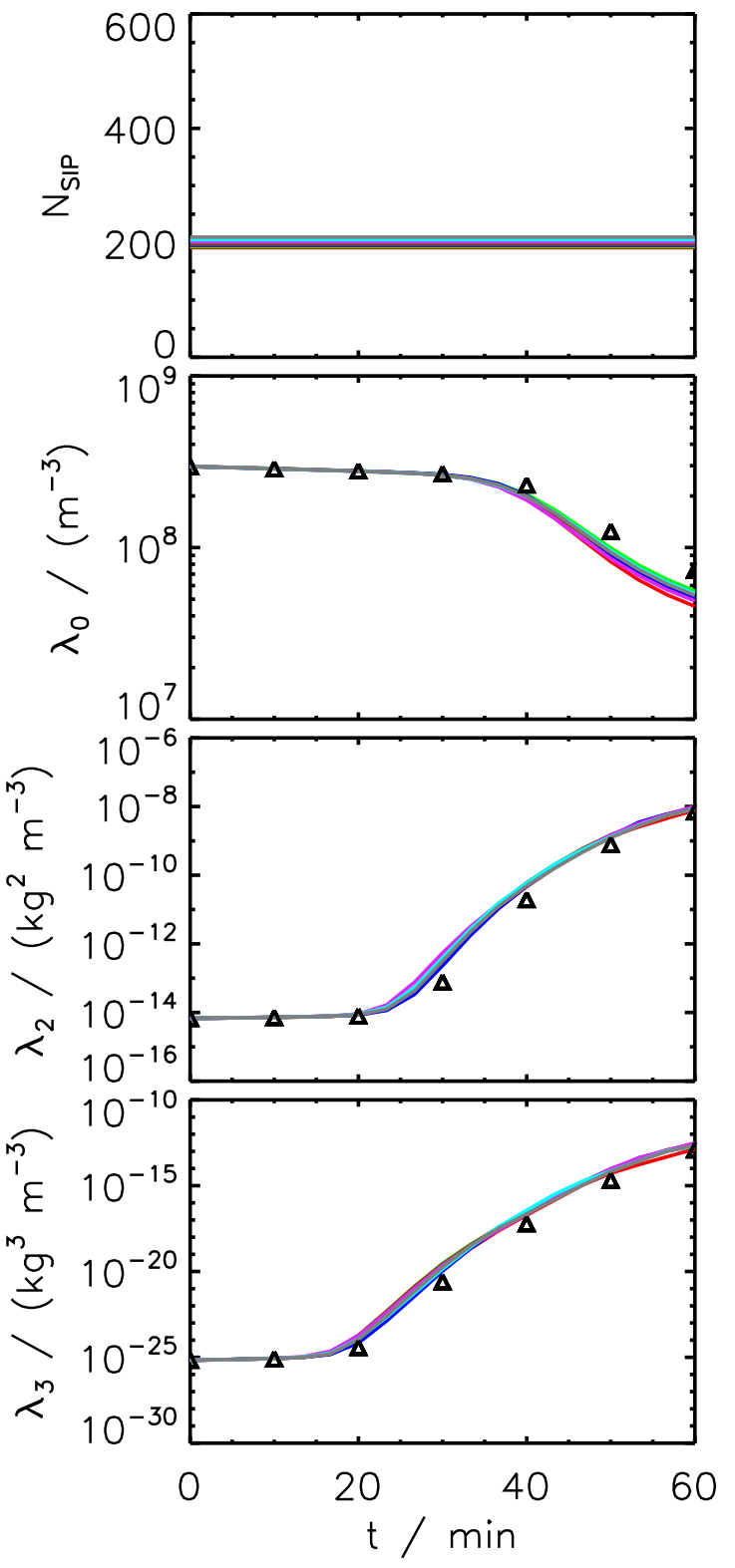

Figure 48: HALL AON: Variation of threshold $\eta$. 


\subsubsection{Variation of $r_{\text {critmin }}$}
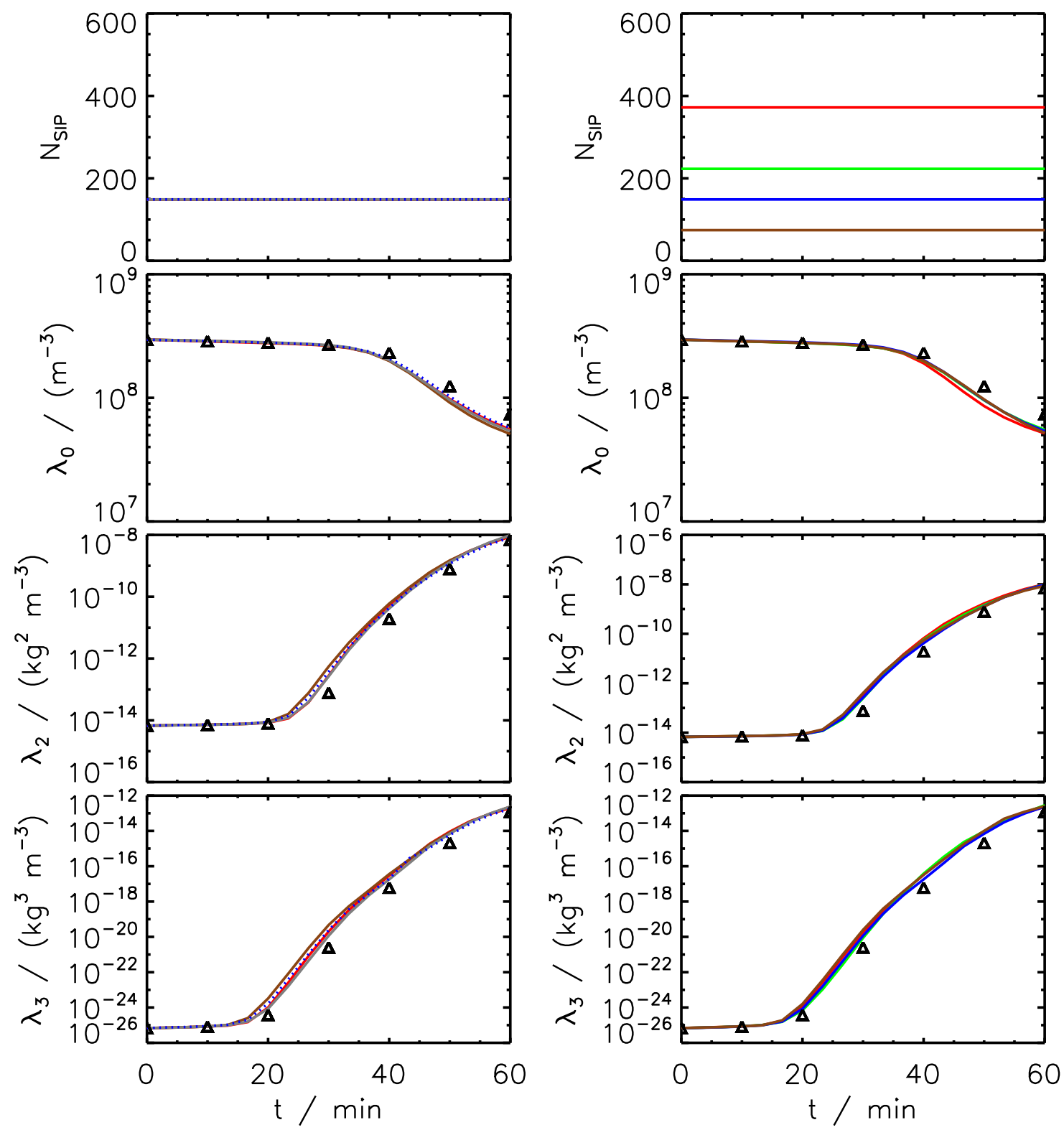

Figure 49: HALL AON: Variation of of time step $d t$ (left) and bin resolution $\kappa$ (right) at $r_{\text {critmin }}=1.6 \mu \mathrm{m}$. 


\subsubsection{Hall Kernel (AON), $\nu_{\text {const }}$-init}

\subsubsection{Variation of $d t$ and $N_{S I P}$}
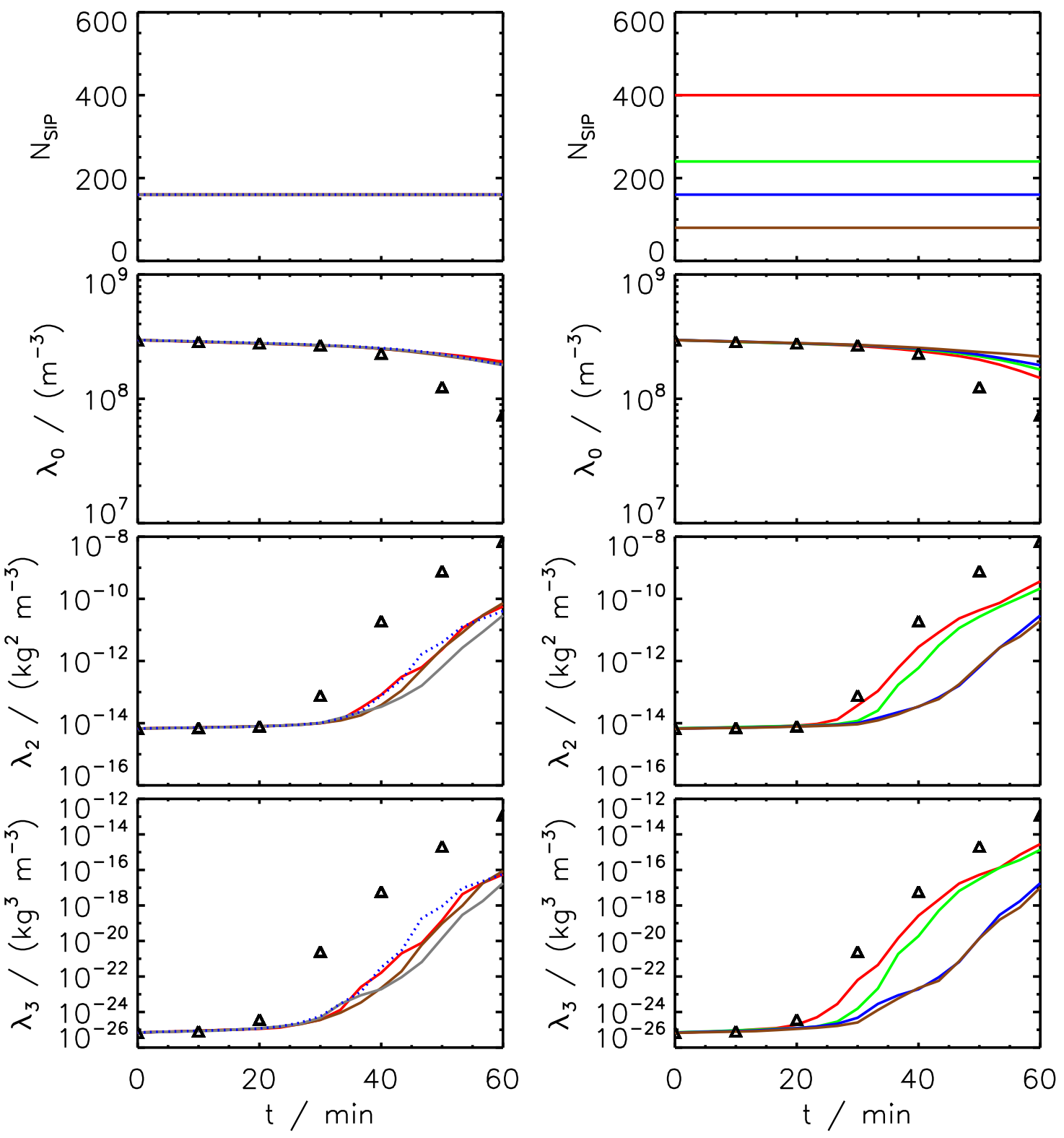

Figure 50: HALL AON: Variation of time step $d t$ and SIP number $N_{S I P}$. 


\subsubsection{Hall Kernel (AON), $\nu_{d r a w}$-init}

\subsubsection{Variation of $d t$ and $N_{S I P}$}
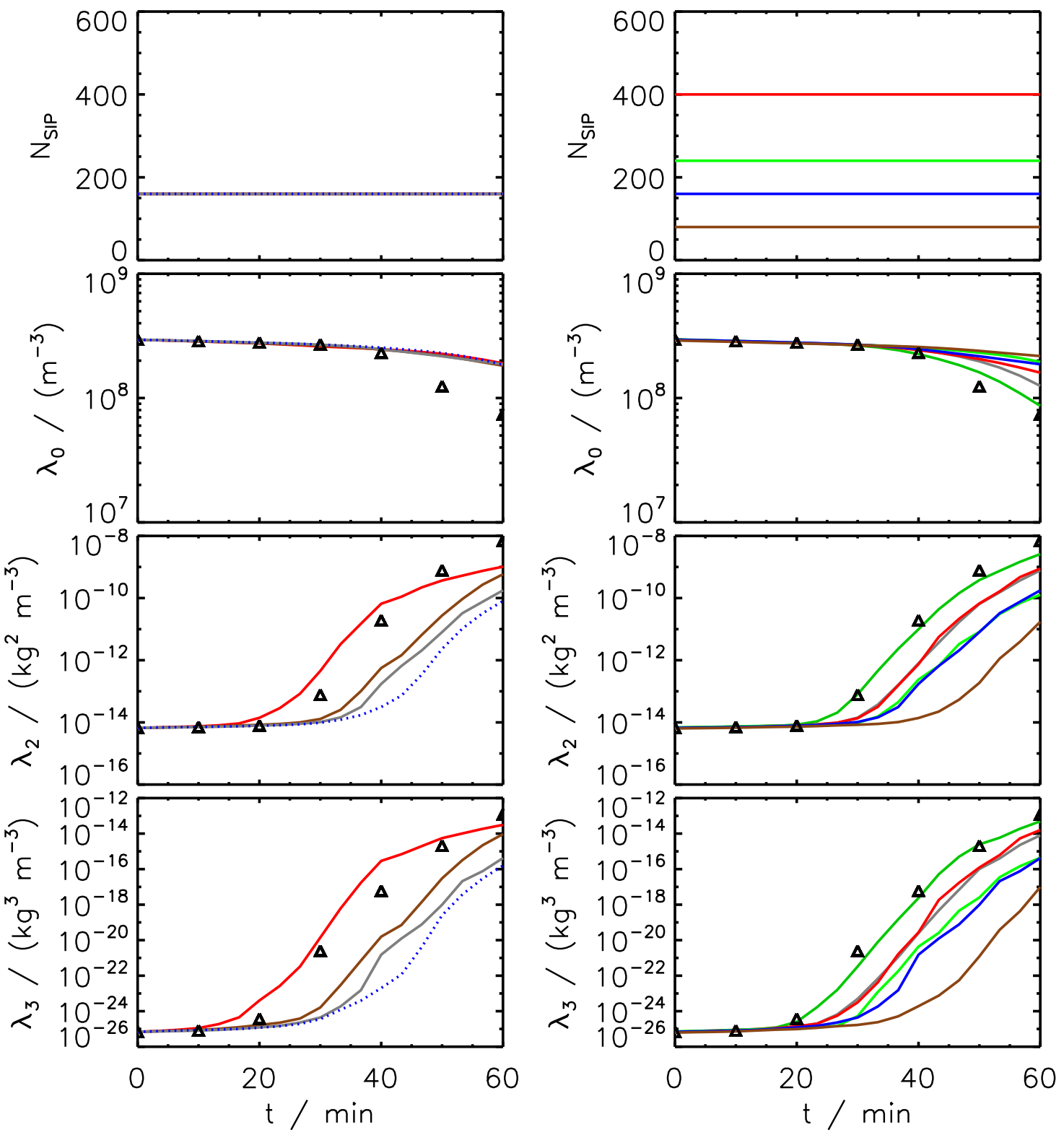

Figure 51: HALL AON: Variation of time step $d t$ and SIP number $N_{S I P}$. 


\subsection{Constant efficiency Kernel (AON)}

This subsection shows simulations for a hydrodynamic kernel with constant efficiency $E_{c}$, which is assigned a value of 1.0 or 0.2 . 


\subsubsection{Collection efficiency $E_{c}=0.2$, SingleSIP-init}

\subsubsection{Variation of $d t$ and $\kappa$}
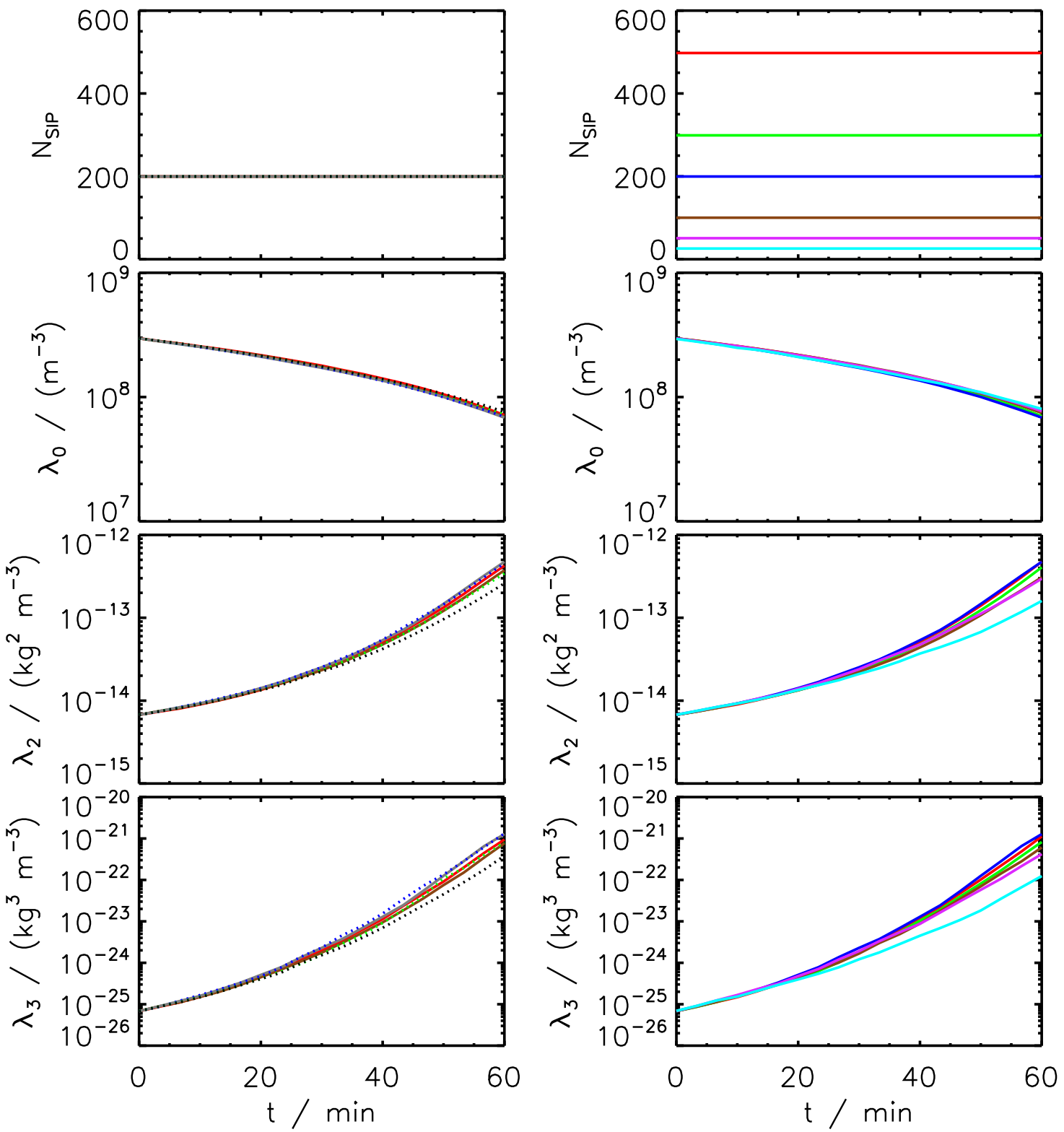

Figure 52: $\mathrm{EC}=0.2 \mathrm{AON}$ : Variation of time step $d t$ (left) and bin resolution $\kappa$ (right). 


\subsubsection{Collection efficiency $E_{c}=1.0$, SingleSIP-init}

\subsubsection{Variation of $\kappa$}

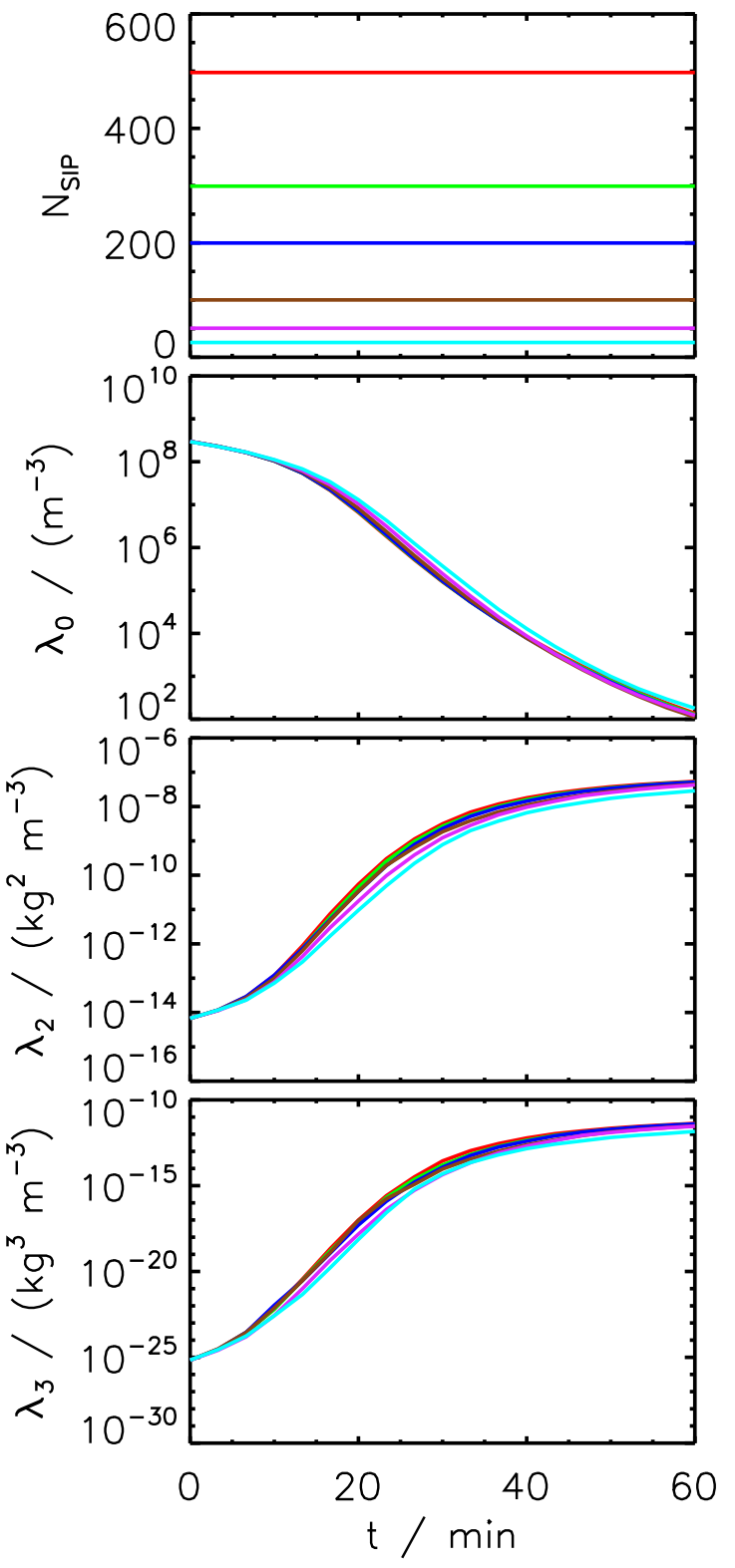

Figure 53: $\mathrm{EC}=1.0 \mathrm{AON}$ : Variation of bin resolution $\kappa$. 